\title{
Synthesis of Enantioenriched $\beta$-Aryl- $\beta$-Aryloxy Esters via Sequential Photoisomerization and Enantioselective Hydrogenation
}

\author{
Elizabeth Swift, * Shashank Shekhar and Brian Kotecki
}

AbbVie, Inc., Process Research and Development, 1 N. Waukegan Road, North Chicago, IL-6oo64, United States

\section{Contents}

1. General Methods

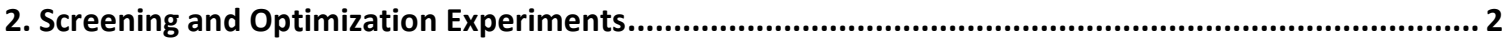

2.1. Screen of Oxa-Michael Reaction Conditions ...................................................................... 2

2.2. Optimization of Conditions for Photoisomerization ........................................................ 3

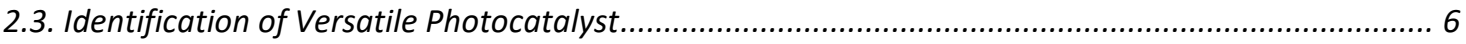

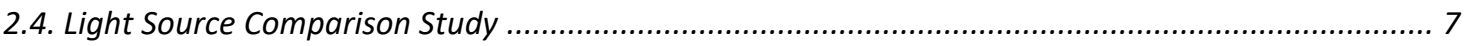

2.5. Asymmetric Hydrogenation Ligand Screen ................................................................. 8

2.6. Solvent Screen for Asymmetric Hydrogenation ................................................................ 12

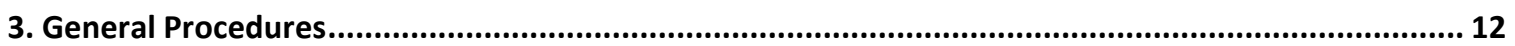

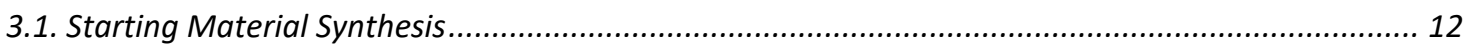

3.2. Oxa-Michael, Isomerization, and Reduction Standard Procedures............................................ 13

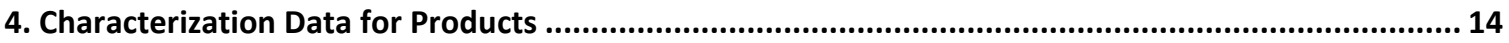

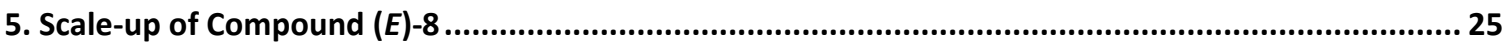

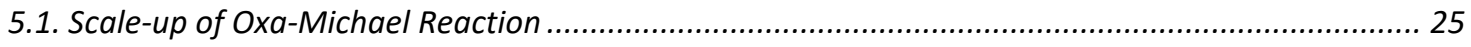

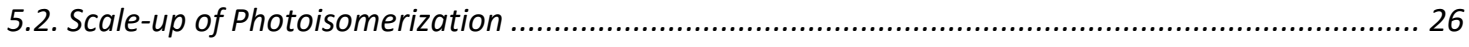

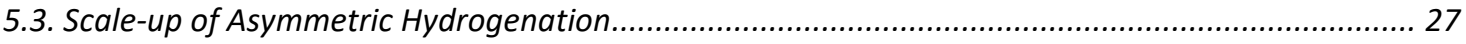

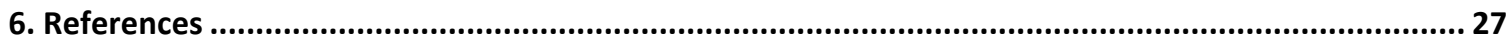

7. Crystallographic Data for 15 Single Crystal X-ray Structure ............................................... 27

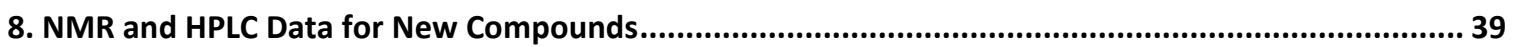




\section{General Methods}

Anhydrous grade solvents, metal complexes, and phosphine ligands were purchased from commercial suppliers and were used without further purification. Column chromatography was performed using pre-packed silica gel columns on a Combi flash companion automated column. ${ }^{1} \mathrm{H}$ NMR and ${ }^{13} \mathrm{C}$ NMR spectra were recorded on a Varian 400 or 600 $\mathrm{MHz}$ spectrometer, with shifts reported in parts per million downfield from tetramethylsilane and referenced to residual proton $\left({ }^{1} \mathrm{H}\right)$ or deuterated solvent $\left({ }^{13} \mathrm{C}\right)$. HPLC analysis were performed on an Agilent 1100 or 1200 series instrument using spectroscopic grades of acetonitrile and water with either $0.1 \mathrm{wt} \% \mathrm{H}_{3} \mathrm{PO}_{4}$ or $0.1 \% \mathrm{HClO}_{4}$ as eluents. Melting point analyses were performed on a Mettler FP62 at a temperature ramp of 1 ${ }^{\circ} \mathrm{C} / \mathrm{min}$ or on a Thomas Hoover Uni-melt. Elemental analyses were performed by QTI (Whitehouse, $\mathrm{NJ}$ ).

Laser Safety Details and Disclaimer: All lasers described in this manuscript are class IV lasers and appropriate safety precautions were taken. Readers are cautioned to follow all government and institutional regulations prior to using lasers as light sources. Properly rated eyewear was always worn. In discussions with AbbVie radiation safety officers two significant observations were made that directly impacted the use of any laser described in this manuscript. First, the lasers made use of optics which expanded the beam by a significant degree. Had any beam been focused, much higher safety precautions would be needed. Second, the beams were always directed into solutions designed to absorb much of, if not of all the light. Lasers were always directed downward into solutions containing photocatalysts. ${ }^{1}$

\section{Screening and Optimization Experiments}

\subsection{Screen of Oxa-Michael Reaction Conditions}

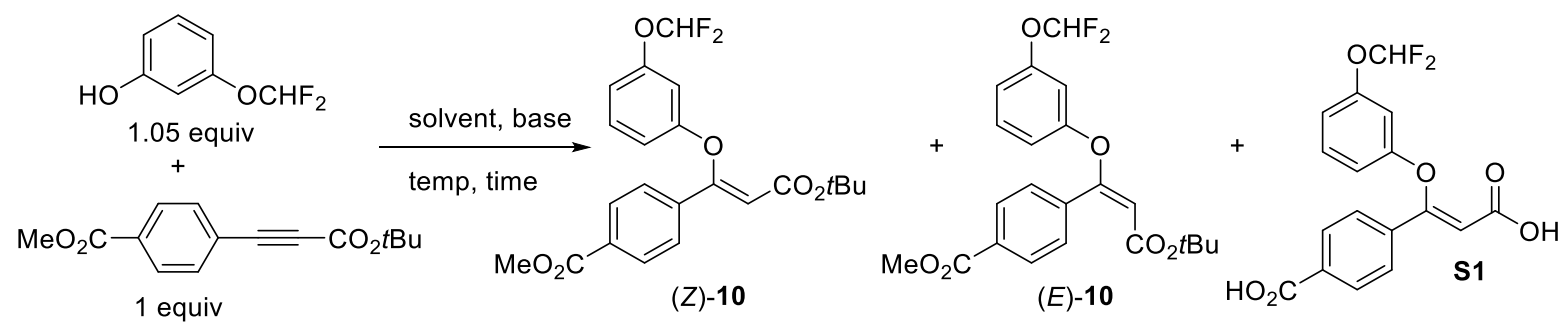

Table S1. Results for oxa-Michael reaction under different conditions

\begin{tabular}{cccccccccc}
\hline Entry & Base (equiv) & Solvent & $\begin{array}{c}\text { Conc } \\
(\mathrm{M})\end{array}$ & $\begin{array}{c}\text { Temp } \\
\left({ }^{\circ} \mathrm{C}\right)\end{array}$ & $\begin{array}{c}\text { Time } \\
(\mathrm{h})\end{array}$ & $\begin{array}{c}\text { Conv } \\
(\%)^{\mathrm{a}}\end{array}$ & $\mathbf{1 0}(\%)^{\mathrm{h}}$ & $\begin{array}{c}\text { Ratio of } \\
\mathrm{Z} / \mathrm{E}\end{array}$ & $\mathbf{S 1}(\%)^{\mathrm{c}}$ \\
\hline 1 & $\mathrm{~K}_{2} \mathrm{CO}_{3}(2.5)$ & DMF & 0.58 & 70 & 0.67 & 100 & 75 & $3: 1^{\mathrm{d}}$ & 17 \\
2 & $\mathrm{~K}_{2} \mathrm{CO}_{3}(1.5)$ & $\mathrm{DMF}$ & 0.38 & 50 & 1.75 & 100 & 79 & $4.5: 1^{\mathrm{d}}$ & 10 \\
3 & $\mathrm{~K}_{2} \mathrm{CO}_{3}(1.0)$ & $\mathrm{DMF}$ & 0.38 & 25 & 20 & 100 & 72 & $2.7: 1^{\mathrm{e}}$ & 15 \\
4 & $\mathrm{~K}_{2} \mathrm{CO}_{3}(1.0)$ & DMF & 0.77 & 25 & 2 & 58 & $35^{\mathrm{i}}$ & $6.4: 1$ & $<0.5$ \\
& & & & & 4.5 & 100 & 86 & $2.7: 1^{\mathrm{e}}$ & 5 \\
5 & $\mathrm{~K}_{3} \mathrm{PO}_{4}(1.0)$ & DMF & 0.77 & 25 & 2 & 100 & 62 & $5.6: 1$ & $23^{\mathrm{g}}$
\end{tabular}




\begin{tabular}{|c|c|c|c|c|c|c|c|c|c|}
\hline Entry & Base (equiv) & Solvent & $\begin{array}{l}\text { Conc } \\
\text { (M) }\end{array}$ & $\begin{array}{l}\text { Temp } \\
\left({ }^{\circ} \mathrm{C}\right)\end{array}$ & $\begin{array}{l}\text { Time } \\
\text { (h) }\end{array}$ & $\begin{array}{l}\text { Conv } \\
(\%)^{\mathrm{a}}\end{array}$ & $10(\%)^{\mathrm{h}}$ & $\begin{array}{c}\text { Ratio of } \\
\text { Z/E }\end{array}$ & $\mathbf{S 1}(\%)^{\mathrm{c}}$ \\
\hline 6 & $\mathrm{Cs}_{2} \mathrm{CO}_{3}(1.0)$ & DMF & 0.77 & 25 & 2 & 100 & 76 & $7: 1$ & $14^{\mathrm{g}}$ \\
\hline \multirow[t]{3}{*}{7} & $\mathrm{~K}_{2} \mathrm{CO}_{3}(1.0)$ & DMF & 0.85 & 25 & 2 & 52 & $21^{\mathrm{i}}$ & $6.1: 1$ & $<0.5$ \\
\hline & & & & & 4 & 99 & 72 & $5.2: 1$ & 23 \\
\hline & & & & 50 & 2 & 1 & NA & NA & $<0.5$ \\
\hline \multirow[t]{3}{*}{8} & $\mathrm{~K}_{2} \mathrm{CO}_{3}(1.5)$ & Toluene & 0.38 & 80 & 2.45 & 31 & $14^{\mathrm{i}}$ & NA & $<0.5$ \\
\hline & & & & & 18.5 & 81 & $67^{\mathrm{i}}$ & $2.7: 1^{\mathrm{e}}$ & 2 \\
\hline & & & & 50 & 2 & 6 & $2.5^{\mathrm{i}}$ & NA & $<0.5$ \\
\hline \multirow[t]{2}{*}{9} & $\mathrm{~K}_{2} \mathrm{CO}_{3}(1.5)$ & THF & 0.38 & 65 & $2.45^{\mathrm{f}}$ & 62 & $42^{\mathrm{i}}$ & NA & $<0.5$ \\
\hline & & & & & $18.5^{\mathrm{f}}$ & 92 & $79^{i}$ & $2.8: 1^{\mathrm{e}}$ & 1 \\
\hline \multirow{2}{*}{10} & $\mathrm{~K}_{2} \mathrm{CO}_{3}(1.5)$ & Dioxane & 0.38 & 50 & 2 & 2 & 1 & NA & NA \\
\hline & & & & 80 & $18.5^{\mathrm{f}}$ & 99 & 87 & $2.8: 1^{\mathrm{e}}$ & 2 \\
\hline \multirow[t]{3}{*}{11} & $\mathrm{~K}_{2} \mathrm{CO}_{3}(1.5)$ & Propylene & 0.38 & 50 & 2 & 28 & $13^{\mathrm{i}}$ & NA & $<0.5$ \\
\hline & & Carbonate & & 65 & $2.45^{\mathrm{f}}$ & 98 & 85 & $2.5: 1$ & $<0.5$ \\
\hline & & & & & 1.75 & 27 & $13^{\mathrm{i}}$ & NA & $<0.5$ \\
\hline \multirow[t]{2}{*}{12} & DABCO (1.0) & $\mathrm{MeCN}$ & 0.38 & 50 & 3.25 & 62 & $36^{\mathrm{i}}$ & NA & $<0.5$ \\
\hline & & & & & 73 & 100 & 75.6 & $2.5: 1^{\mathrm{d}}$ & 12 \\
\hline 13 & $\mathrm{~K}_{2} \mathrm{CO}_{3}(1.0)$ & $\mathrm{MeCN}$ & 0.38 & 50 & 2.6 & 98 & 79 & $4.8: 1^{\mathrm{e}}$ & 13 \\
\hline 14 & $\mathrm{~K}_{2} \mathrm{CO}_{3}(1.0)$ & Acetone & 0.38 & 50 & 18 & 65 & 54 & NA & 0.6 \\
\hline 15 & $\mathrm{~K}_{2} \mathrm{CO}_{3}(1.0)$ & EtOAc & 0.38 & 50 & 27 & 95 & 92 & 3.7 & 0.6 \\
\hline 16 & $\mathrm{~K}_{2} \mathrm{CO}_{3}(1.0)$ & DCE & 0.38 & 50 & 18 & 30 & 21 & NA & 0.1 \\
\hline 17 & $\mathrm{~K}_{2} \mathrm{CO}_{3}(1.0)$ & DMSO & 0.38 & 50 & 1 & 100 & 73 & 6.5 & 13 \\
\hline 18 & $\mathrm{Na}_{2} \mathrm{CO}_{3}(1.0)$ & DMSO & 0.38 & 50 & 21 & 100 & 99 & 5.8 & 0.1 \\
\hline 19 & $\mathrm{~K}_{2} \mathrm{HPO}_{4}(1.0)$ & DMSO & 0.38 & 50 & 21 & 100 & 98 & 6.2 & 1.4 \\
\hline 20 & $\mathrm{Na}_{3} \mathrm{PO}_{4}(1.0)$ & DMSO & 0.51 & 50 & 18 & 99 & 95 & 5.9 & 1.7 \\
\hline 21 & $\mathrm{Na}_{2} \mathrm{HPO}_{4}(1.0)$ & DMSO & 0.51 & 50 & 18 & 2 & 2 & NA & 0 \\
\hline 22 & $\mathrm{Li}_{2} \mathrm{CO}_{3}(1.0)$ & DMSO & 0.51 & 50 & 18 & 70 & 68 & NA & 0 \\
\hline 23 & $\mathrm{Ca}(\mathrm{OH})_{2}(1.0)$ & DMSO & 0.51 & 50 & 18 & 99 & 78 & 6.2 & 15 \\
\hline 24 & KOAc (1.0) & DMSO & 0.51 & 50 & 18 & 99 & 88 & $6.7^{\mathrm{j}}$ & 0.3 \\
\hline 25 & $\mathrm{Na}_{2} \mathrm{CO}_{3}(1.0)$ & DMSO & 1.3 & 50 & 20 & 92 & 91 & 4.9 & 0 \\
\hline 26 & $\mathrm{~K}_{2} \mathrm{HPO}_{4}(1.0)$ & DMSO & 1.3 & 50 & 20 & 97 & 94 & 5.1 & 2.6 \\
\hline 27 & $\mathrm{Na}_{2} \mathrm{CO}_{3}(1.0)$ & DMSO & 0.77 & 50 & 24 & 96 & 94 & 5.6 & 0 \\
\hline 28 & $\mathrm{Na}_{2} \mathrm{CO}_{3}(2.0)$ & DMSO & 0.77 & 50 & 24 & 92 & 92 & 5.5 & 0 \\
\hline 29 & $\mathrm{Na}_{2} \mathrm{CO}_{3}(2.0)$ & DMSO & 0.77 & 35 & 27 & 64 & 63 & 5.8 & 0 \\
\hline 30 & $\mathrm{Na}_{2} \mathrm{CO}_{3}(1.0)$ & DMSO & 0.51 & 50 & 15 & 100 & 98 & 5.8 & 0 \\
\hline
\end{tabular}

(a) 100\% - [100 x (alkyne area\%)/(Z + E + alkyne + S1)area\%] @ $210 \mathrm{~nm}$. (b) 2 equivalents of phenol. (c) (S1 area\% x 100)/(Z + E + alkyne + S1)area\% @ $210 \mathrm{~nm}$. (d) Z/E ratio was determined by ${ }^{1} \mathrm{H}$ NMR. (e) Z/E ratio was determined by chiral HPLC. (f) The $1^{\text {st }} 2$ hours of the reaction was at $50{ }^{\circ} \mathrm{C}$. (g) Several other unidentified impurities were observed. (h) Z+E area\% excluding the solvent peak @ $254 \mathrm{~nm}$. The DP area\% is calculated at $254 \mathrm{~nm}$ because the solvents used in the above set of experiments do not absorb strongly at $254 \mathrm{~nm}$. (i) The low DP area\% is because of the low absorbance of the DP at 254 than the alkyne starting material. (j) Several small peaks were observed in the HPLC spectra that are not present with other bases.

\subsection{Optimization of Conditions for Photoisomerization}

Inside a glovebox, a 96-well plate (Analytical Sales and Services, Part \#96973) was equipped with $1 \mathrm{~mL}$ glass vials with one glass stir bead in each well. Catalysts were dosed into the reaction block as stock solutions in MeCN to target $0.05 \mu \mathrm{mol}$ of catalyst per well. The solvent was removed with a stream of nitrogen and then the substrate was dosed as stock solution $(0.025 \mathrm{M})$ in each of the eight solvents $\left(\mathrm{MeCN} / \mathrm{H}_{2} \mathrm{O}\right.$ was a $10: 1$ mixture) to target $5 \mu \mathrm{mol}(200 \mu \mathrm{L})$ of substrate per well. Substrate dosed as a mixture of $37.9 \% E-1$ and $62.1 \% \mathrm{Z}-1$. The reaction block was sealed and placed on a blue LED array light source 
(Analytical Sales and Services, Part \# LUM96B and Part\# LUMCON, set to max power). Blue LEDs were chosen as the light source as they are the most common and readily available visible light source that also match well with the maximum absorbance of all the catalysts tested. After $21 \mathrm{~h}$ stirring on an orbital agitator, a $5 \mu \mathrm{L}$ aliquot of each reaction was diluted to $0.5 \mathrm{~mL}$ with $\mathrm{MeCN}$ for HPLC analysis.
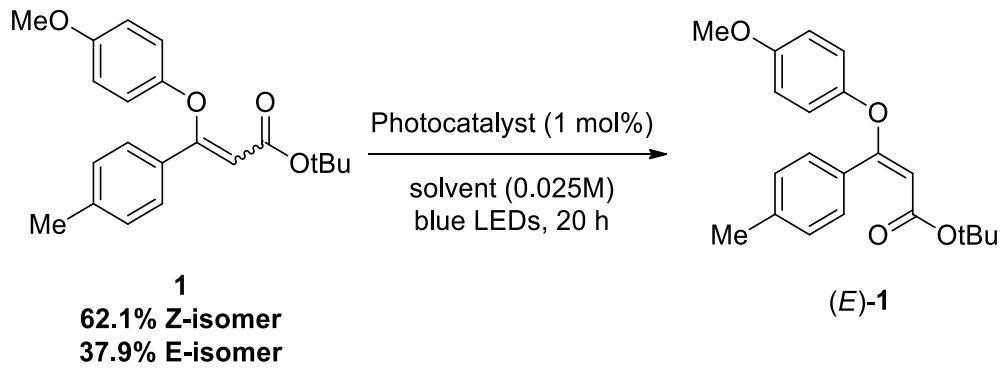

$(E)-\mathbf{1}$

Table S2. Screening results for photoisomerization reaction.

\begin{tabular}{|c|c|c|c|c|c|c|c|}
\hline Entry & Catalyst & Solvent & $\% \mathrm{E}$ & Entry & Catalyst & Solvent & $\% \mathrm{E}$ \\
\hline 1 & {$\left[\mathrm{Ru}(\mathrm{bpy})_{3}\right] \mathrm{Cl}_{2}$} & $\mathrm{MeCN}$ & 39.4 & 49 & $\operatorname{Ir}(\mathrm{dFppy})_{3}$ & $\mathrm{MeCN}$ & 93.2 \\
\hline 2 & {$\left[\mathrm{Ru}(\mathrm{bpy})_{3}\right] \mathrm{Cl}_{2}$} & DMSO & 41.6 & 50 & $\operatorname{Ir}(\mathrm{dFppy})_{3}$ & DMSO & 89.4 \\
\hline 3 & {$\left[\mathrm{Ru}(\mathrm{bpy})_{3}\right] \mathrm{Cl}_{2}$} & $\mathrm{DMF}$ & 38.1 & 51 & $\operatorname{Ir}(\mathrm{dFppy})_{3}$ & DMF & 91.8 \\
\hline 4 & {$\left[\mathrm{Ru}(\mathrm{bpy})_{3}\right] \mathrm{Cl}_{2}$} & DCE & 37.8 & 52 & $\operatorname{Ir}(\mathrm{dFppy})_{3}$ & DCE & 92.0 \\
\hline 5 & {$\left[\mathrm{Ru}(\mathrm{bpy})_{3}\right] \mathrm{Cl}_{2}$} & dioxane & 39.2 & 53 & $\operatorname{Ir}(\text { dFppy })_{3}$ & dioxane & 91.3 \\
\hline 6 & {$\left[\mathrm{Ru}(\mathrm{bpy})_{3}\right] \mathrm{Cl}_{2}$} & toluene & 38.7 & 54 & $\operatorname{Ir}(\mathrm{dFppy})_{3}$ & toluene & 91.0 \\
\hline 7 & {$\left[\mathrm{Ru}(\mathrm{bpy})_{3}\right] \mathrm{Cl}_{2}$} & $\mathrm{MeCN} / \mathrm{H}_{2} \mathrm{O}$ & 39.8 & 55 & $\operatorname{Ir}(\mathrm{dFppy})_{3}$ & $\mathrm{MeCN} / \mathrm{H}_{2} \mathrm{O}$ & 93.0 \\
\hline 8 & {$\left[\mathrm{Ru}(\mathrm{bpy})_{3}\right] \mathrm{Cl}_{2}$} & acetone & 37.7 & 56 & $\operatorname{Ir}(\mathrm{dFppy})_{3}$ & acetone & 97.8 \\
\hline 9 & {$\left[\mathrm{Ru}(\text { phen })_{3}\right] \mathrm{Cl}_{2}$} & $\mathrm{MeCN}$ & 44.1 & 57 & {$\left[\operatorname{Ir}\left(\mathrm{dF}\left(\mathrm{CF}_{3}\right) \mathrm{ppy}\right)_{2}(\mathrm{dtbbpy})\right] \mathrm{PF}_{6}$} & $\mathrm{MeCN}$ & 90.4 \\
\hline 10 & {$\left[\mathrm{Ru}(\text { phen })_{3}\right] \mathrm{Cl}_{2}$} & DMSO & 46.5 & 58 & {$\left[\operatorname{Ir}\left(\mathrm{dF}\left(\mathrm{CF}_{3}\right) \text { ppy }\right)_{2}(\mathrm{dtbbpy})\right] \mathrm{PF}_{6}$} & DMSO & 84.9 \\
\hline 11 & {$\left[\mathrm{Ru}(\text { phen })_{3}\right] \mathrm{Cl}_{2}$} & $\mathrm{DMF}$ & 39.6 & 59 & {$\left[\operatorname{Ir}\left(\mathrm{dF}\left(\mathrm{CF}_{3}\right) \mathrm{ppy}\right)_{2}(\mathrm{dtbbpy})\right] \mathrm{PF}_{6}$} & DMF & 89.6 \\
\hline 12 & {$\left[\mathrm{Ru}(\text { phen })_{3}\right] \mathrm{Cl}_{2}$} & DCE & 37.9 & 60 & {$\left[\operatorname{Ir}\left(\mathrm{dF}\left(\mathrm{CF}_{3}\right) \mathrm{ppy}\right)_{2}(\mathrm{dtbbpy})\right] \mathrm{PF}_{6}$} & DCE & 86.9 \\
\hline 13 & {$\left[\mathrm{Ru}(\text { phen })_{3}\right] \mathrm{Cl}_{2}$} & dioxane & 39.2 & 61 & {$\left[\operatorname{Ir}\left(\mathrm{dF}\left(\mathrm{CF}_{3}\right) \mathrm{ppy}\right)_{2}(\mathrm{dtbbpy})\right] \mathrm{PF}_{6}$} & dioxane & 86.6 \\
\hline 14 & {$\left[\mathrm{Ru}(\text { phen })_{3}\right] \mathrm{Cl}_{2}$} & toluene & 38.9 & 62 & {$\left[\operatorname{Ir}\left(\mathrm{dF}\left(\mathrm{CF}_{3}\right) \mathrm{ppy}\right)_{2}(\mathrm{dtbbpy})\right] \mathrm{PF}_{6}$} & toluene & 86.1 \\
\hline 15 & {$\left[\mathrm{Ru}(\text { phen })_{3}\right] \mathrm{Cl}_{2}$} & $\mathrm{MeCN} / \mathrm{H}_{2} \mathrm{O}$ & 52.7 & 63 & {$\left[\operatorname{Ir}\left(\mathrm{dF}\left(\mathrm{CF}_{3}\right) \mathrm{ppy}\right)_{2}(\mathrm{dtbbpy})\right] \mathrm{PF}_{6}$} & $\mathrm{MeCN} / \mathrm{H}_{2} \mathrm{O}$ & 89.3 \\
\hline 16 & {$\left[\mathrm{Ru}(\text { phen })_{3}\right] \mathrm{Cl}_{2}$} & acetone & 37.7 & 64 & {$\left[\operatorname{Ir}\left(\mathrm{dF}\left(\mathrm{CF}_{3}\right) \mathrm{ppy}\right)_{2}(\mathrm{dtbbpy})\right] \mathrm{PF}_{6}$} & acetone & 90.9 \\
\hline 17 & {$\left[\mathrm{Ru}(\mathrm{dpp})_{3}\right] \mathrm{Cl}_{2}$} & $\mathrm{MeCN}$ & 55.7 & 65 & $\left.[\operatorname{Irppy})_{2}(\mathrm{dtbbpy})\right] \mathrm{PF}_{6}$ & $\mathrm{MeCN}$ & 93.5 \\
\hline 18 & {$\left[\mathrm{Ru}(\mathrm{dpp})_{3}\right] \mathrm{Cl}_{2}$} & DMSO & 45.4 & 66 & {$[\text { Irppy })_{2}($ dtbbpy) $] \mathrm{PF}_{6}$} & DMSO & 87.4 \\
\hline 19 & {$\left[\mathrm{Ru}(\mathrm{dpp})_{3}\right] \mathrm{Cl}_{2}$} & DMF & 40.6 & 67 & $\left.[\operatorname{Irppy})_{2}(\mathrm{dtbbpy})\right] \mathrm{PF}_{6}$ & DMF & 82.3 \\
\hline 20 & {$\left[\mathrm{Ru}(\mathrm{dpp})_{3}\right] \mathrm{Cl}_{2}$} & DCE & 38.9 & 68 & $\left.[\operatorname{Irppy})_{2}(\mathrm{dtbbpy})\right] \mathrm{PF}_{6}$ & DCE & 93.2 \\
\hline 21 & {$\left[\mathrm{Ru}(\mathrm{dpp})_{3}\right] \mathrm{Cl}_{2}$} & dioxane & 39.4 & 69 & $\left.[\text { Irppy })_{2}(\mathrm{dtbbpy})\right] \mathrm{PF}_{6}$ & dioxane & 94.7 \\
\hline 22 & {$\left[\mathrm{Ru}(\mathrm{dpp})_{3}\right] \mathrm{Cl}_{2}$} & toluene & 39.2 & 70 & $\left.[\text { Irppy })_{2}(\mathrm{dtbbpy})\right] \mathrm{PF}_{6}$ & toluene & 93.1 \\
\hline 23 & {$\left[\mathrm{Ru}(\mathrm{dpp})_{3}\right] \mathrm{Cl}_{2}$} & $\mathrm{MeCN} / \mathrm{H}_{2} \mathrm{O}$ & 67.2 & 71 & $\left.[\operatorname{Irppy})_{2}(\mathrm{dtbbpy})\right] \mathrm{PF}_{6}$ & $\mathrm{MeCN} / \mathrm{H}_{2} \mathrm{O}$ & 94.0 \\
\hline 24 & {$\left[\mathrm{Ru}(\mathrm{dpp})_{3}\right] \mathrm{Cl}_{2}$} & acetone & 38.0 & 72 & $\left.[\operatorname{Irppy})_{2}(\mathrm{dtbbpy})\right] \mathrm{PF}_{6}$ & acetone & 92.9 \\
\hline 25 & {$\left[\mathrm{Ru}(\mathrm{bpm})_{3}\right]\left(\mathrm{PF}_{6}\right)_{2}$} & $\mathrm{MeCN}$ & 39.3 & 73 & $\operatorname{Ir}(\text { piq) })_{3}$ & $\mathrm{MeCN}$ & 39.4 \\
\hline 26 & {$\left[\mathrm{Ru}(\mathrm{bpm})_{3}\right]\left(\mathrm{PF}_{6}\right)_{2}$} & DMSO & 41.2 & 74 & $\operatorname{Ir}(\text { piq })_{3}$ & DMSO & 42.2 \\
\hline 27 & {$\left[\mathrm{Ru}(\mathrm{bpm})_{3}\right]\left(\mathrm{PF}_{6}\right)_{2}$} & DMF & 38.0 & 75 & $\operatorname{Ir}(\text { piq })_{3}$ & DMF & 39.0 \\
\hline 28 & {$\left[\mathrm{Ru}(\mathrm{bpm})_{3}\right]\left(\mathrm{PF}_{6}\right)_{2}$} & DCE & 37.9 & 76 & $\operatorname{Ir}(\text { piq })_{3}$ & $\mathrm{DCE}$ & 39.0 \\
\hline 29 & {$\left[\mathrm{Ru}(\mathrm{bpm})_{3}\right]\left(\mathrm{PF}_{6}\right)_{2}$} & dioxane & 39.3 & 77 & $\operatorname{Ir}(\text { piq })_{3}$ & dioxane & 40.6 \\
\hline 30 & {$\left[\mathrm{Ru}(\mathrm{bpm})_{3}\right]\left(\mathrm{PF}_{6}\right)_{2}$} & toluene & 38.8 & 78 & $\operatorname{Ir}(\text { piq })_{3}$ & toluene & 39.3 \\
\hline 31 & {$\left[\mathrm{Ru}(\mathrm{bpm})_{3}\right]\left(\mathrm{PF}_{6}\right)_{2}$} & $\mathrm{MeCN} / \mathrm{H}_{2} \mathrm{O}$ & 38.3 & 79 & $\operatorname{Ir}(\text { piq })_{3}$ & $\mathrm{MeCN} / \mathrm{H}_{2} \mathrm{O}$ & 38.3 \\
\hline 32 & {$\left[\mathrm{Ru}(\mathrm{bpm})_{3}\right]\left(\mathrm{PF}_{6}\right)_{2}$} & acetone & 37.6 & 80 & $\operatorname{Ir}(\text { piq })_{3}$ & acetone & 38.2 \\
\hline 33 & {$\left[\mathrm{Ru}(\mathrm{bpz})_{3}\right]\left(\mathrm{PF}_{6}\right)_{2}$} & $\mathrm{MeCN}$ & 39.3 & 81 & (-)-riboflavin & $\mathrm{MeCN}$ & 41.3 \\
\hline 34 & {$\left[\mathrm{Ru}(\mathrm{bpz})_{3}\right]\left(\mathrm{PF}_{6}\right)_{2}$} & DMSO & 41.3 & 82 & $(-)$-riboflavin & DMSO & 43.4 \\
\hline 35 & {$\left[\mathrm{Ru}(\mathrm{bpz})_{3}\right]\left(\mathrm{PF}_{6}\right)_{2}$} & $\mathrm{DMF}$ & 38.1 & 83 & $(-)$-riboflavin & $\mathrm{DMF}$ & 41.3 \\
\hline
\end{tabular}




\begin{tabular}{|c|c|c|c|c|c|c|c|}
\hline Entry & Catalyst & Solvent & $\% \mathrm{E}$ & Entry & Catalyst & Solvent & $\% \mathrm{E}$ \\
\hline 36 & {$\left[\mathrm{Ru}(\mathrm{bpz})_{3}\right]\left(\mathrm{PF}_{6}\right)_{2}$} & DCE & 37.9 & 84 & (-)-riboflavin & DCE & 38.1 \\
\hline 37 & {$\left[\mathrm{Ru}(\mathrm{bpz})_{3}\right]\left(\mathrm{PF}_{6}\right)_{2}$} & dioxane & 39.1 & 85 & (-)-riboflavin & dioxane & 39.8 \\
\hline 38 & {$\left[\mathrm{Ru}(\mathrm{bpz})_{3}\right]\left(\mathrm{PF}_{6}\right)_{2}$} & toluene & 38.8 & 86 & (-)-riboflavin & toluene & 38.8 \\
\hline 39 & {$\left[\mathrm{Ru}(\mathrm{bpz})_{3}\right]\left(\mathrm{PF}_{6}\right)_{2}$} & $\mathrm{MeCN} / \mathrm{H}_{2} \mathrm{O}$ & 38.2 & 87 & (-)-riboflavin & $\mathrm{MeCN} / \mathrm{H}_{2} \mathrm{O}$ & 42.4 \\
\hline 40 & {$\left[\mathrm{Ru}(\mathrm{bpz})_{3}\right]\left(\mathrm{PF}_{6}\right)_{2}$} & acetone & 38.4 & 88 & $(-)$-riboflavin & acetone & 38.6 \\
\hline 41 & $\operatorname{Ir}(\text { ppy })_{3}$ & $\mathrm{MeCN}$ & 94.9 & 89 & Mes-acridiuium & $\mathrm{MeCN}$ & 39.2 \\
\hline 42 & $\operatorname{Ir}(\mathrm{ppy})_{3}$ & DMSO & 95.4 & 90 & Mes-acridiuium & DMSO & 41.3 \\
\hline 43 & $\operatorname{Ir}(\mathrm{ppy})_{3}$ & DMF & 94.0 & 91 & Mes-acridiuium & DMF & 38.1 \\
\hline 44 & $\operatorname{Ir}(\mathrm{ppy})_{3}$ & DCE & 96.5 & 92 & Mes-acridiuium & DCE & 38.0 \\
\hline 45 & $\operatorname{Ir}(\mathrm{ppy})_{3}$ & dioxane & 95.7 & 93 & Mes-acridiuium & dioxane & 39.1 \\
\hline 46 & $\operatorname{Ir}(\mathrm{ppy})_{3}$ & toluene & 92.3 & 94 & Mes-acridiuium & toluene & 38.9 \\
\hline 47 & $\operatorname{Ir}(\mathrm{ppy})_{3}$ & $\mathrm{MeCN} / \mathrm{H}_{2} \mathrm{O}$ & 96.1 & 95 & Mes-acridiuium & $\mathrm{MeCN} / \mathrm{H}_{2} \mathrm{O}$ & 38.3 \\
\hline 48 & $\operatorname{Ir}(\mathrm{ppy})_{3}$ & acetone & 96.3 & 96 & Mes-acridiuium & acetone & 37.7 \\
\hline
\end{tabular}

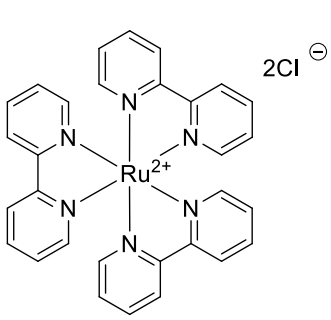

$\left[\mathrm{Ru}(\mathrm{bpy})_{3}\right] \mathrm{Cl}_{2}$

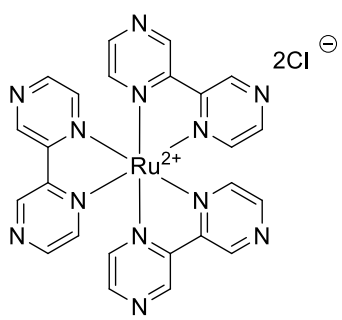

$\left[\mathrm{Ru}(\mathrm{bpz})_{3}\right] \mathrm{Cl}_{2}$ $\mathrm{E}_{\mathrm{T}}=46.5 \mathrm{kcal} / \mathrm{mol}^{2 \mathrm{a}}$

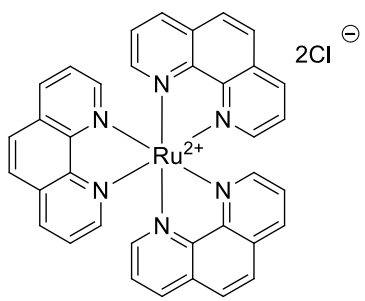

$\left[\mathrm{Ru}(\text { phen })_{3}\right] \mathrm{Cl}_{2}$<smiles></smiles>

$\operatorname{Ir}(\text { ppy })_{3}$ $\mathrm{E}_{\mathrm{T}}=55.2 \mathrm{kcal} / \mathrm{mol}^{2 \mathrm{~b}}$

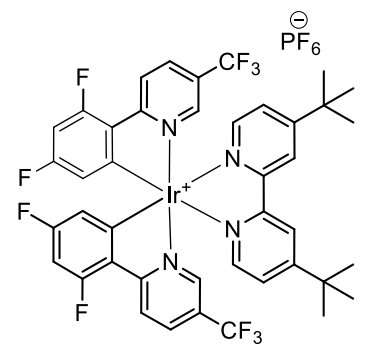

$\left[\operatorname{Ir}\left(\mathrm{dF}\left(\mathrm{CF}_{3}\right) \mathrm{ppy}\right)_{2}(\mathrm{dtbbpy})\right] \mathrm{PF}_{6}$ $E_{T}=60.1 \mathrm{kcal} / \mathrm{mol}^{2 \mathrm{~b}}$

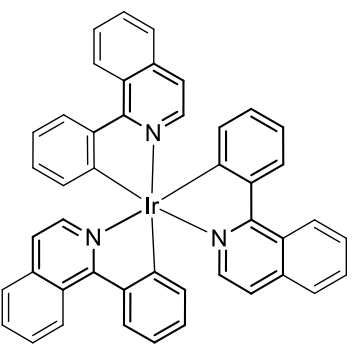

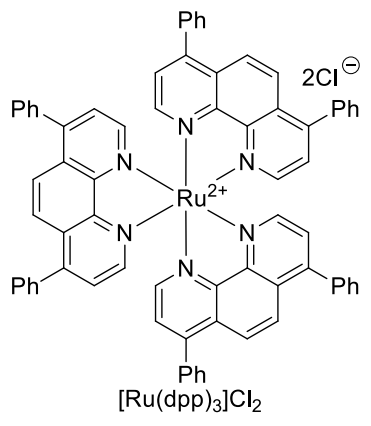

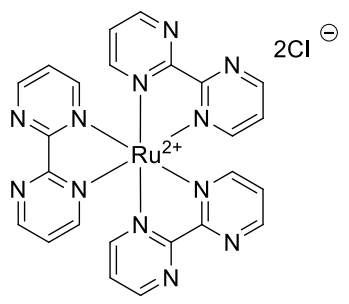

$\left[\mathrm{Ru}(\mathrm{bpm})_{3}\right] \mathrm{Cl}_{2}$<smiles></smiles><smiles></smiles>

$\left[\operatorname{lr}(\text { ppy })_{2}\right.$ (dtbbpy) $] \mathrm{PF}_{6}$ $\mathrm{E}_{\mathrm{T}}=49.2 \mathrm{kcal} / \mathrm{mol}^{2 \mathrm{a}}$<smiles>Cc1cc(C)c(-c2c3ccccc3[n+](C)c3ccccc23)c(C)c1</smiles>

$(-)$-riboflavin
$E_{T}=50.0 \mathrm{kcal} / \mathrm{mol}^{2 \mathrm{c}}$<smiles>Cc1cc2nc3c(=O)[nH]c(=O)nc-3n(C[C@H](O)[C@H](O)[C@H](O)CO)c2cc1C</smiles>

Mes-acridinium $\mathrm{E}_{\mathrm{T}}=54.7 \mathrm{kcal} / \mathrm{mol}^{2 \mathrm{c}}$

Figure S1. Structures of photocatalysts used in photoisomerization screen and selected triplet state energies. ${ }^{2}$ 


\subsection{Identification of Versatile Photocatalyst}

Inside a glovebox, a 24-well plate (Analytical Sales and Services, Part \#24253) was equipped with $1 \mathrm{~mL}$ glass vials with one glass stir bead in each well. Catalysts were dosed into the reaction block as stock solutions in $\mathrm{MeCN}$ to target $0.05 \mu \mathrm{mol}$ of catalyst per well. The solvent was removed with a stream of nitrogen and then the substrates were dosed as stock solutions $(0.025 \mathrm{M})$ in DMSO to target $5 \mu \mathrm{mol}(200 \mu \mathrm{L})$ of substrate per well. The reaction block was sealed and placed on a blue LED array light source (Analytical Sales and Services, Part \# LUM24B and Part\# LUMCON, set to max power). After $20 \mathrm{~h}$ stirring on an orbital agitator, a $5 \mu \mathrm{L}$ aliquot of each reaction was diluted to $0.5 \mathrm{~mL}$ with $\mathrm{MeCN}$ for HPLC analysis.

Note: Since solvent did not have a significant impact on photoisomerization and DMSO was the optimal solvent for the preceding oxa-Michael addition reaction (Table S1), it was chosen as solvent for this study.<smiles>CCCCOC(=O)C=C(Oc1ccc(C)cc1)c1ccc(OC)cc1</smiles>

1

$\mathbf{5 0 . 7 \% ~ Z - i s o m e r ~}$ 49.3\% E-isomer<smiles>CCCCOC(=O)C=C(Oc1ccc(OC)cc1)c1ccc(C(C)C)cc1</smiles>

2

$79.6 \%$ Z-isomer $20.4 \%$ E-isomer<smiles>CCCCOC(=O)C=C(Oc1ccc(Cl)cc1)c1ccc(C)cc1</smiles>

3

$81.0 \%$ Z-isomer $19.0 \%$ E-isomer<smiles>CCCOC(=O)C=C(Oc1ccc(C(C)C)cc1)c1ccc(C(C)=O)cc1</smiles>

$\mathbf{8 9 . 8} \%$ Z-isomer $10.2 \%$ E-isomer Catalyst (1 $\mathrm{mol} \%$ ) DMSO $(0.025 \mathrm{M})$ Blue LEDs, $20 \mathrm{~h}$<smiles>CCCCOC(=O)/C=C(/Oc1ccc(OC)cc1)c1ccc(C)cc1</smiles>

$(E)-1$<smiles>CCCOC(=O)/C=C(/Oc1ccc(OC)cc1)c1ccc(C(C)=O)cc1</smiles>

(E)-2<smiles>CCCCOC(=O)/C=C(/Oc1ccc(Cl)cc1)c1ccc(C)cc1</smiles>

(E)-3<smiles>CCCCOC(=O)/C=C(/Oc1ccc(C(C)=O)cc1)c1ccc(C(C)=O)cc1</smiles>

(E)-4

Table S3. Substrate generality screen with top four catalysts.

\begin{tabular}{cccc}
\hline Entry & Substrate & Catalyst & $\% \mathrm{E}$ \\
\hline 1 & $\mathbf{1}$ & $\operatorname{Ir}(\mathrm{ppy})_{3}$ & 95.8 \\
2 & $\mathbf{1}$ & $\operatorname{Ir}(\mathrm{dFppy})_{3}$ & 89.6 \\
3 & $\mathbf{1}$ & {$\left[\operatorname{Ir}\left(\mathrm{dF}\left(\mathrm{CF}_{3}\right) \mathrm{ppy}_{2}(\mathrm{dtbbpy}) \mathrm{PF}_{6}\right.\right.$} & 85.3 \\
4 & $\mathbf{1}$ & {$\left[\operatorname{Ir}(\mathrm{ppy})_{2}(\mathrm{dtbbpy}) \mathrm{PF}_{6}\right.$} & 94.2 \\
\hline 5 & $\mathbf{2}$ & $\operatorname{Ir}(\mathrm{ppy})_{3}$ & 88.2 \\
6 & $\mathbf{2}$ & $\operatorname{Ir}(\mathrm{dFppy})_{3}$ & 78.9 \\
7 & $\mathbf{2}$ & {$\left[\operatorname{Ir}\left(\mathrm{dF}\left(\mathrm{CF}_{3}\right) \mathrm{ppy}_{2}(\mathrm{dtbbpy})\right] \mathrm{PF}_{6}\right.$} & 79.2 \\
8 & $\mathbf{2}$ & {$\left[\operatorname{Ir}(\mathrm{ppy})_{2}(\mathrm{dtbbpy})\right] \mathrm{PF}_{6}$} & 93.4 \\
\hline 9 & $\mathbf{3}$ & $\operatorname{Ir}(\mathrm{ppy})_{3}$ & 91.1 \\
10 & $\mathbf{3}$ & $\operatorname{Ir}(\mathrm{dFppy})_{3}$ & 86.2
\end{tabular}




\begin{tabular}{cccc}
\hline Entry & Substrate & Catalyst & $\% \mathrm{E}$ \\
\hline 11 & $\mathbf{3}$ & {$\left[\operatorname{Ir}\left(\mathrm{dF}\left(\mathrm{CF}_{3}\right) \text { ppy }\right)_{2}(\mathrm{dtbbpy})\right] \mathrm{PF}_{6}$} & 83.4 \\
12 & $\mathbf{3}$ & {$\left[\operatorname{Ir}(\mathrm{ppy})_{2}(\mathrm{dtbbpy})\right] \mathrm{PF}_{6}$} & 93.4 \\
\hline 13 & $\mathbf{4}$ & $\operatorname{Ir}(\mathrm{ppy})_{3}$ & 82.9 \\
14 & $\mathbf{4}$ & $\operatorname{Ir}(\mathrm{dFppy})_{3}$ & 71.6 \\
15 & $\mathbf{4}$ & {$\left[\operatorname{Ir}\left(\mathrm{dF}\left(\mathrm{CF}_{3}\right) \mathrm{ppy}_{2}(\mathrm{dtbbpy}) \mathrm{PF}_{6}\right.\right.$} & 71.6 \\
16 & $\mathbf{4}$ & {$\left[\operatorname{Ir}(\mathrm{ppy})_{2}(\mathrm{dtbbpy})\right] \mathrm{PF}_{6}$} & 91.8 \\
\hline
\end{tabular}

The average \%E isomer can be correlated to triplet state energy of the catalyst:

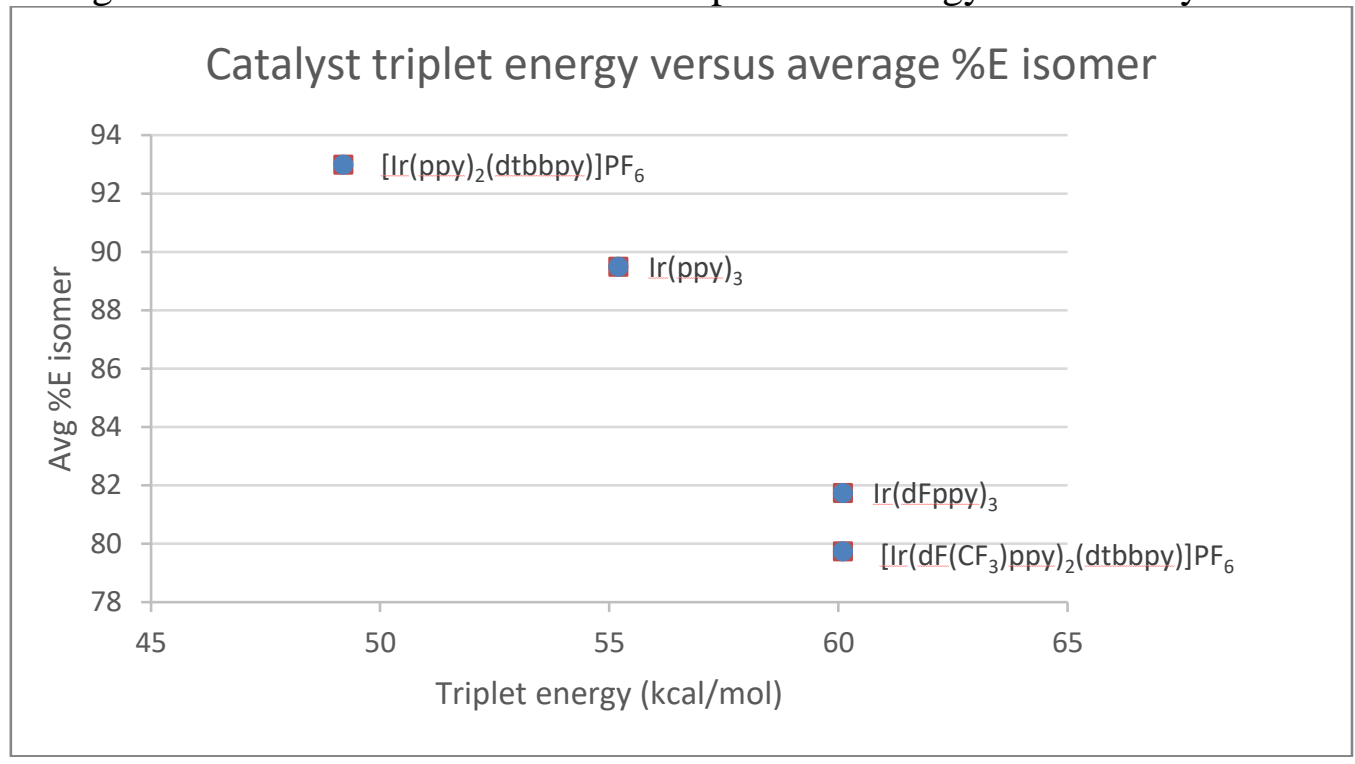

Figure S2. Comparison of average \% E isomer found in screen (Table S3) with the reported triplet state energy of each catalyst. ${ }^{2}$

\subsection{Light Source Comparison Study}

A catalyst stock solution as prepared from $\left[\operatorname{Ir}(\mathrm{ppy})_{2}(\mathrm{dtbbpy})\right] \mathrm{PF}_{6}(4.6 \mathrm{mg}, 5.0 \mu \mathrm{mol})$ and $1.0 \mathrm{ml}$ DMSO. To a vial containing $1(\mathrm{E} / \mathrm{Z}=47: 53,137 \mathrm{mg}, 0.40 \mathrm{mmol})$ and $3.4 \mathrm{~mL} \mathrm{DMSO}$ was added $40 \mu \mathrm{L}$ of the catalyst stock solution ( $0.05 \mathrm{~mol} \%$ loading). The prepared reaction solution was then divided (1.13 mL/vial) between three two-dram vials equipped with stir bars. The vials were irradiated with different light sources as described below and the reaction conversion was monitored by HPLC.

Description of each reaction assembly: The laser diode reaction set up was modified from a procedure by Harper et al. ${ }^{1}$ A 6W $450 \mathrm{~nm}$ laser diode (Amazon, ASIN = B014NN3AYW) equipped with a heat sink (Amazon, ASIN = B017BWKB2Q), was connected to a DC power source by alligator clips. The output power was controlled at the DC power supply by setting the amps to correspond to 2.0 watts of output as measured by a power meter (Thor Labs, part \# PM160T-HP). The lens on the laser was adjusted to produce a rectangle pattern $2.5 \mathrm{~cm} \times 0.1 \mathrm{~cm}$ at a distance of $8 \mathrm{~cm}$ from the laser. The reaction was placed below the laser at a distance of $8 \mathrm{~cm}$ from the reaction surface to the lens. Reactions were stirred at $600 \mathrm{rpm}$ open to air with temperature control provided by an aluminum block at RT (Figure S3a). The Kessil light set up is shown in Figure S3b; The vial was capped and 
placed $3.5 \mathrm{~cm}$ from the lamp (Kessil, part \# A160W). Temperature control was provided by a stream of air. The LED light set up is shown in Figure S3c; The vial was capped and placed in a crystallization dish with a diameter of approx. $10 \mathrm{~cm}$ surrounded by blue LEDs (www.superbrightleds.com, part \# NFLS-B300X3-WHT-BLUE). Temperature control was provided by a stream of air.

a)

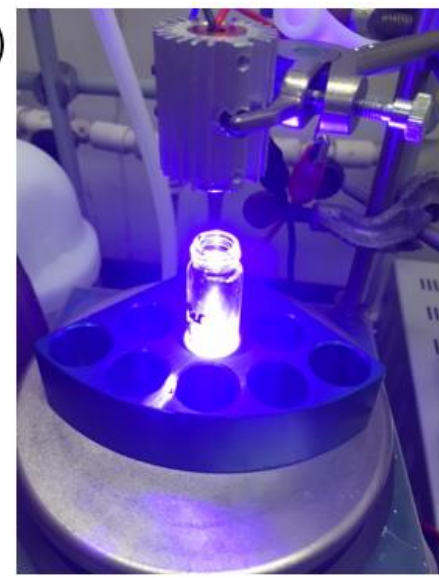

b)

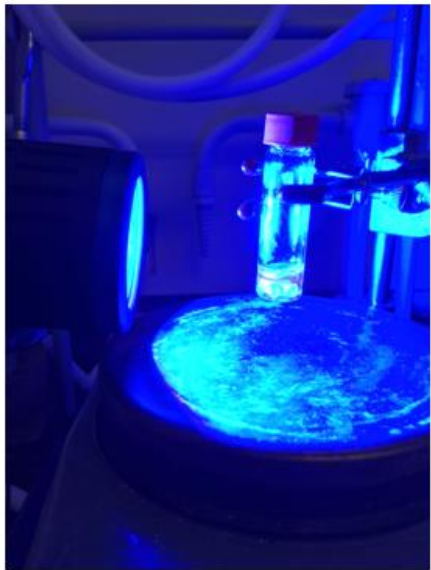

c)

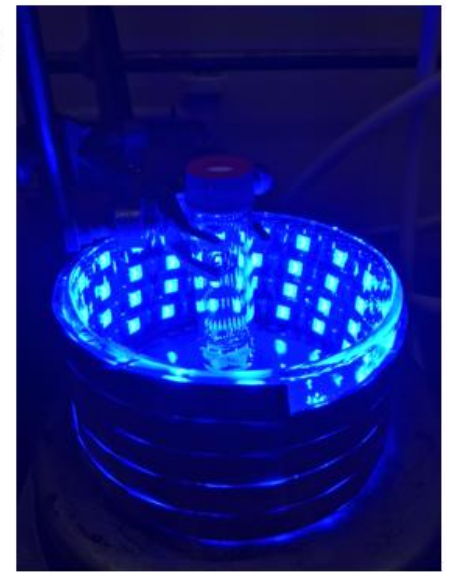

Figure S3. Reaction set up for light comparison study. A = Laser; B = Kessil Lamp; C = Blue LED strip

Table S4. Time course data for isomerization using three different light sources.

\begin{tabular}{cccc}
\hline time (min) & Laser, \% & Kessil lamp, \% & LED strip, \% \\
\hline 0 & 47.1 & 47.1 & 47.1 \\
5 & 54.7 & 48.1 & 47.2 \\
15 & 65.6 & 50.0 & 47.4 \\
25 & 72.9 & 51.8 & 47.6 \\
30 & 75.6 & 52.7 & 47.8 \\
45 & 82.3 & 55.1 & 48.1 \\
60 & 87.0 & 57.4 & 48.4 \\
130 & 92.6 & 66.4 & 49.9 \\
192 & 94.8 & 72.5 & 51.2 \\
300 & 94.9 & 79.9 & 53.4 \\
\hline
\end{tabular}

\subsection{Asymmetric Hydrogenation Ligand Screen}

In the glovebox, stock solutions of each ligand were prepared in $\mathrm{CH}_{2} \mathrm{Cl}_{2}(10 \mathrm{mM})$. The solutions were dispensed into $2 \mathrm{ml}$ vials containing a stir bar to give $0.22 \mu \mathrm{mol}$ of ligand per vial and the solvent was removed with a stream of air. A stock solution of bis(norbornadiene)rhodium(I) tetrafluoroborate was prepared in $\mathrm{CH}_{2} \mathrm{Cl}_{2}(0.75 \mathrm{mg} / \mathrm{mL}$ ) and dispensed to target $0.2 \mu \mathrm{mol}$ per vial $(100 \mu \mathrm{L})$. A stock solution of $(E)-3$ was prepared in $\mathrm{CH}_{2} \mathrm{Cl}_{2}(35 \mathrm{mg} / \mathrm{mL})$ and dispensed to target $10 \mu \mathrm{mol}$ per vial $(100 \mu \mathrm{L})$. The vials were capped, then pierced, before the rack of 48 vials was placed inside of a HEL Cat 96 reactor block and was pressurized to $8 \mathrm{~atm}$ of hydrogen and heated to $50{ }^{\circ} \mathrm{C}$ for $12 \mathrm{~h}$. 

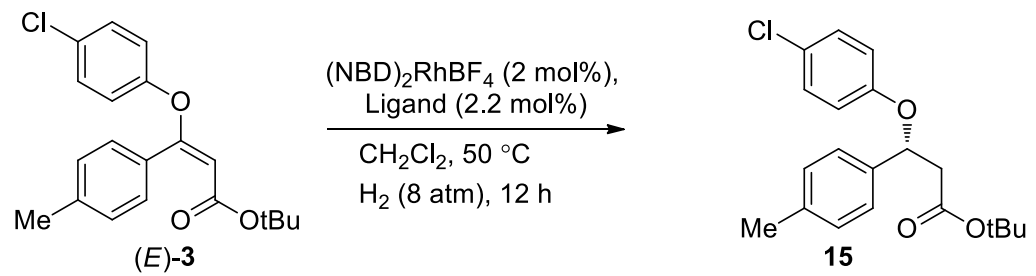

Table S5. Results of asymmetric hydrogenation HTE screen.

\begin{tabular}{|c|c|c|c|}
\hline Entry & ligand & Conv. & $\%$ ee \\
\hline 1 & J001-1 & 100 & 37.0 \\
\hline 2 & J002-1 & 100 & 30.8 \\
\hline 3 & J003-2 & 99.7 & -23.4 \\
\hline 4 & J004-2 & 95.9 & -24.3 \\
\hline 5 & J005-2 & 10.7 & -4.3 \\
\hline 6 & J006-1 & 100 & 58.4 \\
\hline 7 & J007-1 & 78.6 & 30.5 \\
\hline 8 & J008-2 & 14.5 & -7.6 \\
\hline 9 & J009-2 & 100 & -49.2 \\
\hline 10 & J011-1 & 100 & 32.8 \\
\hline 11 & J013-1 & 100 & 29.2 \\
\hline 12 & J015-1 & 17.7 & 22.0 \\
\hline 13 & J212-1 & 100 & 48.2 \\
\hline 14 & J216-2 & 100 & -65.2 \\
\hline 15 & J404-2 & 52.8 & -30.3 \\
\hline 16 & $\mathrm{~J} 418-2$ & 32.4 & -42.0 \\
\hline 17 & $\mathrm{~J} 425-2$ & 72 & -58.3 \\
\hline 18 & J452-1 & 21.7 & 39.2 \\
\hline 19 & $\mathrm{~J} 502-2$ & 100 & -83.8 \\
\hline 20 & J505-1 & 100 & 95.6 \\
\hline 21 & W001-1 & 100 & 86.0 \\
\hline 22 & W002-1 & 100 & 54.8 \\
\hline 23 & W003-1 & 100 & 21.7 \\
\hline 24 & W005-1 & 109 & 69.5 \\
\hline 25 & W006-1 & 100 & 33.8 \\
\hline 26 & W008-1 & 100 & 59.6 \\
\hline 27 & W009-1 & 100 & 25.1 \\
\hline 28 & W022-1 & 100 & -18.0 \\
\hline 29 & M001-1 & 34.6 & 8.1 \\
\hline 30 & M002-1 & 0 & NA \\
\hline 31 & M003-1 & 0 & NA \\
\hline 32 & M004-1 & 64.8 & 56.5 \\
\hline 33 & M009-1 & 47.5 & 49.5 \\
\hline 34 & M012-1 & 0 & NA \\
\hline 35 & T001-1 & 45.5 & 29.2 \\
\hline 36 & T002-1 & 0 & NA \\
\hline 37 & $\mathrm{~J} 210$ & 100 & 40.2 \\
\hline 38 & F356 & 0 & NA \\
\hline 39 & F131-1 & 0 & NA \\
\hline 40 & J403-1 & 21.8 & -20.2 \\
\hline 41 & $(S)$-PiP-Phos & 0 & NA \\
\hline 42 & $(R)$-BINAP & 22.6 & 47.8 \\
\hline 43 & $(R)$-DTBM-Segphos & 0 & NA \\
\hline 44 & $(S)$-Synphos & 24 & -30.8 \\
\hline
\end{tabular}




\begin{tabular}{cccc}
\hline Entry & ligand & Conv. & \% ee \\
\hline 45 & TCFP-Rh(BF $\left(\mathrm{BF}_{4}\right)$ & 44.9 & -2.4 \\
46 & $(R, R)$-QuinoxP & 47.5 & 32.2 \\
47 & Et-Phosphite & 0 & NA \\
48 & $(4-\mathrm{MeO})_{3} \mathrm{P}$ and $(R, R)$-IPr-Duphos & 2.8 & -14.3 \\
\hline
\end{tabular}




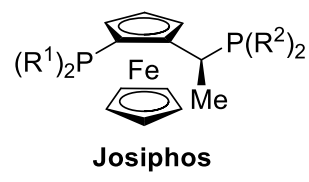

J001-1: $R^{1}=P h, R^{2}=C y$

J002-1: $\mathrm{R}^{1}=\mathrm{Ph}, \mathrm{R}^{2}=t-\mathrm{Bu}$

J006-1: $R^{1}=3,5-\mathrm{CF}_{3}-\mathrm{Ph}, \mathrm{R}^{2}=\mathrm{Cy}$

J007-1: $R^{1}=3,5-\mathrm{CF}_{3}-4-\mathrm{OMe}-\mathrm{Ph}, \mathrm{R}^{2}=\mathrm{Cy}$

J011-1: $R^{1}=t-B u, R^{2}=4-C_{3}-P h$

J013-1: $\mathrm{R}^{1}=4-\mathrm{CF}_{3}-\mathrm{Ph}, \mathrm{R}^{2}=t-\mathrm{Bu}$

J015-1: $R^{1}=$ Furyl, $R^{2}=3,5-M e-P h$

J212-1: $R^{1}=$ Furyl, $R^{2}=t-B u$

J403-1: $R^{1}=4-\mathrm{CF}_{3}-\mathrm{Ph}, \mathrm{R}^{2}=4-\mathrm{CF}_{3}-\mathrm{Ph}$

J452-1: $R^{1}=$ Furyl, $R^{2}=2-M e-P h$

$J 505-1: R^{1}=t-B u, R^{2}=2-M e-P h$

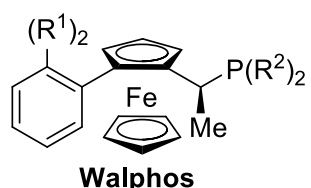

W001-1: $\mathrm{R}^{1}=\mathrm{Ph}, \mathrm{R}^{2}=3,5-\mathrm{CF}_{3}-\mathrm{Ph}$

W002-1: $R^{1}=P h, R^{2}=P h$

W003-1: $R^{1}=P h, R^{2}=C y$

W005-1: $\mathrm{R}^{1}=3,4-\mathrm{CH}_{3}-4-\mathrm{OMe}-\mathrm{Ph}, \mathrm{R}^{2}=3,5-\mathrm{CF}_{3}-\mathrm{Ph}$

W006-1: $\mathrm{R}^{1}=\mathrm{Ph}, \mathrm{R}^{2}=3,5-\mathrm{CH}_{3}-\mathrm{Ph}$

W008-1: $R^{1}=\mathrm{Cy}, \mathrm{R}^{2}=3,5-\mathrm{CF}_{3}-\mathrm{Ph}$

W009-1: $\mathrm{R}^{1}=3,5-\mathrm{CH}_{3}-\mathrm{Ph}, \mathrm{R}^{2}=3,5-\mathrm{CH}_{3}-\mathrm{Ph}$

W022-1: $R^{1}=P h, R^{2}=$ Norbornyl

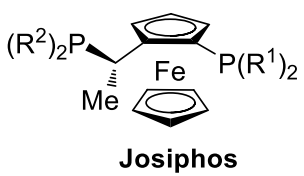

J003-2: $R^{1}=C y, R^{2}=C y$

J004-2: $R^{1}=C y, R^{2}=P h$

J005-2: $R^{1}=P h, R^{2}=3,5-M e-P h$

J008-2: $R^{1}=3,5-C_{3}-P h, R^{2}=3,5-M e-P h$

J009-2: $R^{1}=C y, R^{2}=t-B u$

J216-2: $\mathrm{R}^{1}=$ Napthyl, $\mathrm{R}^{2}=t-\mathrm{Bu}$

J404-2: $R^{1}=$ Napthyl, $R^{2}=3,5-M e-P h$

J418-2: $R^{1}=3,5-M e-4-O M e-P h, R^{2}=3,5-M \epsilon$

J425-2: $R^{1}=3,5-M e-4-O M e-P h, R^{2}=2-M e-F$

J502-2: $\mathrm{R}^{1}=t-\mathrm{Bu}, \mathrm{R}^{2}=\mathrm{Ph}$

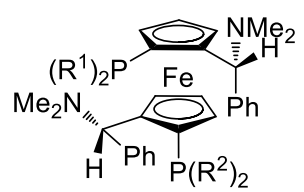

Mandyphos

M001-1: $R^{1}=R^{2}=P h$

M002-1: $R^{1}=R^{2}=C y$

M003-1: $\mathrm{R}^{1}=\mathrm{R}^{2}=3,5-\mathrm{CF}_{3}-\mathrm{Ph}$

M004-1: $\mathrm{R}^{1}=\mathrm{R}^{2}=3,5-\mathrm{CH}_{3}-4-\mathrm{OMe}-\mathrm{Pr}$

M009-1: $R^{1}=R^{2}=3,5-\mathrm{CH}_{3}-\mathrm{Ph}$

M012-1: $R^{1}=R^{2}=2-\mathrm{CH}_{3}-\mathrm{Ph}$
$\left(R^{1}\right)_{2}$

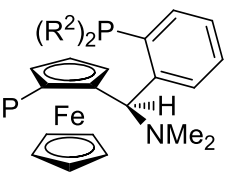

Taniaphos

T001-1: $R^{1}=R^{2}=P h$

T002-1: $R^{1}=R^{2}=C y$

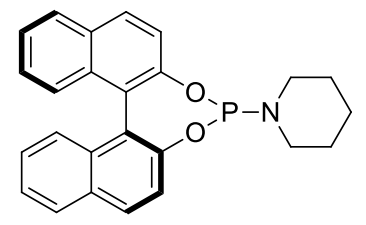

(S)-PiP-Phos<smiles>c1ccc(-c2ccccc2-c2ccc3c(c2-c2ccccc2)OCCO3)cc1</smiles>

(S)-Synphos
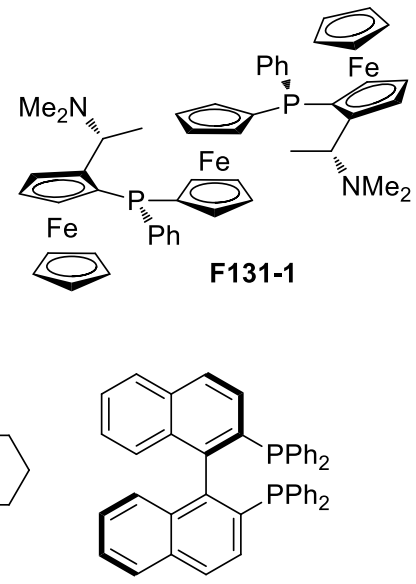

$(R)$-Binap

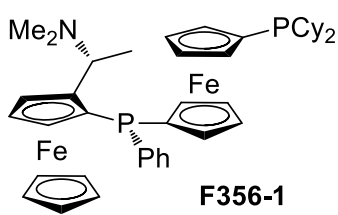<smiles>[R20]c1ccc2c(c1-c1c(P)ccc3c1OCO3)OCO2</smiles><smiles>[R7]c1cc(C(C)(C)C)c(OC)c(C(C)(C)C)c1</smiles>

$(R)$-DTBM-Segphos

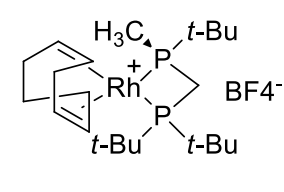

TCFP-Rh(BF4)

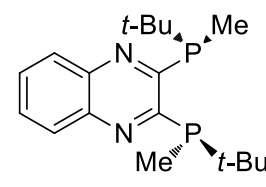

$(R, R)$-QuinoxP<smiles>CC(C)C1CCC(C(C)C)P1c1ccccc1P1C(C(C)C)CCC1C(C)(C)C</smiles>

$(R, R)$-iPr-DuPhos 
Figure S4. Structures of ligands used in asymmetric hydrogenation screen.

\subsection{Solvent Screen for Asymmetric Hydrogenation}
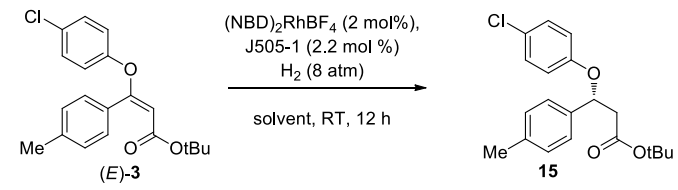

Table S6. Results of asymmetric hydrogenation in different solvents.

\begin{tabular}{ccc}
\hline Solvent & Conversion & \% ee \\
\hline DME & 18.1 & 96.1 \\
THF & 99.0 & 95.9 \\
Dioxane & 56.8 & 93.3 \\
Toluene & 79.2 & 95.2 \\
DCM & 99.7 & 96.8 \\
Propylene Carbonate & 86.0 & 94.7 \\
MeOH & 77.0 & 95.7 \\
DCM:MeOH (3:1) & 98.6 & 96.5 \\
EtOH & 80.8 & 95.8 \\
IPA & 91.8 & 95.6 \\
2-MeTHF & 71.1 & 96.2 \\
EtOAc & 26.9 & 95.3 \\
2,2,2-Trifluoroethanol & 99.7 & 95.7 \\
Heptane & 9.4 & 91.1 \\
\hline
\end{tabular}

Note: although several solvents gave acceptable results, $\mathrm{CH}_{2} \mathrm{Cl}_{2}$ was chosen for ease of aqueous work up and isolation.

\section{General Procedures}

\subsection{Starting Material Synthesis}

Synthesis of alkynyl esters. Alkynyl esters were synthesized either by Method A or Method B, as described below.

\section{Method A:}

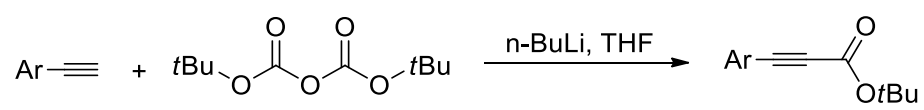

In a modified procedure by Kotora, ${ }^{3}$ alkyne ( 1 equiv) was charged to round-bottom flask equipped with a magnetic bar then capped with a rubber septum. The flask was purged with nitrogen for approximately $15 \mathrm{~min}$. Tetrahydrofuran $(\sim 15 \mathrm{~mL} / \mathrm{g}$ alkyne $)$ was added and the solution was cooled to $-78{ }^{\circ} \mathrm{C}$ by using a dry-ice/acetone bath. $n$-BuLi (1.6 M in hexanes, 1.1 equiv) was added dropwise and mixed for 2 hours under a slight positive pressure of argon. Di-tert-butyl dicarbonate (1.2 equiv) was dissolved in tetrahydrofuran ( $4 \mathrm{~mL} / \mathrm{g}$ di-tert-butyl dicarbonate) and was added dropwise to the above solution with a syringe. The solution was allowed to warm up to room temperature. The reaction mixture was transferred to a separatory funnel and washed with $10 \mathrm{wt} \%$ aqueous $\mathrm{NaCl}$ solution $(10 \mathrm{~mL} / \mathrm{g}$ alkyne). The aqueous layer was back extracted with dichloromethane $(10 \mathrm{~mL} / \mathrm{g}$ alkyne). The combined layer was filtered through celite and was concentrated in vacuo. The crude product was purified by silica gel column chromatography. 


\section{Method B:}

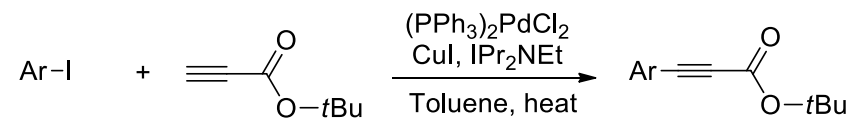

Following literature reports, ${ }^{4,5}$ a round-bottom flask was charged with aryl iodide (1 equiv), bis(triphenylphosphine)palladium(II) dichloride (0.75 mol \%), copper(I) iodide (1.5 mol $\%$ ) and potassium carbonate ( 3 equiv). The flask was purged with argon for approximately 15 min. In a separate flask, alkyne (1.5 equiv) was dissolved in 2-MeTHF (0.6 M) and purged with argon for 10 mins before transferring solution to flask containing catalyst, base and aryl iodide. The reaction mixture was heated to $75^{\circ} \mathrm{C}$ using a heating mantle and let stir overnight. The reaction mixture was transferred to a separatory funnel and washed with $1 \mathrm{M}$ aqueous $\mathrm{LiCl}$ solution $(12 \mathrm{~mL} / \mathrm{g}$ aryl iodide $\times 2)$. The organic layer was dried over sodium sulfate and concentrated in vacuo to give the crude product that was purified by silica gel column chromatography.

\subsection{Oxa-Michael, Isomerization, and Reduction Standard Procedures}

Synthesis of enantioenriched $\boldsymbol{\beta}$-aryloxyesters. The esters were synthesized by the sequence of Method C, D and E described below. The product from Method C was used without purification. The products from Method D and E were purified and characterized as follows.

\section{Method C:}

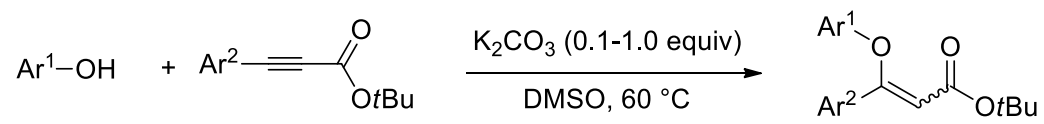

To a vial was added alkyne (1.0 equiv), phenol (1.05 equiv) and potassium carbonate ( 0.1 equiv or 1.0 equiv), diluted with DMSO $(1.0 \mathrm{M})$ and stirred at $65^{\circ} \mathrm{C}$ on a heating mantle until alkyne was no longer detected by HPLC. The reaction mixture was diluted with MTBE $(10 \mathrm{~mL})$ and $0.5 \mathrm{M}$ aq $\mathrm{LiCl}(10 \mathrm{~mL})$. The resulting organic layer was washed again with $0.5 \mathrm{M}$ aq $\mathrm{LiCl}$ and then dried over $\mathrm{MgSO}_{4}$ and concentrated in vacuo.

\section{Method D:}
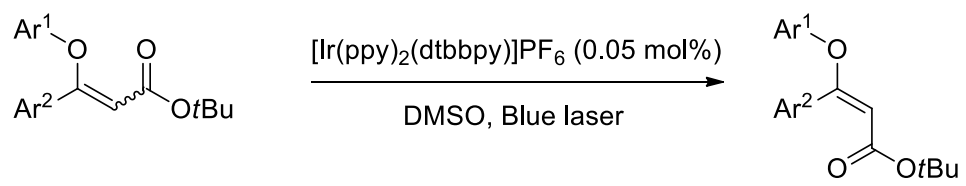

To the crude oil obtained in Method $\mathrm{C}$ was added $\left[\operatorname{Ir}(\mathrm{ppy})_{2}(\mathrm{dtbbpy})\right] \mathrm{PF}_{6}(0.0005$ equiv, added as a $4.5 \mathrm{mg} / \mathrm{mL}$ stock solution in DMSO) and the reaction was diluted with DMSO $(0.2 \mathrm{M})$. The reaction mixture was set to stir under $2 \mathrm{~W}$ blue $(459 \mathrm{~nm}$, see emission spectrum below, Figure S5) laser irradiation as described by Harper et $\mathrm{al}^{1}$ and in the above light comparison experiment (Section 2.4, Figure S3a), until photostationary state was reached (no change in $\mathrm{E} / \mathrm{Z}$ ratio over time). The reaction mixture was diluted with MTBE $(10 \mathrm{~mL})$ and $0.5 \mathrm{M}$ aq $\mathrm{LiCl}(10 \mathrm{~mL})$. The resulting organic layer was washed with $0.5 \mathrm{M}$ aq $\mathrm{LiCl}$ $(10 \mathrm{~mL})$, dried over $\mathrm{MgSO}_{4}$, and concentrated in vacuo. The product was purified via 
column chromatography and crystallization from heptane or pentane to give pure olefin isomers.

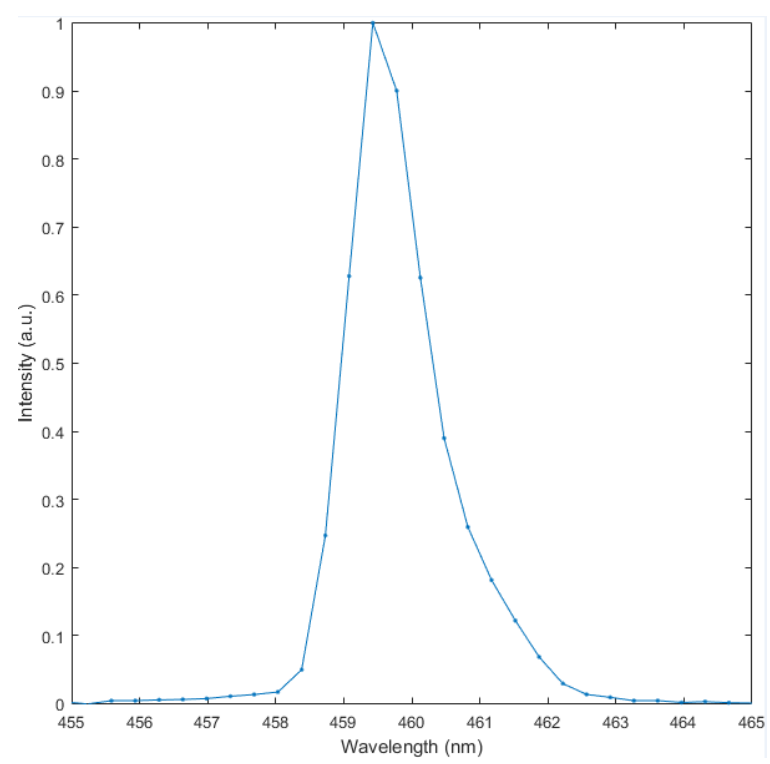

Figure S5. Emission spectrum of laser diode at room temperature, attenuated with an OD 3 Neutral Density filter and collected with a 0.22 NA 200 micron diameter UVVIS fiber optic into an Ocean Insight Flame UV-VIS spectrometer.

Note: Method C and D can be run in one pot without the intermediate work up if the oxaMichael is run under air free conditions to prevent the formation of dark colored intermediates. The isomerization reaction also requires a higher catalyst loading. For this reason, an intermediate work up was performed.

\section{Method E:}

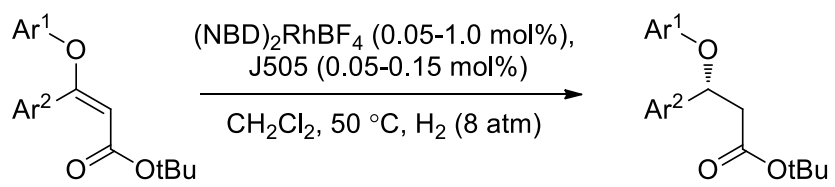

Catalyst stock solution preparation: The catalyst stock solution was prepared by dissolving bis(norbornadiene)rhodium(I) tetrafluoroborate $(0.24 \mu \mathrm{mol} / \mathrm{mL})$ and $\mathrm{J505-1}(0.26$ $\mu \mathrm{mol} / \mathrm{mL}$ ) in $\mathrm{CH}_{2} \mathrm{Cl}_{2}$ and stirring at $\mathrm{RT}$ for 15 minutes.

Reaction: To a vial containing the olefin ( 1.0 equiv) was added the catalyst $(0.05-1.0 \mathrm{~mol}$ $\%$ ) prepared as a stock solution in $\mathrm{CH}_{2} \mathrm{Cl}_{2}$, as described above. The reaction was pressurized to $8 \mathrm{~atm}$ of hydrogen and let stir at RT for $16 \mathrm{~h}$. The reaction mixture was concentrated in vacuo and the crude oil was purified via column chromatography.

\section{Characterization Data for Products}




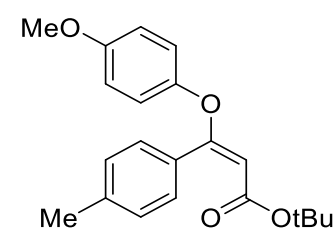

tert-Butyl (E)-3-(4-methoxyphenoxy)-3-(p-tolyl)acrylate $((E)-1)$. Following Method $\mathrm{C}$, a mixture of tert-butyl 3-(p-tolyl)propiolate $(0.216 \mathrm{~g}, 1 \mathrm{mmol}), 4$-methoxyphenol $(0.130 \mathrm{~g}$, $1.050 \mathrm{mmol})$, potassium carbonate $(0.014 \mathrm{~g}, 0.100 \mathrm{mmol})$ in DMSO $(1.0 \mathrm{~mL})$ was stirred at $60{ }^{\circ} \mathrm{C}$ for $1 \mathrm{~h}$. Crude HPLC analysis showed E/Z ratio $=37: 63$. Following Method D, the crude oil and $\left[\operatorname{Ir}(\mathrm{ppy})_{2}(\mathrm{dtbbpy})\right] \mathrm{PF}_{6}(0.45 \mathrm{mg}, 0.50 \mu \mathrm{mol})$ were stirred in DMSO (5.0 $\mathrm{mL}$ ) under laser irradiation for $6 \mathrm{~h}$. The crude product was purified via flash column chromatography (20\% EtOAc/heptanes) to provide the title compound as a white solid (0.296 g, 87\%, 96:4 E/Z ratio). Recrystallization from heptanes (6 mL) afforded pure $\mathrm{E}$ isomer as a white solid (252 $\mathrm{mg}, 74 \%$ yield). $\mathrm{mp} 136.7-138.9^{\circ} \mathrm{C}$;

${ }^{1} \mathrm{H}$ NMR (400 MHz, Chloroform- $d$ ) $\delta 7.48(\mathrm{~d}, J=7.9 \mathrm{~Hz}, 2 \mathrm{H}), 7.22(\mathrm{~d}, J=7.8 \mathrm{~Hz}, 2 \mathrm{H})$, $7.08-7.00(\mathrm{~m}, 2 \mathrm{H}), 6.91(\mathrm{~d}, J=9.0 \mathrm{~Hz}, 2 \mathrm{H}), 5.02(\mathrm{~s}, 1 \mathrm{H}), 3.82(\mathrm{~s}, 3 \mathrm{H}), 2.39(\mathrm{~s}, 3 \mathrm{H}), 1.31$ (s, 9H); ${ }^{13} \mathrm{C}$ NMR (101 MHz, Chloroform-d) $\delta 170.0$ (C), 166.4 (C), 157.1 (C), 147.7 (C), $140.1(\mathrm{C}), 131.6(\mathrm{C}), 129.3(\mathrm{CH}), 128.6(\mathrm{CH}), 122.5(\mathrm{CH}), 115.1(\mathrm{CH}), 99.4(\mathrm{CH}), 79.9$ (C), $55.8\left(\mathrm{CH}_{3}\right), 28.2\left(\mathrm{CH}_{3}\right), 21.7\left(\mathrm{CH}_{3}\right)$; HRMS (TOF MS ES+) $m / z:[\mathrm{M}+\mathrm{H}]^{+}$calcd for $\mathrm{C}_{21} \mathrm{H}_{25} \mathrm{O}_{4}$ 341.1747; found 341.1755.

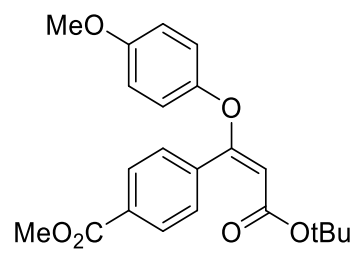

Methyl (E)-4-(3-(tert-butoxy)-1-(4-methoxyphenoxy)-3-oxoprop-1-en-1-yl)benzoate ((E)-2). Following Method C, a mixture of methyl 4-(3-(tert-butoxy)-3-oxoprop-1-yn-1yl)benzoate $(0.260 \mathrm{~g}, 1 \mathrm{mmol})$, 4-methoxyphenol $(0.130 \mathrm{~g}, 1.050 \mathrm{mmol})$, potassium carbonate $(0.014 \mathrm{~g}, 0.100 \mathrm{mmol})$ in DMSO $(1.0 \mathrm{~mL})$ was stirred at $60{ }^{\circ} \mathrm{C}$ for $1 \mathrm{~h}$. Crude HPLC analysis showed $\mathrm{E} / \mathrm{Z}$ ratio $=24: 76$. Following Method $\mathrm{D}$, the crude oil and $\left[\operatorname{Ir}(\text { ppy })_{2}(\mathrm{dtbbpy})\right] \mathrm{PF}_{6}(0.45 \mathrm{mg}, 0.50 \mu \mathrm{mol})$ were stirred in DMSO $(5.0 \mathrm{~mL})$ under laser irradiation for $6 \mathrm{~h}$. The crude product was purified via flash column chromatography $(20 \%$ EtOAc/heptanes) to provide the title compound as a white solid $(0.320 \mathrm{~g}, 83 \%, 97: 3 \mathrm{E} / \mathrm{Z}$ ratio). Recrystallization from Heptanes $(6 \mathrm{~mL})$ afforded pure E isomer as a white solid (274 mg, $71 \%$ yield). mp $111.2-112.5{ }^{\circ} \mathrm{C} ;{ }^{1} \mathrm{H}$ NMR $(700 \mathrm{MHz}$, Chloroform- $d$ ) $\delta 8.11-8.07$ (m, $2 \mathrm{H}), 7.68-7.62(\mathrm{~m}, 2 \mathrm{H}), 7.09-7.02(\mathrm{~m}, 2 \mathrm{H}), 6.96-6.89(\mathrm{~m}, 2 \mathrm{H}), 5.11(\mathrm{~s}, 1 \mathrm{H}), 3.94(\mathrm{~s}$, $3 \mathrm{H}), 3.82(\mathrm{~s}, 3 \mathrm{H}), 1.28(\mathrm{~s}, 9 \mathrm{H}) ;{ }^{13} \mathrm{C}$ NMR (176 MHz, Chloroform- $d$ ) $\delta 168.6(\mathrm{C}), 166.8$ (C), $165.9(\mathrm{C}), 157.3(\mathrm{C}), 147.3(\mathrm{C}), 139.2(\mathrm{C}), 131.2(\mathrm{C}), 129.4(\mathrm{CH}), 129.2(\mathrm{CH}), 122.4$ $(\mathrm{CH}), 115.2(\mathrm{CH}), 100.8(\mathrm{CH}), 80.3(\mathrm{C}), 55.8\left(\mathrm{CH}_{3}\right), 52.4\left(\mathrm{CH}_{3}\right), 28.1\left(\mathrm{CH}_{3}\right)$; Elemental analysis calcd for $\mathrm{C}_{22} \mathrm{H}_{24} \mathrm{O}_{6}$ : $\mathrm{C}(68.74 \%), \mathrm{H}(6.29 \%)$; found $\mathrm{C}(68.80 \%), \mathrm{H}(6.45 \%)$.

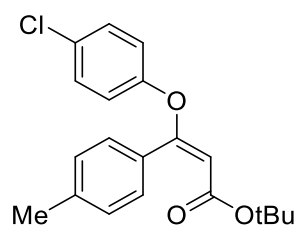


tert-Butyl (E)-3-(4-chlorophenoxy)-3-(p-tolyl)acrylate ((E)-3). Following Method C, a mixture of tert-butyl 3-(p-tolyl)propiolate $(0.199 \mathrm{~g}, 0.92 \mathrm{mmol})$, 4-chlorophenol $(0.130 \mathrm{~g}$, $1.012 \mathrm{mmol})$, and potassium carbonate $(0.127 \mathrm{~g}, 0.920 \mathrm{mmol})$ in DMSO $(1.0 \mathrm{~mL})$ was stirred at $60{ }^{\circ} \mathrm{C}$ for $16 \mathrm{~h}$. Crude HPLC analysis showed E/Z ratio $=20: 80$. Following Method D, the crude oil and $\left[\operatorname{Ir}(\mathrm{ppy})_{2}(\mathrm{dtbbpy})\right] \mathrm{PF}_{6}(0.42 \mathrm{mg}, 0.46 \mu \mathrm{mol})$ were stirred in DMSO $(4.6 \mathrm{~mL})$ under laser irradiation for $3 \mathrm{~h}$. The crude product was purified via flash column chromatography (20\% EtOAc/heptanes) to provide the title compound as a white solid $(0.274 \mathrm{~g}, 86 \%, 95: 5 \mathrm{E} / \mathrm{Z}$ ratio). Recrystallized from heptanes $(3 \mathrm{~mL})$ to afford pure $\mathrm{E}$ isomer as a white solid $(231 \mathrm{mg}, 73 \%$ yield $) . \mathrm{mp}$ 94.9-95.6 ${ }^{\circ} \mathrm{C} ;{ }^{1} \mathrm{H}$ NMR $(700 \mathrm{MHz}$, Chloroform- $d$ ) $\delta 7.46(\mathrm{~d}, J=7.8 \mathrm{~Hz}, 2 \mathrm{H}), 7.36-7.32(\mathrm{~m}, 2 \mathrm{H}), 7.21(\mathrm{~d}, J=7.8 \mathrm{~Hz}, 2 \mathrm{H})$, $7.06-7.03(\mathrm{~m}, 2 \mathrm{H}), 5.12(\mathrm{~s}, 1 \mathrm{H}), 2.38(\mathrm{~s}, 3 \mathrm{H}), 1.33(\mathrm{~s}, 9 \mathrm{H}) ;{ }^{13} \mathrm{C}$ NMR $(176 \mathrm{MHz}$, Chloroform- $d$ ) 168.5 (C), 166.0 (C), 153.1 (C), 140.4 (C), 130.9 (C), 130.5 (C), 130.1 $(\mathrm{CH}), 129.3(\mathrm{CH}), 128.7(\mathrm{CH}), 122.6(\mathrm{CH}), 101.3(\mathrm{CH}), 80.2(\mathrm{C}), 28.1\left(\mathrm{CH}_{3}\right), 21.7\left(\mathrm{CH}_{3}\right)$; HRMS (TOF) $m / z:[\mathrm{M}+\mathrm{H}]^{+}$calcd for $\mathrm{C}_{20} \mathrm{H}_{22} \mathrm{ClO}_{3} 345.1251$; found 345.1266.

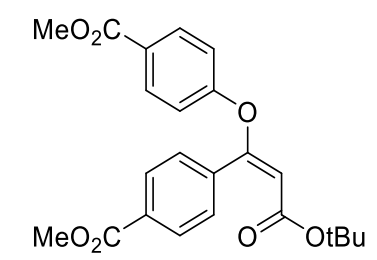

Methyl (E)-4-(3-(tert-butoxy)-1-(4-(methoxycarbonyl)phenoxy)-3-oxoprop-1-en-1yl)benzoate $((E)-4)$. Following Method C, a mixture of methyl 4-(3-(tert-butoxy)-3oxoprop-1-yn-1-yl)benzoate $(0.260 \mathrm{~g}, 1.00 \mathrm{mmol})$, methyl 4-hydroxybenzoate $(0.160 \mathrm{~g}$, $1.05 \mathrm{mmol})$ and potassium carbonate $(0.014 \mathrm{~g}, 0.10 \mathrm{mmol})$ in DMSO $(1.00 \mathrm{~mL})$ was stirred at $60{ }^{\circ} \mathrm{C}$ for $4 \mathrm{~h}$. Crude HPLC analysis showed $\mathrm{E} / \mathrm{Z}$ ratio $=15: 85$. Following Method D, the crude oil and $\left[\operatorname{Ir}(\text { ppy })_{2}(\mathrm{dtbbpy})\right] \mathrm{PF}_{6}(0.45 \mathrm{mg}, 0.50 \mu \mathrm{mol})$ were stirred in DMSO (5.0 $\mathrm{mL}$ ) under laser irradiation for $5 \mathrm{~h}$. The crude product was purified via flash column chromatography (20\% EtOAc/heptanes) to provide the title compound as a white solid (369 $\mathrm{mg}, 89 \%, 94: 6 \mathrm{E} / \mathrm{Z}$ ratio). Recrystallized from heptanes $(6 \mathrm{~mL})$ to afford pure $\mathrm{E}$ isomer as a white solid (322 mg, 78\% yield). mp 123.7-127.2 ${ }^{\circ} \mathrm{C}$; ${ }^{1} \mathrm{H}$ NMR (700 MHz, Chloroformd) $\delta 8.10-8.03(\mathrm{~m}, 4 \mathrm{H}), 7.66-7.62(\mathrm{~m}, 2 \mathrm{H}), 7.19-7.13(\mathrm{~m}, 2 \mathrm{H}), 5.33(\mathrm{~s}, 1 \mathrm{H}), 3.93(\mathrm{~s}$, $3 \mathrm{H}), 3.91(\mathrm{~s}, 3 \mathrm{H}), 1.31(\mathrm{~s}, 9 \mathrm{H}) ;{ }^{13} \mathrm{C}$ NMR $(176 \mathrm{MHz}$, Chloroform-d) $\delta 166.6(\mathrm{C}), 166.4$ (C), $166.0(\mathrm{C}), 165.2(\mathrm{C}), 158.2(\mathrm{C}), 138.1(\mathrm{C}), 132.0(\mathrm{CH}), 131.5(\mathrm{C}), 129.5(\mathrm{CH}), 129.3$ $(\mathrm{CH}), 127.2(\mathrm{C}), 120.7(\mathrm{CH}), 104.3(\mathrm{CH}), 80.9(\mathrm{C}), 52.4\left(\mathrm{CH}_{3}\right), 52.4\left(\mathrm{CH}_{3}\right), 28.1\left(\mathrm{CH}_{3}\right)$; Elemental analysis calcd for $\mathrm{C}_{23} \mathrm{H}_{24} \mathrm{O}_{7}$ : $\mathrm{C}(66.98 \%), \mathrm{H}(5.87 \%)$; found $\mathrm{C}(66.94 \%), \mathrm{H}$ $(5.96 \%)$.

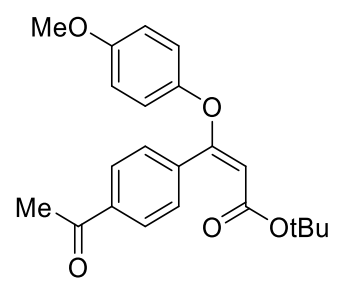

tert-Butyl (E)-3-(4-acetylphenyl)-3-(4-methoxyphenoxy)acrylate $\quad((E)-5)$. Following Method C, a mixture of tert-butyl 3-(4-acetylphenyl)propiolate (244 mg, $1.0 \mathrm{mmol})$, 4methoxyphenol $(0.130 \mathrm{~g}, 1.050 \mathrm{mmol})$, potassium carbonate $(0.014 \mathrm{~g}, 0.100 \mathrm{mmol})$ in 
DMSO $(1.0 \mathrm{~mL})$ was stirred at $60{ }^{\circ} \mathrm{C}$ for $1 \mathrm{~h}$. Crude HPLC analysis showed E/Z ratio $=$ 31:69. Following Method D, the crude oil and $\left[\operatorname{Ir}(\mathrm{ppy})_{2}(\mathrm{dtbbpy})\right] \mathrm{PF}_{6}(0.45 \mathrm{mg}, 0.5 \mu \mathrm{mol})$ were stirred in a mixture of DMSO $(4.0 \mathrm{~mL})$ and acetone $(3.0 \mathrm{~mL})$ under laser irradiation for $3 \mathrm{~h}$. The crude product was purified via flash column chromatography (20\% EtOAc/heptanes) to provide the title compound as a white solid (0.305 g, 82\%, 96:4 E/Z ratio). Recrystallization from Heptanes $(6 \mathrm{~mL})$ afforded pure E isomer as a white solid (270 mg, 73\% yield). mp 125.0-126.7 ${ }^{\circ} \mathrm{C} ;{ }^{1} \mathrm{H}$ NMR (700 MHz, Chloroform- $d$ ) $\delta 8.07-7.95$ (m, $2 \mathrm{H}), 7.73-7.62(\mathrm{~m}, 2 \mathrm{H}), 7.11-7.00(\mathrm{~m}, 2 \mathrm{H}), 6.99-6.87(\mathrm{~m}, 2 \mathrm{H}), 5.12(\mathrm{~s}, 1 \mathrm{H}), 3.82(\mathrm{~s}$, 3H), 2.63 (s, 3H), 1.30 (s, 9H); ${ }^{13} \mathrm{C}$ NMR (176 MHz, Chloroform-d) $\delta 197.7$ (C), 168.6 (C), $165.9(\mathrm{C}), 157.3(\mathrm{C}), 147.3(\mathrm{C}), 139.2(\mathrm{C}), 137.9(\mathrm{C}), 129.6(\mathrm{CH}), 127.9(\mathrm{CH}), 122.4$ $(\mathrm{CH}), 115.2(\mathrm{CH}), 100.7(\mathrm{CH}), 80.3(\mathrm{C}), 55.8\left(\mathrm{CH}_{3}\right), 28.1\left(\mathrm{CH}_{3}\right), 26.9\left(\mathrm{CH}_{3}\right)$; Elemental analysis calcd for $\mathrm{C}_{22} \mathrm{H}_{24} \mathrm{O}_{5}$ : C (71.72\%), $\mathrm{H}(6.57 \%)$; found $\mathrm{C}(71.84 \%), \mathrm{H}(6.87 \%)$.

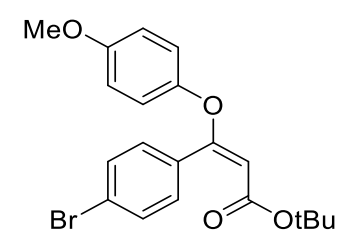

tert-Butyl (E)-3-(4-bromophenyl)-3-(4-methoxyphenoxy)acrylate ((E)-6). Following Method C, a mixture of methyl tert-butyl 3-(4-bromophenyl)propiolate (281 mg, 1.0 $\mathrm{mmol})$, 4-methoxyphenol $(0.130 \mathrm{~g}, 1.050 \mathrm{mmol})$, potassium carbonate $(0.014 \mathrm{~g}, 0.100$ mmol) in DMSO $(1.0 \mathrm{~mL})$ was stirred at $60{ }^{\circ} \mathrm{C}$ for $1 \mathrm{~h}$. Crude HPLC analysis showed E/Z ratio $=31: 69$. Following Method $\mathrm{D}$, the crude oil and $\left[\operatorname{Ir}(\mathrm{ppy})_{2}(\mathrm{dtbbpy})\right] \mathrm{PF}_{6}(0.45 \mathrm{mg}, 0.50$ $\mu \mathrm{mol})$ were stirred in a mixture of DMSO $(4.0 \mathrm{~mL})$ and acetone $(3.0 \mathrm{~mL})$ under laser irradiation for $3 \mathrm{~h}$. The crude product was purified via flash column chromatography ( $20 \%$ EtOAc/heptanes) to provide the title compound as a white solid $(0.353 \mathrm{~g}, 87 \%, 99: 1 \mathrm{E} / \mathrm{Z}$ ratio). Recrystallization from Heptanes $(6 \mathrm{~mL})$ afforded pure E isomer as a white solid (325 $\mathrm{mg}, 80 \%$ yield). mp $157.6-159.3^{\circ} \mathrm{C}$; ${ }^{1} \mathrm{H}$ NMR (400 MHz, Chloroform- $d$ ) $\delta 7.57-7.53$ (m, $2 \mathrm{H}), 7.49-7.44(\mathrm{~m}, 2 \mathrm{H}), 7.10-6.98(\mathrm{~m}, 2 \mathrm{H}), 6.98-6.82(\mathrm{~m}, 2 \mathrm{H}), 5.06(\mathrm{~s}, 1 \mathrm{H}), 3.82(\mathrm{~s}$, 3H), 1.31 (s, 9H); ${ }^{13} \mathrm{C}$ NMR (101 MHz, Chloroform- $d$ ) $\delta 170.0$ (C), 166.4 (C), 157.1 (C), 147.7 (C), $140.1(\mathrm{C}), 131.6(\mathrm{C}), 129.3(\mathrm{CH}), 128.6(\mathrm{CH}), 122.5(\mathrm{CH}), 115.1(\mathrm{CH}), 99.4$ (C), $79.9(\mathrm{C}), 55.8\left(\mathrm{CH}_{3}\right), 28.2\left(\mathrm{CH}_{3}\right), 21.7\left(\mathrm{CH}_{3}\right)$; Elemental analysis calcd for $\mathrm{C}_{20} \mathrm{H}_{21} \mathrm{BrO}_{4}$ : C (59.27\%), $\mathrm{H}(5.22 \%)$; found $\mathrm{C}(59.30 \%), \mathrm{H}(5.44 \%)$.

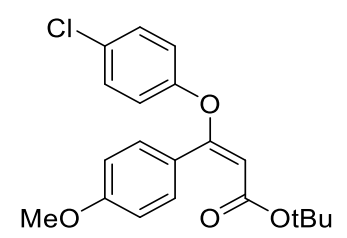

tert-Butyl (E)-3-(4-chlorophenoxy)-3-(4-methoxyphenyl)acrylate ((E)-7). Following Method C, a mixture of tert-butyl 3-(4-methoxyphenyl)propiolate $(0.214 \mathrm{~g}, 0.92 \mathrm{mmol})$, and 4-chlorophenol $(0.130 \mathrm{~g}, 1.012 \mathrm{mmol})$ and potassium carbonate $(0.127 \mathrm{~g}, 0.920 \mathrm{mmol})$ in DMSO $(1.0 \mathrm{~mL})$ was stirred at $60^{\circ} \mathrm{C}$ for $4 \mathrm{~h}$. Crude HPLC analysis showed $\mathrm{E} / \mathrm{Z}$ ratio $=$ 23:77. Following Method D, the crude oil and [Ir(ppy $\left.)_{2}(\mathrm{dtbbpy})\right] \mathrm{PF}_{6}(0.42 \mathrm{mg}, 0.46 \mu \mathrm{mol})$ were stirred in DMSO $(4.6 \mathrm{~mL})$ under laser irradiation for $7 \mathrm{~h}$. The crude product was purified via flash column chromatography ( $20 \% \mathrm{EtOAc/heptanes)} \mathrm{to} \mathrm{provide} \mathrm{the} \mathrm{title}$ compound as a white solid $(0.290 \mathrm{~g}, 87 \%, 92: 8 \mathrm{E} / \mathrm{Z}$ ratio). Recrystallized from heptanes (3 
$\mathrm{mL})$ to afford pure $\mathrm{E}$ isomer as a white solid (188 mg, 59\% yield). $\mathrm{mp} 90.7-92.0{ }^{\circ} \mathrm{C} ;{ }^{1} \mathrm{H}$ NMR (400 MHz, Chloroform- $d$ ) $\delta 7.58-7.50(\mathrm{~m}, 2 \mathrm{H}), 7.38-7.30$ (m, 2H), $7.08-7.00$ $(\mathrm{m}, 2 \mathrm{H}), 6.96-6.87(\mathrm{~m}, 2 \mathrm{H}), 5.10(\mathrm{~s}, 1 \mathrm{H}), 3.84(\mathrm{~s}, 3 \mathrm{H}), 1.35(\mathrm{~s}, 9 \mathrm{H}) ;{ }^{13} \mathrm{C}$ NMR $(101 \mathrm{MHz}$, Chloroform- $d$ ) $\delta 168.2(\mathrm{C}), 166.1(\mathrm{C}), 161.2(\mathrm{C}), 153.2(\mathrm{C}), 131.1(\mathrm{CH}), 130.4(\mathrm{C}), 130.1$ $(\mathrm{CH}), 125.9(\mathrm{C}), 122.6(\mathrm{CH}), 113.4(\mathrm{CH}), 100.9(\mathrm{CH}), 80.2(\mathrm{C}), 55.5\left(\mathrm{CH}_{3}\right), 28.2\left(\mathrm{CH}_{3}\right)$; HRMS (TOF) $m / z:[\mathrm{M}+\mathrm{H}]^{+}$calcd for $\mathrm{C}_{20} \mathrm{H}_{22} \mathrm{ClO}_{4} 361.1200$; found 361.1196.

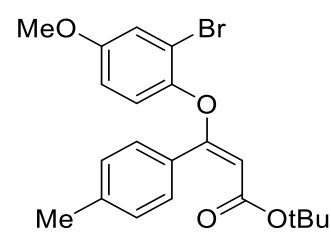

tert-Butyl (E)-3-(2-bromo-4-methoxyphenoxy)-3-(p-tolyl)acrylate $((E)-8)$. Following Method C, a mixture of tert-butyl 3-(p-tolyl)propiolate $(0.433 \mathrm{~g}, 2.00 \mathrm{mmol})$ and 2-bromo4-methoxyphenol $(0.426 \mathrm{~g}, 2.10 \mathrm{mmol})$ and potassium carbonate $(0.276 \mathrm{~g}, 2.00 \mathrm{mmol})$ in DMSO $(2.000 \mathrm{~mL})$ was stirred at $60{ }^{\circ} \mathrm{C}$ for $2 \mathrm{~h}$. Crude HPLC analysis showed E/Z ratio = 28:72. Following Method D, the crude oil and $\left[\operatorname{Ir}(\mathrm{ppy})_{2}(\mathrm{dtbbpy})\right] \mathrm{PF}_{6}(0.91 \mathrm{mg}, 1.0 \mu \mathrm{mol})$ were stirred in DMSO $(13.0 \mathrm{~mL})$ under laser irradiation for $4 \mathrm{~h}$. The crude product was purified via flash column chromatography (20\% EtOAc/heptanes) to provide the title compound as a white solid (633 mg, 75\%, 99:1 E/Z ratio). Recrystallized from heptanes (6 $\mathrm{mL}$ ) to afford pure $\mathrm{E}$ isomer as a white solid (584 mg, 69\% yield). $\mathrm{mp} 102.7-103.4{ }^{\circ} \mathrm{C} ;{ }^{1} \mathrm{H}$ NMR (700 MHz, Chloroform- $d$ ) $\delta 7.58-7.54(\mathrm{~m}, 2 \mathrm{H}), 7.25-7.20(\mathrm{~m}, 2 \mathrm{H}), 7.16(\mathrm{~d}, J=$ $3.0 \mathrm{~Hz}, 1 \mathrm{H}), 7.08(\mathrm{~d}, J=8.9 \mathrm{~Hz}, 1 \mathrm{H}), 6.88(\mathrm{dd}, J=8.9,3.0 \mathrm{~Hz}, 1 \mathrm{H}), 4.91(\mathrm{~s}, 1 \mathrm{H}), 3.81(\mathrm{~s}$, 3H), $2.39(\mathrm{~s}, 3 \mathrm{H}), 1.30(\mathrm{~s}, 9 \mathrm{H}) ;{ }^{13} \mathrm{C}$ NMR (101 MHz, Chloroform- $\left.d\right) \delta 170.0(\mathrm{C}), 166.4$ (C), $157.1(\mathrm{C}), 147.7(\mathrm{C}), 140.1(\mathrm{C}), 131.6(\mathrm{C}), 129.3(\mathrm{CH}), 128.6(\mathrm{CH}), 122.5(\mathrm{CH}), 115.1$ $(\mathrm{CH}), 99.4(\mathrm{CH}), 79.9(\mathrm{C}), 55.8\left(\mathrm{CH}_{3}\right), 28.2\left(\mathrm{CH}_{3}\right), 21.7\left(\mathrm{CH}_{3}\right)$; Elemental analysis calcd for $\mathrm{C}_{21} \mathrm{H}_{23} \mathrm{BrO}_{4}$ : $\mathrm{C}(60.15 \%), \mathrm{H}(5.53 \%)$; found $\mathrm{C}(60.19 \%), \mathrm{H}(5.85 \%)$.

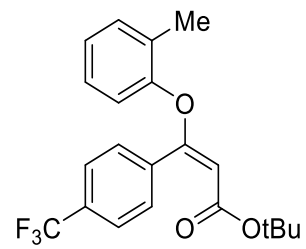

tert-Butyl (E)-3-(o-tolyloxy)-3-(4-(trifluoromethyl)phenyl)acrylate ((E)-9). Following Method C, a mixture of tert-butyl 3-(4-(trifluoromethyl)phenyl)propiolate $(0.2202 \mathrm{~g}, 0.815$ $\mathrm{mmol}), o$-cresol $(0.093 \mathrm{~g}, 0.856 \mathrm{mmol})$, potassium carbonate $(0.011 \mathrm{~g}, 0.081 \mathrm{mmol})$ in DMSO $(0.815 \mathrm{~mL})$ was stirred at $60{ }^{\circ} \mathrm{C}$ for $16 \mathrm{~h}$. Crude HPLC analysis showed E/Z ratio $=22: 78$. Following Method D, the crude oil and $\left[\operatorname{Ir}(\text { ppy })_{2}(\mathrm{dtbbpy})\right] \mathrm{PF}_{6}(0.37 \mathrm{mg}, 0.41$ $\mu \mathrm{mol})$ were stirred in DMSO $(5.4 \mathrm{~mL})$ under laser irradiation for $17 \mathrm{~h}$. The crude product was purified via flash column chromatography (20\% EtOAc/heptanes) to provide the title compound as a white solid $(0.249 \mathrm{~g}, 81 \%, 98: 2 \mathrm{E} / \mathrm{Z}$ ratio $)$. Recrystallization from pet ether $(2 \mathrm{~mL})$ at $0{ }^{\circ} \mathrm{C}$ afforded pure $\mathrm{E}$ isomer as a white solid (187 $\mathrm{mg}, 61 \%$ yield). $\mathrm{mp} 70.8-71.3$ ${ }^{\circ} \mathrm{C} ;{ }^{1} \mathrm{H}$ NMR (400 MHz, Chloroform- $d$ ) $\delta 7.77-7.66(\mathrm{~m}, 4 \mathrm{H}), 7.32-7.22(\mathrm{~m}, 2 \mathrm{H}), 7.18$ $(\mathrm{t}, J=7.2 \mathrm{~Hz}, 1 \mathrm{H}), 7.07(\mathrm{~d}, J=7.9 \mathrm{~Hz}, 1 \mathrm{H}), 4.97(\mathrm{~s}, 1 \mathrm{H}), 2.28(\mathrm{~s}, 3 \mathrm{H}), 1.27(\mathrm{~s}, 9 \mathrm{H}) .{ }^{13} \mathrm{C}$ NMR (176 MHz, Chloroform- $d$ ) $\delta 166.9(\mathrm{C}), 165.7$ (C), $151.8(\mathrm{C}), 138.4\left(\mathrm{~d}, J_{\mathrm{C}-\mathrm{F}}=1.3 \mathrm{~Hz}\right.$, $\mathrm{CH}), 132.0(\mathrm{CH}), 131.7\left(\mathrm{q}, J_{\mathrm{C}-\mathrm{F}}=32.6, \mathrm{C}\right), 130.5(\mathrm{C}), 129.6(\mathrm{CH}), 127.8(\mathrm{CH}), 126.2(\mathrm{CH})$, $125.0\left(\mathrm{q}, J_{\mathrm{C}-\mathrm{F}}=3.7 \mathrm{~Hz}, \mathrm{CH}\right), 123.3\left(\mathrm{~d}, J_{\mathrm{C}-\mathrm{F}}=271.9 \mathrm{~Hz}, \mathrm{C}\right), 121.8(\mathrm{CH}), 99.8(\mathrm{CH}), 80.4$ 
(C), $28.1\left(\mathrm{CH}_{3}\right), 16.2\left(\mathrm{CH}_{3}\right) .{ }^{19} \mathrm{~F}$ NMR (376 MHz, Chloroform- $d$ ) $\delta$-63.7; HRMS (TOF) $m / z:[\mathrm{M}+\mathrm{Na}]^{+}$calcd for $\mathrm{C}_{21} \mathrm{H}_{21} \mathrm{~F}_{3} \mathrm{O}_{3} \mathrm{Na}$ 401.1340; found 401.1316 .<smiles>COC(=O)Oc1cccc(-c2ccc(C(=O)OC)cc2)c1</smiles>

(E)-methyl 4-(3-(tert-butoxy)-1-(3-(difluoromethoxy)phenoxy)-3-oxoprop-1-en-1yl)benzoate $((E)-10)$. Following Method C, a mixture of methyl 4-(3-(tert-butoxy)-3oxoprop-1-yn-1-yl)benzoate $(0.260 \mathrm{~g}, 1.00 \mathrm{mmol})$, and 3-(difluoromethoxy)phenol (0.168 $\mathrm{g}, 1.05 \mathrm{mmol}$, used as $30 \mathrm{wt} \%$ solution in DMSO) and potassium carbonate $(0.014 \mathrm{~g}, 0.10$ mmol) in DMSO $(1.00 \mathrm{~mL})$ was stirred at $60{ }^{\circ} \mathrm{C}$ for $16 \mathrm{~h}$. Crude HPLC analysis showed $\mathrm{E} / \mathrm{Z}$ ratio $=18: 82$. Following Method $\mathrm{D}$, the crude oil and $\left[\operatorname{Ir}(\mathrm{ppy})_{2}(\mathrm{dtbbpy})\right] \mathrm{PF}_{6}(0.46 \mathrm{mg}$, $0.5 \mu \mathrm{mol})$ were stirred in DMSO $(4.00 \mathrm{~mL})$ under laser irradiation for $4 \mathrm{~h}$. The crude product was purified via flash column chromatography (20\% EtOAc/heptanes) to provide the title compound as a colorless oil (332 mg, 79\%, 95:5 E/Z ratio). Recrystallized from heptanes $(2 \mathrm{~mL})$ to afford pure $\mathrm{E}$ isomer as a white solid $(241 \mathrm{mg}$, 57\% yield). $\mathrm{mp} 68.7-$ $71.1{ }^{\circ} \mathrm{C}$; ${ }^{1} \mathrm{H}$ NMR $(700 \mathrm{MHz}$, Chloroform- $d$ ) $\delta 8.10-8.06(\mathrm{~m}, 2 \mathrm{H}), 7.65-7.61(\mathrm{~m}, 2 \mathrm{H})$, $7.38(\mathrm{t}, J=8.2 \mathrm{~Hz}, 1 \mathrm{H}), 7.00-6.96(\mathrm{~m}, 2 \mathrm{H}), 6.90(\mathrm{t}, J=2.3 \mathrm{~Hz}, 1 \mathrm{H}), 6.52(\mathrm{t}, J=73.4 \mathrm{~Hz}$, $1 \mathrm{H}), 5.26(\mathrm{~s}, 1 \mathrm{H}), 3.93(\mathrm{~s}, 3 \mathrm{H}), 1.30(\mathrm{~s}, 9 \mathrm{H}) ;{ }^{13} \mathrm{C}$ NMR $(101 \mathrm{MHz}$, Chloroform- $d) \delta 166.7$ (C), $165.4(\mathrm{C}), 155.0(\mathrm{C}), 152.3$ (t, $J=2.9 \mathrm{~Hz}, \mathrm{C}), 138.4(\mathrm{C}), 131.4(\mathrm{C}), 131.0(\mathrm{CH}), 129.5$ $(\mathrm{CH}), 129.3(\mathrm{CH}), 118.1(\mathrm{CH}), 116.3(\mathrm{CH}), 115.8(\mathrm{t}, J=261.7,260.3 \mathrm{~Hz}, \mathrm{CH}), 112.9(\mathrm{CH})$, $103.1(\mathrm{CH}), 80.8(\mathrm{C}), 52.4\left(\mathrm{CH}_{3}\right), 28.1\left(\mathrm{CH}_{3}\right)$.; ${ }^{19} \mathrm{~F}$ NMR $(376 \mathrm{MHz}$, Chloroform- $d$ ) $\delta$ 82.15; HRMS (TOF) $m / z$ : [M + Na] $]^{+}$calcd for $\mathrm{C}_{22} \mathrm{H}_{22} \mathrm{~F}_{2} \mathrm{O}_{6} \mathrm{Na} 443.1282$; found 443.1254.

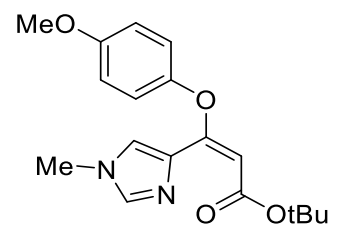

tert-Butyl (E)-3-(4-methoxyphenoxy)-3-(1-methyl-1H-imidazol-4-yl)acrylate ((E)-11). Following Method C, a mixture of tert-butyl 3-(1-methyl-1H-imidazol-5-yl)propiolate $(0.206 \mathrm{~g}, 1.00 \mathrm{mmol})$ and 4-methoxyphenol $(0.130 \mathrm{~g}, 1.05 \mathrm{mmol})$ and potassium carbonate $(0.014 \mathrm{~g}, 0.10 \mathrm{mmol})$ in DMSO $(1.00 \mathrm{~mL})$ was stirred at $60{ }^{\circ} \mathrm{C}$ for $2 \mathrm{~h}$. Crude HPLC analysis showed E/Z ratio $=31: 69$. Following Method D, $0.25 \mathrm{mmol}$ of the crude oil and $\left[\operatorname{Ir}(\mathrm{ppy})_{2}(\mathrm{dtbbpy})\right] \mathrm{PF}_{6}(0.22 \mathrm{mg}, 0.25 \mu \mathrm{mol})$ were stirred in DMSO $(1.25 \mathrm{~mL})$ under laser irradiation for $3 \mathrm{~h}$. The crude product was purified via flash column chromatography (20\% EtOAc/heptanes) to provide the title compound as a white solid (54 mg, 66\%, 94:6 E/Z ratio). Recrystallized from 1:1 MTBE/heptanes $(2 \mathrm{~mL})$ to afford pure E isomer as a white solid (30 mg, 36\% yield). mp 83.3-85.1 ${ }^{\circ} \mathrm{C}$; ${ }^{1} \mathrm{H}$ NMR $(700 \mathrm{MHz}$, Chloroform- $d$ ) $\delta 7.52-$ $7.49(\mathrm{~m}, 1 \mathrm{H}), 7.35(\mathrm{~d}, J=1.1 \mathrm{~Hz}, 1 \mathrm{H}), 7.04-6.99(\mathrm{~m}, 2 \mathrm{H}), 6.94-6.89(\mathrm{~m}, 2 \mathrm{H}), 5.21(\mathrm{~s}$, $1 \mathrm{H}), 3.82(\mathrm{~s}, 3 \mathrm{H}), 3.71(\mathrm{~s}, 3 \mathrm{H}), 1.34(\mathrm{~s}, 9 \mathrm{H}) ;{ }^{13} \mathrm{C}$ NMR $(176 \mathrm{MHz}$, Chloroform- $d$ ) $\delta 165.5$ (C), $158.6(\mathrm{C}), 157.4(\mathrm{C}), 147.0(\mathrm{C}), 139.5(\mathrm{CH}), 133.0(\mathrm{CH}), 126.1(\mathrm{C}), 122.2(\mathrm{CH}), 115.3$ $(\mathrm{CH}), 103.4(\mathrm{CH}), 80.5(\mathrm{C}), 55.8\left(\mathrm{CH}_{3}\right), 32.7\left(\mathrm{CH}_{3}\right), 28.2\left(\mathrm{CH}_{3}\right)$; HRMS (TOF) $m / z:[\mathrm{M}+$ $\mathrm{H}]^{+}$calcd for $\mathrm{C}_{18} \mathrm{H}_{23} \mathrm{~N}_{2} \mathrm{O}_{4}$ 331.1652; found 331.1663. 


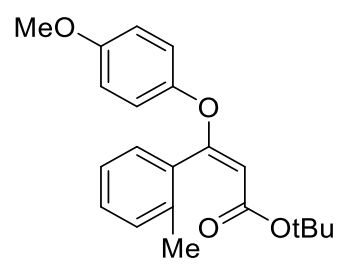

tert-Butyl (E)-3-(4-methoxyphenoxy)-3-(o-tolyl)acrylate ((E)-12). Following Method C, a mixture of tert-butyl 3-(o-tolyl)propiolate $(0.216 \mathrm{~g}, 1.00 \mathrm{mmol})$, 4-methoxyphenol $(0.130 \mathrm{~g}, 1.050 \mathrm{mmol})$, potassium carbonate $(0.138 \mathrm{~g}, 1.00 \mathrm{mmol})$ in DMSO $(1.0 \mathrm{~mL})$ was stirred at $60{ }^{\circ} \mathrm{C}$ for $24 \mathrm{~h}$. Crude HPLC analysis showed $\mathrm{E} / \mathrm{Z}$ ratio $=53: 47$. Following Method D with the exception of using $\operatorname{Ir}(\mathrm{ppy})_{3}(0.45 \mathrm{mg}, 0.5 \mu \mathrm{mol})$, the crude oil and catalyst were stirred in DMSO $(5.0 \mathrm{~mL})$ under laser irradiation for $3 \mathrm{~h}$. The crude product was purified via flash column chromatography (20\% EtOAc/heptanes) to provide the title compound as a white solid $(0.279 \mathrm{~g}, 82 \%, 97: 3 \mathrm{E} / \mathrm{Z}$ ratio). The crude solid was slurried in pentane $(3 \times 1 \mathrm{~mL})$ to afford pure $\mathrm{E}$ isomer as a white solid $(247 \mathrm{mg}, 73 \%$ yield). $\mathrm{mp}$ 73.7-74.4 ${ }^{\circ} \mathrm{C} ;{ }^{1} \mathrm{H}$ NMR (700 MHz, Chloroform- $d$ ) $\delta 7.32-7.29(\mathrm{~m}, 2 \mathrm{H}), 7.26-7.21(\mathrm{~m}$, $2 \mathrm{H}), 7.09-7.04(\mathrm{~m}, 2 \mathrm{H}), 6.95-6.90(\mathrm{~m}, 2 \mathrm{H}), 5.09(\mathrm{~s}, 1 \mathrm{H}), 3.82(\mathrm{~s}, 3 \mathrm{H}), 2.44(\mathrm{~s}, 3 \mathrm{H})$, 1.17 (s, 9H); ${ }^{13} \mathrm{C}$ NMR (176 MHz, Chloroform- $d$ ) $\delta 170.2$ (C), $166.0(\mathrm{C}), 157.3(\mathrm{C})$, $147.2(\mathrm{C}), 136.1(\mathrm{C}), 135.4(\mathrm{C}), 130.0(\mathrm{CH}), 129.3(\mathrm{CH}), 129.0(\mathrm{CH}), 125.5(\mathrm{CH}), 122.6$ $(\mathrm{CH}), 115.1(\mathrm{CH}), 100.7(\mathrm{CH}), 79.7(\mathrm{C}), 55.8\left(\mathrm{CH}_{3}\right), 27.9\left(\mathrm{CH}_{3}\right), 19.5\left(\mathrm{CH}_{3}\right)$; HRMS (TOF) $m / z:[\mathrm{M}+\mathrm{H}]^{+}$calcd for $\mathrm{C}_{21} \mathrm{H}_{25} \mathrm{O}_{4} 341.1747$; found 341.1755 .

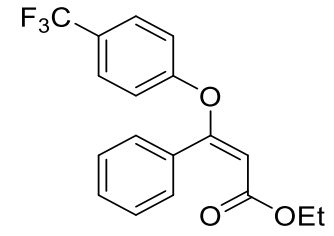

Ethyl (E)-3-(4-methoxyphenoxy)-3-phenylacrylate $((E)-13)$. Following Method C, a mixture of ethyl 3-phenylpropiolate $(0.174 \mathrm{~g}, 1.00 \mathrm{mmol})$, and 4-(trifluoromethyl)phenol $(0.170 \mathrm{~g}, 1.05 \mathrm{mmol})$ and potassium carbonate $(0.014 \mathrm{~g}, 0.10 \mathrm{mmol})$ in DMSO $(1.00 \mathrm{~mL})$ was stirred at $60{ }^{\circ} \mathrm{C}$ for $16 \mathrm{~h}$. Crude HPLC analysis showed E/Z ratio $=28: 72$. Following Method D, the crude oil and $\left[\operatorname{Ir}(\text { ppy })_{2}(\mathrm{dtbbpy})\right] \mathrm{PF}_{6}(0.91 \mathrm{mg}, 1.0 \mu \mathrm{mol})$ were stirred in $\mathrm{MeCN}(5.00 \mathrm{~mL})$ under laser irradiation for $3 \mathrm{~h}$. The crude product was purified via flash column chromatography (20\% EtOAc/heptanes) to provide the title compound as a colorless oil (306 mg, 91\%, 91:9 E/Z ratio). Major: ${ }^{1} \mathrm{H}$ NMR (400 MHz, Chloroform-d) $\delta$ $7.69-7.57(\mathrm{~m}, 4 \mathrm{H}), 7.54-7.33(\mathrm{~m}, 3 \mathrm{H}), 7.28-7.19(\mathrm{~m}, 2 \mathrm{H}), 5.30(\mathrm{~s}, 1 \mathrm{H}), 4.05(\mathrm{q}, J=$ $7.1 \mathrm{~Hz}, 1 \mathrm{H}), 1.14(\mathrm{t}, J=7.1 \mathrm{~Hz}, 3 \mathrm{H}) ;{ }^{13} \mathrm{C}$ NMR $(101 \mathrm{MHz}$, Chloroform- $d) \delta 168.7$ (C), $166.2(\mathrm{C}), 157.1(\mathrm{~d}, J=1.5 \mathrm{~Hz}, \mathrm{CH}), 133.2(\mathrm{C}), 130.6(\mathrm{CH}), 129.5(\mathrm{CH}), 128.1(\mathrm{CH}), 127.6$ $\left(\mathrm{q}, J_{\mathrm{C}-\mathrm{F}}=3.8 \mathrm{~Hz}, \mathrm{CH}\right), 127.5\left(\mathrm{q}, J_{\mathrm{C}-\mathrm{F}}=36.2 \mathrm{~Hz}, \mathrm{C}\right), 124.0\left(\mathrm{q}, J_{\mathrm{C}-\mathrm{F}}=271.6 \mathrm{~Hz}, \mathrm{C}\right), 121.3$ $(\mathrm{CH}), 100.9(\mathrm{CH}), 60.3\left(\mathrm{CH}_{2}\right), 14.2\left(\mathrm{CH}_{3}\right) ;{ }^{19} \mathrm{~F}$ NMR $(376 \mathrm{MHz}$, Chloroform- $d) \delta$-63.0; HRMS (TOF) $m / z:[\mathrm{M}+\mathrm{H}]^{+}$calcd for $\mathrm{C}_{18} \mathrm{H}_{16} \mathrm{~F}_{3} \mathrm{O}_{3}$ 337.1045; found 337.1043. 


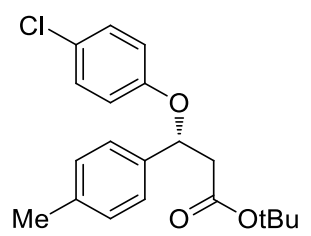

(R)-tert-butyl 3-(4-chlorophenoxy)-3-(p-tolyl)propanoate (15). Using Method E, the following amounts of regents were used, bis(norbornadiene)rhodium(I) tetrafluoroborate $(0.26 \mathrm{mg}, \quad 0.70 \mu \mathrm{mol})$ and J505-1 $(0.44 \mathrm{mg}, 0.77 \mu \mathrm{mol})$, tert-Butyl (E)-3-(4chlorophenoxy)-3-(p-tolyl)acrylate $(0.16 \mathrm{~g}, 0.46 \mathrm{mmol})$, and $\mathrm{CH}_{2} \mathrm{Cl}_{2}(0.95 \mathrm{~mL})$. After 72 $\mathrm{h}$, the reaction was complete and the crude product was purified by column chromatography, using ethyl acetate/heptane as eluent to afford the title compound $(0.15$ g, 91\%) as a clear oil. ${ }^{1} \mathrm{H}$ NMR (400 MHz, Chloroform-d) $\delta 7.26-7.20(\mathrm{~m}, 2 \mathrm{H}), 7.15-$ 7.07 (m, 4H), $6.79-6.74(\mathrm{~m}, 2 \mathrm{H}), 5.47$ (dd, $J=9.3,4.5 \mathrm{~Hz}, 1 \mathrm{H}), 2.89$ (dd, $J=15.4,9.3$ $\mathrm{Hz}, 1 \mathrm{H}), 2.65(\mathrm{dd}, J=15.4,4.5 \mathrm{~Hz}, 1 \mathrm{H}), 2.30(\mathrm{~s}, 3 \mathrm{H}), 1.40(\mathrm{~s}, 9 \mathrm{H}) .{ }^{13} \mathrm{C}$ NMR $(101 \mathrm{MHz}$, Chloroform- $d$ ) $\delta 169.7(\mathrm{C}), 156.7(\mathrm{C}), 137.9(\mathrm{C}), 137.2(\mathrm{C}), 129.6(\mathrm{C}), 129.4(\mathrm{CH}), 129.3$ $(\mathrm{CH}), 126.1(\mathrm{C}), 126.0(\mathrm{CH}), 117.6(\mathrm{CH}), 81.2(\mathrm{C}), 77.7\left(\mathrm{CH}_{3}\right), 45.2\left(\mathrm{CH}_{2}\right), 28.2\left(\mathrm{CH}_{3}\right)$, $21.2\left(\mathrm{CH}_{3}\right)$. HRMS (FTMS) $m / z:[\mathrm{M}+\mathrm{Na}]^{+}$calcd for $\mathrm{C}_{20} \mathrm{H}_{23} \mathrm{ClO}_{3} \mathrm{Na} 369.1228$; found 369.1218. $[\alpha]_{\mathrm{D}}{ }^{23}+30.2\left(c 0.64, \mathrm{CHCl}_{3}\right)$; HPLC analysis $((\mathrm{R}, \mathrm{R})-$ Whelk-O 1 , part number 780222; 97\% hexanes, 3\% (9:1) hexanes:EtOH (abs), $1.0 \mathrm{~mL} / \mathrm{min}$, isocratic, $230 \mathrm{~nm})$ indicated $91 \%$ ee: $t_{R}($ major $)=8.1 \mathrm{~min}, t_{R}($ minor $)=9.6 \mathrm{~min}$

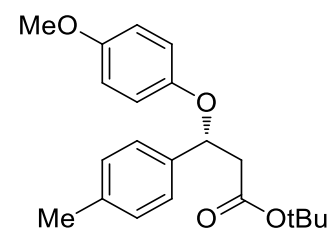

(R)-tert-butyl 3-(4-methoxyphenoxy)-3-(p-tolyl)propanoate (16). Using Method E, the following amounts of regents were used, bis(norbornadiene)rhodium(I) tetrafluoroborate $(0.19 \mathrm{mg}, \quad 0.51 \mu \mathrm{mol})$ and J505-1 $(0.32 \mathrm{mg}, 0.57 \mu \mathrm{mol})$, tert-Butyl (E)-3-(4methoxyphenoxy)-3-(p-tolyl)acrylate $(0.18 \mathrm{~g}, 0.51 \mathrm{mmol})$, and $\mathrm{CH}_{2} \mathrm{Cl}_{2}(1.0 \mathrm{~mL})$. After 4 $\mathrm{h}$, the reaction was complete and the crude product was purified by column chromatography, using ethyl acetate/heptane as eluent to afford the title compound $(0.15$ g, 86\%) as a clear oil. ${ }^{1} \mathrm{H}$ NMR (400 MHz, Chloroform- $d$ ) $\delta 7.27-7.25(\mathrm{~m}, 2 \mathrm{H}), 7.13(\mathrm{~d}, J$ $=7.6 \mathrm{~Hz}, 2 \mathrm{H}), 6.79-6.76(\mathrm{~m}, 2 \mathrm{H}), 6.73-6.69(\mathrm{~m}, 2 \mathrm{H}), 5.41(\mathrm{dd}, J=9.0,4.4 \mathrm{~Hz}, 1 \mathrm{H}), 3.70$ (s, 3H), 2.89 (dd, $J=15.4,9.2 \mathrm{~Hz}, 1 \mathrm{H}), 2.65(\mathrm{dd}, J=15.0,4.4 \mathrm{~Hz}, 1 \mathrm{H}), 2.31(\mathrm{~s}, 3 \mathrm{H}), 1.41$ (s, 9H). ${ }^{13} \mathrm{C}$ NMR (101 MHz, Chloroform- $d$ ) $\delta 170.0(\mathrm{C}), 154.1(\mathrm{C}), 152.3(\mathrm{C}), 137.9(\mathrm{C})$, $137.7(\mathrm{C}), 129.4(\mathrm{CH}), 126.2(\mathrm{CH}), 117.6(\mathrm{CH}), 114.5(\mathrm{CH}), 81.0(\mathrm{C}), 78.3\left(\mathrm{CH}_{3}\right), 55.7$ $(\mathrm{CH}), 45.2\left(\mathrm{CH}_{2}\right), 28.2\left(\mathrm{CH}_{3}\right), 21.3\left(\mathrm{CH}_{3}\right)$. Elemental analysis Cald. for $\mathrm{C}_{12} \mathrm{H}_{26} \mathrm{O}_{4}$ : $\mathrm{C}$ (73.66\%), H (7.65\%); found C (73.87\%), H (7.79\%); $[\alpha]_{\mathrm{D}}^{23}+46.1\left(c 1.00, \mathrm{CHCl}_{3}\right)$; HPLC analysis ((R,R)-Whelk-O 1, part number 780222; $90 \%$ hexanes, 10\% (9:1) hexanes:EtOH (abs), isocratic, $1.0 \mathrm{~mL} / \mathrm{min}, 230 \mathrm{~nm})$ indicated $93 \%$ ee: $\mathrm{t}_{\mathrm{R}}($ major $)=9.5 \mathrm{~min}, \mathrm{t}_{\mathrm{R}}($ minor $)=$ $10.5 \mathrm{~min}$. 


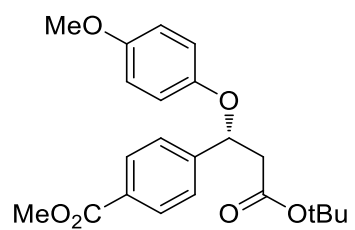

(R)-methyl 4-(3-(tert-butoxy)-1-(4-methoxyphenoxy)-3-oxopropyl)benzoate (17). Using Method E, the following amounts of regents were used, bis(norbornadiene)rhodium(I) tetrafluoroborate $(0.086 \mathrm{mg}, 0.23 \mu \mathrm{mol})$ and J505-1 $(0.14 \mathrm{mg}, 0.25 \mu \mathrm{mol})$, methyl (E)-4(3-(tert-butoxy)-1-(4-methoxyphenoxy)-3-oxoprop-1-en-1-yl)benzoate $\quad(0.18 \mathrm{~g}, \quad 0.46$ $\mathrm{mmol})$, and $\mathrm{CH}_{2} \mathrm{Cl}_{2}(1.0 \mathrm{~mL})$. After $2 \mathrm{~h}$, the reaction was complete and the crude product was purified by column chromatography, using ethyl acetate/heptane as eluent to afford the title compound $(0.16 \mathrm{~g}, 91 \%)$ as a clear oil. ${ }^{1} \mathrm{H}$ NMR $(400 \mathrm{MHz}$, Chloroform- $d$ ) $\delta 8.02$ $7.98(\mathrm{~m}, 2 \mathrm{H}), 7.48-7.44(\mathrm{~m}, 2 \mathrm{H}), 6.78-6.75(\mathrm{~m}, 2 \mathrm{H}), 6.73-6.70(\mathrm{~m}, 2 \mathrm{H}), 5.50(\mathrm{dd}, J=8.8$, $4.8 \mathrm{~Hz}, 1 \mathrm{H}), 3.90(\mathrm{~s}, 3 \mathrm{H}), 3.70(\mathrm{~s}, 3 \mathrm{H}), 2.91(\mathrm{dd}, J=15.6,8.8 \mathrm{~Hz}, 1 \mathrm{H}), 2.67$ (dd, $J=15.2$, $4.8 \mathrm{~Hz}, 1 \mathrm{H}), 1.41(\mathrm{~s}, 9 \mathrm{H}) .{ }^{13} \mathrm{C}$ NMR (101 MHz, Chloroform-d) $\delta 169.5(\mathrm{C}), 166.9(\mathrm{C})$, $154.4(\mathrm{C}), 151.9(\mathrm{C}), 146.1(\mathrm{C}), 130.1(\mathrm{CH}), 129.9(\mathrm{C}), 126.4(\mathrm{CH}), 117.6(\mathrm{CH}), 114.6$ $(\mathrm{CH}), 81.3(\mathrm{C}), 77.9\left(\mathrm{CH}_{3}\right), 55.7(\mathrm{CH}), 52.2\left(\mathrm{CH}_{3}\right), 44.8\left(\mathrm{CH}_{2}\right), 28.2\left(\mathrm{CH}_{3}\right)$. Elemental analysis Cald. for $\mathrm{C}_{22} \mathrm{H}_{26} \mathrm{O}_{6}: \mathrm{C}(68.38 \%), \mathrm{H}(6.78 \%)$; found $\mathrm{C}(68.54 \%), \mathrm{H}(7.14 \%)$. [ $\left.\alpha\right]_{\mathrm{D}}{ }^{23}$ +47.4 ( $c$ 0.49, $\mathrm{CHCl}_{3}$ ) HPLC analysis (ChiralPak IC, part number 83325; $90 \%$ hexanes, $10 \%(9: 1)$ hexanes:EtOH (abs), isocratic, $1.0 \mathrm{~mL} / \mathrm{min}, 210 \mathrm{~nm})$ indicated $92 \%$ ee: $\mathrm{t}_{\mathrm{R}}$ $($ major $)=21.2 \mathrm{~min}, \mathrm{t}_{\mathrm{R}}($ minor $)=25.6 \mathrm{~min}$.

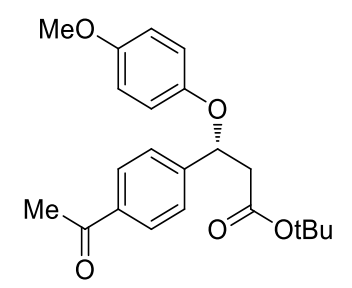

(R)-tert-butyl 3-(4-acetylphenyl)-3-(4-methoxyphenoxy)propanoate (18): Using Method $\mathrm{E}$, the following amounts of regents were used, bis(norbornadiene)rhodium(I) tetrafluoroborate $(0.86 \mathrm{mg}, 2.29 \mu \mathrm{mol})$ and J505-1 (1.44 mg, $2.52 \mu \mathrm{mol})$, methyl (E)-4-(3(tert-butoxy)-1-(4-(methoxycarbonyl)phenoxy)-3-oxoprop-1-en-1-yl)benzoate $(0.17 \mathrm{~g}$, $0.46 \mathrm{mmol})$, and THF $(1.0 \mathrm{~mL})$. After $72 \mathrm{~h}$, the reaction was complete and the crude product was purified by column chromatography, using ethyl acetate/heptane as eluent to afford the title compound $(0.15 \mathrm{~g}, 92 \%)$ as a clear oil. ${ }^{1} \mathrm{H}$ NMR $(400 \mathrm{MHz}$, Chloroform- $d$ ) $\delta 8.00-7.89(\mathrm{~m}, 2 \mathrm{H}), 7.56-7.46(\mathrm{~m}, 2 \mathrm{H}), 6.83-6.77(\mathrm{~m}, 2 \mathrm{H}), 6.74(\mathrm{~d}, J=9.2 \mathrm{~Hz}, 2 \mathrm{H})$, $5.53(\mathrm{dd}, J=8.9,4.8 \mathrm{~Hz}, 1 \mathrm{H}), 3.73(\mathrm{~s}, 1 \mathrm{H}), 2.94(\mathrm{dd}, J=15.4,8.9 \mathrm{~Hz}, 1 \mathrm{H}), 2.70(\mathrm{dd}, J=$ 15.4, $4.8 \mathrm{~Hz}, 1 \mathrm{H}), 2.60$ (s, 3H), 1.44 (s, 9H). ${ }^{13} \mathrm{C}$ NMR (101 MHz, Chloroform-d) $\delta 197.7$ (C), $169.5(\mathrm{C}), 154.4(\mathrm{C}), 151.8(\mathrm{C}), 146.3(\mathrm{C}), 136.9(\mathrm{C}), 128.9(\mathrm{CH}), 126.6(\mathrm{CH}), 117.5$ $(\mathrm{CH}), 114.6(\mathrm{CH}), 81.4(\mathrm{C}), 77.9(\mathrm{CH}), 55.7(\mathrm{CH}), 44.8\left(\mathrm{CH}_{2}\right), 28.2\left(\mathrm{CH}_{3}\right), 26.8\left(\mathrm{CH}_{3}\right)$. $[\alpha]_{\mathrm{D}}^{23}+46.4\left(c\right.$ 1.01, $\mathrm{CHCl}_{3}$ ). HRMS (FTMS) $\mathrm{m} / \mathrm{z}:[\mathrm{M}+\mathrm{Na}]^{+}$calcd for $\mathrm{C}_{22} \mathrm{H}_{26} \mathrm{O}_{5} \mathrm{Na}$ 393.1673; found 393.16643. HPLC analysis (ChiralPak IA, part number 80325; $70 \%$ hexanes, $30 \%$ (9:1) hexanes:EtOH (abs), isocratic, $1.0 \mathrm{~mL} / \mathrm{min}, 230 \mathrm{~nm}$ ) indicated $92 \%$ ee: $\mathrm{t}_{\mathrm{R}}($ major $)=12.7 \mathrm{~min}, \mathrm{t}_{\mathrm{R}}($ minor $)=16.8 \mathrm{~min}$. 


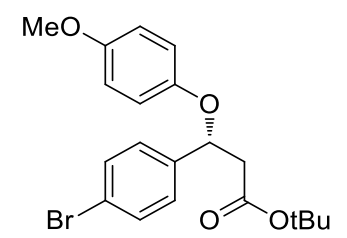

(R)-tert-butyl 3-(4-bromophenyl)-3-(4-methoxyphenoxy)propanoate (19). Using Method $\mathrm{E}$, the following amounts of regents were used, bis(norbornadiene)rhodium(I) tetrafluoroborate $(0.12 \mathrm{mg}, 0.31 \mu \mathrm{mol})$ and J505-1 $(0.19 \mathrm{mg}, 0.34 \mu \mathrm{mol})$, tert-Butyl (E)-3(4-bromophenyl)-3-(4-methoxyphenoxy)acrylate $(0.25 \mathrm{~g}, 0.63 \mathrm{mmol})$, and $\mathrm{CH}_{2} \mathrm{Cl}_{2}(1.25$ $\mathrm{mL}$ ). After $16 \mathrm{~h}$, the reaction was complete and the crude product was purified by column chromatography, using ethyl acetate/heptane as eluent to afford the title compound (0.24 g, 95\%) as a clear oil. ${ }^{1} \mathrm{H}$ NMR $(400 \mathrm{MHz}$, Chloroform- $d$ ) $\delta 7.58-7.37(\mathrm{~m}, 2 \mathrm{H}), 7.34-$ $7.17(\mathrm{~m}, 2 \mathrm{H}), 6.89-6.61(\mathrm{~m}, 2 \mathrm{H}), 5.42(\mathrm{dd}, J=8.9,4.9 \mathrm{~Hz}, 1 \mathrm{H}), 3.71(\mathrm{~s}, 3 \mathrm{H}), 2.90(\mathrm{dd}, J$ $=15.3,8.9 \mathrm{~Hz}, 1 \mathrm{H}), 2.65(\mathrm{dd}, J=15.3,4.9 \mathrm{~Hz}, 1 \mathrm{H}), 1.42(\mathrm{~s}, 9 \mathrm{H}) .{ }^{13} \mathrm{C}$ NMR $(101 \mathrm{MHz}$, Chloroform- $d$ ) $\delta 169.6(\mathrm{C}), 154.4(\mathrm{C}), 151.8(\mathrm{C}), 139.9(\mathrm{C}), 131.9(\mathrm{CH}), 128.1(\mathrm{CH}), 121.9$ $(\mathrm{C}), 117.6(\mathrm{CH}), 114.6(\mathrm{CH}), 81.2(\mathrm{C}), 77.7(\mathrm{CH}), 55.7\left(\mathrm{CH}_{3}\right), 44.9\left(\mathrm{CH}_{2}\right), 28.2\left(\mathrm{CH}_{3}\right)$. $[\alpha]_{\mathrm{D}}^{23}+50.4\left(c\right.$ 1.57, $\left.\mathrm{CHCl}_{3}\right)$ HRMS (FTMS) $m / z:[\mathrm{M}+\mathrm{Na}]^{+}$calcd for $\mathrm{C}_{20} \mathrm{H}_{23} \mathrm{BrO}_{4} \mathrm{Na}$ 429.0672; found 429.0663. HPLC analysis ((R,R)-Whelk-O 1, part number 780222; 85\% hexanes, $15 \%$ (9:1) hexanes: EtOH (abs), isocratic, $1.0 \mathrm{~mL} / \mathrm{min}, 230 \mathrm{~nm}$ ) indicated $95 \%$ ee: $\mathrm{tR}($ major $)=9.1 \mathrm{~min}, \mathrm{tR}($ minor $)=10.2 \mathrm{~min}$.

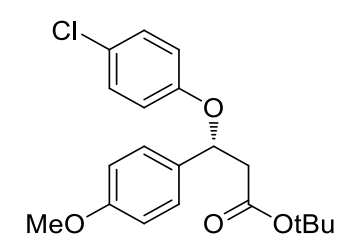

(R)-tert-butyl 3-(4-chlorophenoxy)-3-(4-methoxyphenyl)propanoate (20). Using Method $\mathrm{E}$, the following amounts of regents were used, bis(norbornadiene)rhodium(I) tetrafluoroborate $(0.078 \mathrm{mg}, 0.21 \mu \mathrm{mol})$ and J505-1 $(0.13 \mathrm{mg}, 0.23 \mu \mathrm{mol})$, tert-Butyl (E)3-(4-chlorophenoxy)-3-(4-methoxyphenyl)acrylate (0.15 g, $0.42 \mathrm{mmol})$, and $\mathrm{CH}_{2} \mathrm{Cl}_{2}(0.95$ $\mathrm{mL}$ ). After $2 \mathrm{~h}$, the reaction was complete and the crude product was purified by column chromatography, using ethyl acetate/heptane as eluent to afford the title compound (0.14 g, 93\%) as a clear oil. ${ }^{1} \mathrm{H}$ NMR $(400 \mathrm{MHz}$, Chloroform- $d) \delta 7.31-7.22(\mathrm{~m}, 2 \mathrm{H}), 7.16-$ $7.06(\mathrm{~m}, 2 \mathrm{H}), 6.90-6.81(\mathrm{~m}, 2 \mathrm{H}), 6.81-6.73(\mathrm{~m}, 2 \mathrm{H}), 5.45(\mathrm{dd}, J=9.0,4.8 \mathrm{~Hz}, 1 \mathrm{H}), 3.77$ (s, 3H), $2.90(\mathrm{dd}, J=8.0,6.4,1 \mathrm{H}), 2.65(\mathrm{dd}, J=9.6,4.8 \mathrm{~Hz}, 1 \mathrm{H}), 1.40(\mathrm{~s}, 9 \mathrm{H}) .{ }^{13} \mathrm{C}$ NMR (101 MHz, Chloroform- $d$ ) $\delta 169.7$ (C), $159.5(\mathrm{C}), 156.7$ (C), $132.1(\mathrm{C}), 129.3(\mathrm{CH}), 127.5$ $(\mathrm{CH}), 126.0(\mathrm{C}), 117.7(\mathrm{CH}), 114.3(\mathrm{CH}), 81.2(\mathrm{C}), 55.4\left(\mathrm{CH}_{3}\right), 45.1\left(\mathrm{CH}_{2}\right), 28.2\left(\mathrm{CH}_{3}\right)$. Elemental analysis Cald. for $\mathrm{C}_{20} \mathrm{H}_{23} \mathrm{ClO}_{4}$ : C (66.20\%), $\mathrm{H}(6.39 \%)$; found $\mathrm{C}(65.84 \%), \mathrm{H}$ $(6.42 \%) \cdot[\alpha]_{\mathrm{D}}^{23}+37.3\left(c 1.07, \mathrm{CHCl}_{3}\right)$. HPLC analysis $((\mathrm{R}, \mathrm{R})-$ Whelk-O 1 , part number 780222; 90\% hexanes, $10 \%$ (9:1) hexanes:EtOH (abs), isocratic, $1.0 \mathrm{~mL} / \mathrm{min}, 230 \mathrm{~nm}$ ) indicated 97\% ee: $\mathrm{t}_{\mathrm{R}}($ major $)=8.2 \mathrm{~min}, \mathrm{t}_{\mathrm{R}}($ minor $)=9.2 \mathrm{~min}$. 


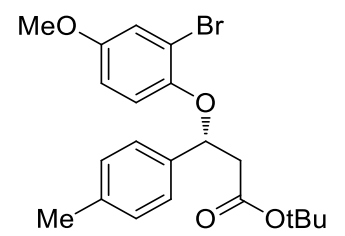

(R)-tert-butyl 3-(2-bromo-4-methoxyphenoxy)-3-(p-tolyl)propanoate (21). Using Method $\mathrm{E}$, the following amounts of regents were used, bis(norbornadiene)rhodium(I) tetrafluoroborate $(0.23 \mathrm{mg}, 0.60 \mu \mathrm{mol})$ and $\mathrm{J} 505-1(0.38 \mathrm{mg}, 0.66 \mu \mathrm{mol})$, tert-Butyl (E)-3(2-bromo-4-methoxyphenoxy)-3-( $p$-tolyl)acrylate $(0.51 \mathrm{~g}, 1.21 \mathrm{mmol})$, and $\mathrm{CH}_{2} \mathrm{Cl}_{2}(2.5$ $\mathrm{mL}$ ). After $16 \mathrm{~h}$, the reaction was complete and the crude product was purified by column chromatography, using ethyl acetate/heptane as eluent to afford the title compound ( 0.45 $\mathrm{g}, 89 \%)$ as a clear oil. ${ }^{1} \mathrm{H}$ NMR $(400 \mathrm{MHz}$, Chloroform- $d$ ) $\delta 7.26(\mathrm{dd}, J=7.9,6.0 \mathrm{~Hz}, 2 \mathrm{H})$, $7.11(\mathrm{~d}, J=7.8 \mathrm{~Hz}, 2 \mathrm{H}), 7.04(\mathrm{~d}, J=2.9 \mathrm{~Hz}, 1 \mathrm{H}), 6.69(\mathrm{~d}, J=9.0 \mathrm{~Hz}, 1 \mathrm{H}), 6.61(\mathrm{dd}, J=$ $9.0,3.0 \mathrm{~Hz}, 1 \mathrm{H}), 5.48(\mathrm{dd}, J=8.7,5.0 \mathrm{~Hz}, 1 \mathrm{H}), 3.67(\mathrm{~s}, 3 \mathrm{H}), 3.00(\mathrm{dd}, J=15.4,8.7 \mathrm{~Hz}$, $1 \mathrm{H}), 2.68$ (dd, $J=15.4,5.0 \mathrm{~Hz}, 1 \mathrm{H}), 2.30$ (s, 3H), 1.39 (s, 9H). ${ }^{13} \mathrm{C}$ NMR $(101 \mathrm{MHz}$, Chloroform- $d$ ) $\delta 169.8(\mathrm{C}), 154.2(\mathrm{C}), 148.7(\mathrm{C}), 137.9(\mathrm{C}), 137.1(\mathrm{C}), 129.4(\mathrm{CH}), 126.4$ $(\mathrm{CH}), 118.8(\mathrm{CH}), 116.4(\mathrm{CH}), 113.7(\mathrm{CH}), 113.3(\mathrm{C}), 81.1(\mathrm{C}), 78.7(\mathrm{CH}), 55.9\left(\mathrm{CH}_{3}\right)$, $44.8\left(\mathrm{CH}_{2}\right), 28.2\left(\mathrm{CH}_{3}\right), 21.3\left(\mathrm{CH}_{3}\right)$. Elemental analysis Cald. for $\mathrm{C}_{21} \mathrm{H}_{25} \mathrm{BrO}_{4}: \mathrm{C}(59.87 \%)$, $\mathrm{H}(5.98 \%)$; found $\mathrm{C}(59.64 \%), \mathrm{H}(5.77 \%)$. $[\alpha]_{\mathrm{D}}^{23}-13.6\left(c 1.48, \mathrm{CHCl}_{3}\right)$. HPLC analysis $((\mathrm{R}, \mathrm{R})-$ Whelk-O 1, part number 780222; 85\% hexanes, $15 \%$ (9:1) hexanes:EtOH (abs), isocratic, $1.0 \mathrm{~mL} / \mathrm{min}, 230 \mathrm{~nm}$ ) indicated $97 \%$ ee: $\mathrm{tR}$ (major) $=8.2 \mathrm{~min}$, $\mathrm{R}($ minor $)=9.5$ $\min$.

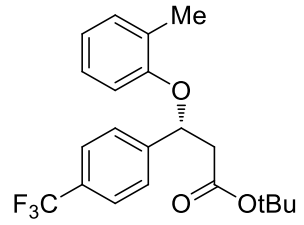

(R)-tert-butyl 3-(o-tolyloxy)-3-(4-(trifluoromethyl)phenyl)propanoate (22). Using Method $\mathrm{E}$, the following amounts of regents were used, bis(norbornadiene)rhodium(I) tetrafluoroborate $(0.80 \mathrm{mg}, 2.14 \mu \mathrm{mol})$ and J505-1 (1.34 mg, $2.35 \mu \mathrm{mol})$, tert-Butyl (E)-3(o-tolyloxy)-3-(4-(trifluoromethyl)phenyl)acrylate $(0.13 \mathrm{~g}, 0.36 \mathrm{mmol})$, and $\mathrm{CH}_{2} \mathrm{Cl}_{2}(0.80$ $\mathrm{mL}$ ). After $72 \mathrm{~h}$, the reaction was complete and the crude product was purified by column chromatography, using ethyl acetate/heptane as eluent to afford the title compound (0.13 g, 96\%) as a clear oil. ${ }^{1} \mathrm{H}$ NMR $(400 \mathrm{MHz}$, Chloroform- $d) \delta 7.60(\mathrm{~d}, J=8.1 \mathrm{~Hz}, 2 \mathrm{H}), 7.52$ $(\mathrm{d}, J=8.1 \mathrm{~Hz}, 2 \mathrm{H}), 7.12(\mathrm{dt}, J=7.2,1.2 \mathrm{~Hz}, 1 \mathrm{H}), 7.03-6.93(\mathrm{~m}, 1 \mathrm{H}), 6.81(\mathrm{td}, J=7.4$, $1.1 \mathrm{~Hz}, 1 \mathrm{H}), 6.60(\mathrm{~d}, J=8.2 \mathrm{~Hz}, 1 \mathrm{H}), 5.66(\mathrm{dd}, J=8.8,4.8 \mathrm{~Hz}, 0 \mathrm{H}), 2.96(\mathrm{dd}, J=15.5,8.8$ $\mathrm{Hz}, 1 \mathrm{H}), 2.72$ (dd, $J=15.4,4.8 \mathrm{~Hz}, 1 \mathrm{H}), 2.28$ (s, 3H), $1.40(\mathrm{~s}, 9 \mathrm{H}) .{ }^{13} \mathrm{C}$ NMR $(101 \mathrm{MHz}$, Chloroform- $d$ ) $\delta 169.3(\mathrm{C}), 155.6(\mathrm{C}), 145.0(\mathrm{C}), 130.9(\mathrm{CH}), 130.3$ (q, $J=32.5 \mathrm{~Hz}, \mathrm{C}$ ), $127.3(\mathrm{C}), 126.8(\mathrm{CH}), 126.5(\mathrm{CH}), 124.1\left(\mathrm{q}, J=449.7 \mathrm{~Hz}, \mathrm{CF}_{3}\right), 125.9(\mathrm{q}, J=3.8 \mathrm{~Hz}$, $\mathrm{CH}), 121.0(\mathrm{CH}), 112.8(\mathrm{CH}), 81.4(\mathrm{C}), 76.2(\mathrm{CH}), 44.9\left(\mathrm{CH}_{2}\right), 28.1\left(\mathrm{CH}_{3}\right), 16.5\left(\mathrm{CH}_{3}\right) .{ }^{19} \mathrm{~F}$ NMR (376 MHz, Chloroform- $d$ ) $\delta-63.50 .[\alpha]_{\mathrm{D}}{ }^{23}-25.7\left(c 1.00, \mathrm{CHCl}_{3}\right)$ HRMS (FTMS) $m / z:[\mathrm{M}+\mathrm{Na}]^{+}$calcd for $\mathrm{C}_{21} \mathrm{H}_{23} \mathrm{~F}_{3} \mathrm{O}_{3} \mathrm{Na}$ 403.1492; found 403.1477. HPLC analysis (ChiralPak IA, part number 80325; 90\% hexanes, $10 \%$ (9:1) hexanes:EtOH (abs), isocratic, $1.0 \mathrm{~mL} / \mathrm{min}, 230 \mathrm{~nm}$ ) indicated $95 \%$ ee: $\mathrm{t}_{\mathrm{R}}($ major $)=5.0 \mathrm{~min}, \mathrm{t}_{\mathrm{R}}($ minor $)=5.5 \mathrm{~min}$. 


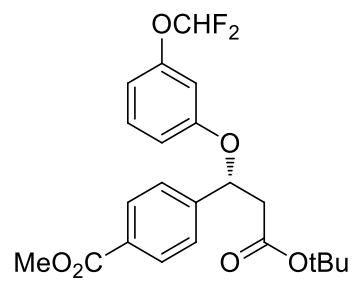

(R)-methyl 4-(3-(tert-butoxy)-1-(3-(difluoromethoxy)phenoxy)-3-oxopropyl)benzoate (23). Using Method $\mathrm{E}$, the following amounts of regents were used, bis(norbornadiene)rhodium(I) tetrafluoroborate $(0.089 \mathrm{mg}, 0.24 \mu \mathrm{mol})$ and J505-1 (0.15 $\mathrm{mg}, \quad 0.26 \mu \mathrm{mol}$ ), (E)-methyl 4-(3-(tert-butoxy)-1-(3-(difluoromethoxy)phenoxy)-3oxoprop-1-en-1-yl)benzoate $(0.20 \mathrm{~g}, 0.48 \mathrm{mmol})$, and $\mathrm{CH}_{2} \mathrm{Cl}_{2}(1.0 \mathrm{~mL})$. After $2 \mathrm{~h}$, the reaction was complete and the crude product was purified by column chromatography, using ethyl acetate/heptane as eluent to afford the title compound $(0.19 \mathrm{~g}, 95 \%)$ as a clear oil. ${ }^{1} \mathrm{H}$ NMR (400 MHz, Chloroform- $d$ ) $\delta 8.04-8.02(\mathrm{~m}, 1 \mathrm{H}), 8.01(\mathrm{~m}, 1 \mathrm{H}), 7.48-7.46$ (m, 1H), $7.46-7.44(\mathrm{~m}, 1 \mathrm{H}), 7.18-7.10(\mathrm{~m}, 1 \mathrm{H}), 6.68-6.61(\mathrm{~m}, 3 \mathrm{H}), 6.41(\mathrm{t}, J=74.0$ $\mathrm{Hz}, 1 \mathrm{H}), 5.61(\mathrm{dd}, J=9.0,4.7 \mathrm{~Hz}, 1 \mathrm{H}), 3.90(\mathrm{~s}, 3 \mathrm{H}), 2.93(\mathrm{dd}, J=15.6,9.0 \mathrm{~Hz}, 1 \mathrm{H}), 2.70$ (dd, $J=15.6,4.7 \mathrm{~Hz}, 1 \mathrm{H}), 1.41$ (s, 9H). ${ }^{13} \mathrm{C}$ NMR $(101 \mathrm{MHz}$, Chloroform- $d$ ) $\delta 169.2(\mathrm{C})$, $166.8(\mathrm{C}), 158.9(\mathrm{C}), 152.3(\mathrm{C}), 145.2(\mathrm{C}), 130.3(\mathrm{C}, J=9.6 \mathrm{~Hz}), 126.2(\mathrm{CH}), 116.0\left(\mathrm{CHF}_{2}\right.$, $J=260 \mathrm{~Hz}), 112.8(\mathrm{CH}), 112.0(\mathrm{CH}), 110.8(\mathrm{CH}), 107.9(\mathrm{CH}), 81.6(\mathrm{C}), 77.1(\mathrm{CH}) 52.3$ $\left(\mathrm{CH}_{3}\right), 44.7\left(\mathrm{CH}_{2}\right), 28.2\left(\mathrm{CH}_{3}\right) .{ }^{19} \mathrm{~F}$ NMR $(376 \mathrm{MHz}$, Chloroform- $d$ ) $\delta$-81.71. HRMS (FTMS) $m / z:[\mathrm{M}+\mathrm{Na}]^{+}$calcd for $\mathrm{C}_{22} \mathrm{H}_{24} \mathrm{~F}_{2} \mathrm{O}_{6} \mathrm{Na} 445.1433$; found 445.1422. $[\alpha]_{\mathrm{D}}{ }^{23}+1.7(c$ 1.18, $\mathrm{CHCl}_{3}$ ). HPLC analysis (ChiralPak IA, part number $80325 ; 85 \%$ Hexanes, $15 \%$ (9:1) Hexanes:EtOH (abs), isocratic, $1.0 \mathrm{~mL} / \mathrm{min}, 210 \mathrm{~nm}$ ) indicated $96 \%$ ee: $\mathrm{tR}$ (major) $=10.2$ min, $\mathrm{tR}($ minor $)=9.4 \mathrm{~min}$

\section{Scale-up of Compound (E)-8}

\subsection{Scale-up of Oxa-Michael Reaction}

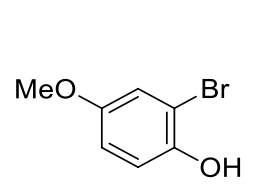

$9.1 \mathrm{~g}$
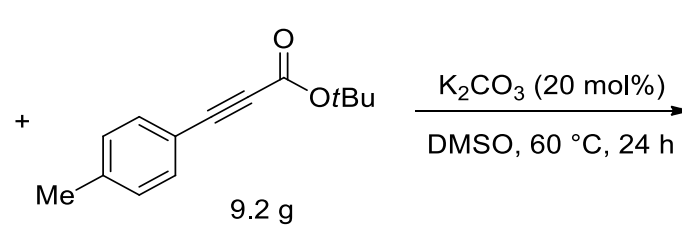

To a $250 \mathrm{~mL}$ round bottom flask was added 2-bromo-4-methoxyphenol $(9.07 \mathrm{~g}, 44.7$ $\mathrm{mmol})$, tert-butyl 3 -( $p$-tolyl)propiolate $(9.20 \mathrm{~g}, 42.5 \mathrm{mmol})$ and potassium carbonate $(1.18$ $\mathrm{g}, 8.50 \mathrm{mmol})$. The reaction mixture was diluted with DMSO $(42.5 \mathrm{~mL})$ and set to stir at $65{ }^{\circ} \mathrm{C}$ for $24 \mathrm{~h}$. Upon reaction completion as judged by the disappearance of the propiolate by HPLC, the reaction mixture was diluted with MTBE $(200 \mathrm{~mL})$ and $2.5 \mathrm{wt} \% \mathrm{aq} \mathrm{LiCl}$ $(200 \mathrm{~mL})$. The aqueous layer was extracted with MTBE $(2 \times 150 \mathrm{~mL})$ and the combined organics were washed with water $(200 \mathrm{~mL})$, brine $(200 \mathrm{~mL})$ and dried over $\mathrm{MgSO}_{4}$. The resulting organic layer was filtered through a slug of silica gel $(10 \mathrm{~cm})$ to remove the purple/brown color and give a light-yellow solution. Concentration in vacuo gave the product as a light brown solid (17.6 g, 99\%, 28:72 E/Z ratio). 


\subsection{Scale-up of Photoisomerization}<smiles>CCCOC(=O)/C=C(\Oc1ccc(OC)cc1Br)c1ccc(C)cc1</smiles>

$17.5 \mathrm{~g}$

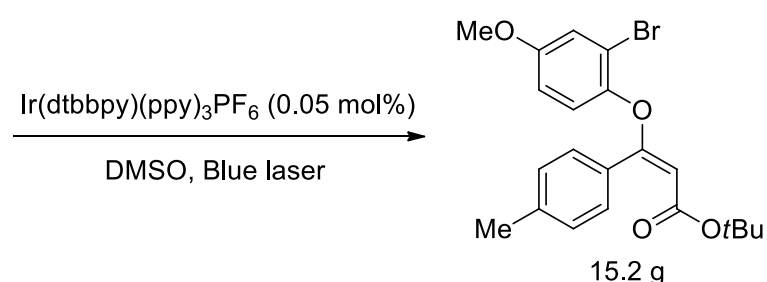

$15.2 \mathrm{~g}$

To a $500 \mathrm{~mL}$ jacketed containing tert-butyl 3-(2-bromo-4-methoxyphenoxy)-3-( $p$ tolyl)acrylate $(17.6 \mathrm{~g}, 41.9 \mathrm{mmol})$ was added DMSO $(280 \mathrm{~mL})$ and the mixture was allowed to stir until a homogeneous solution was obtained. $\operatorname{Ir}(\mathrm{dtbbpy})(\mathrm{ppy})_{3} \mathrm{PF}_{6}(0.019 \mathrm{~g}$, $0.021 \mathrm{mmol}$ ) was added and the photoisomerization step was conducted with a $25 \mathrm{~W}$ laser as described by Harper et al. ${ }^{1}$ at $60{ }^{\circ} \mathrm{C}$ for $7 \mathrm{~h}$ (Figure S6). At 96:4 E/Z ratio, the product was crystallized from solution by the dropwise addition of water $(30 \mathrm{~mL})$ while stirring. The resulting slurry was let stir at RT for $16 \mathrm{~h}$ and then filtered. The filtrate was washed with 1:1 DMSO/water $(30 \mathrm{~mL})$ and water $(4 \times 100 \mathrm{~mL})$ and dried under vacuum to give the pure E-isomer as a white solid $(15.2 \mathrm{~g}, 87 \%)$. Characterization data matched compound (E)-8 above.

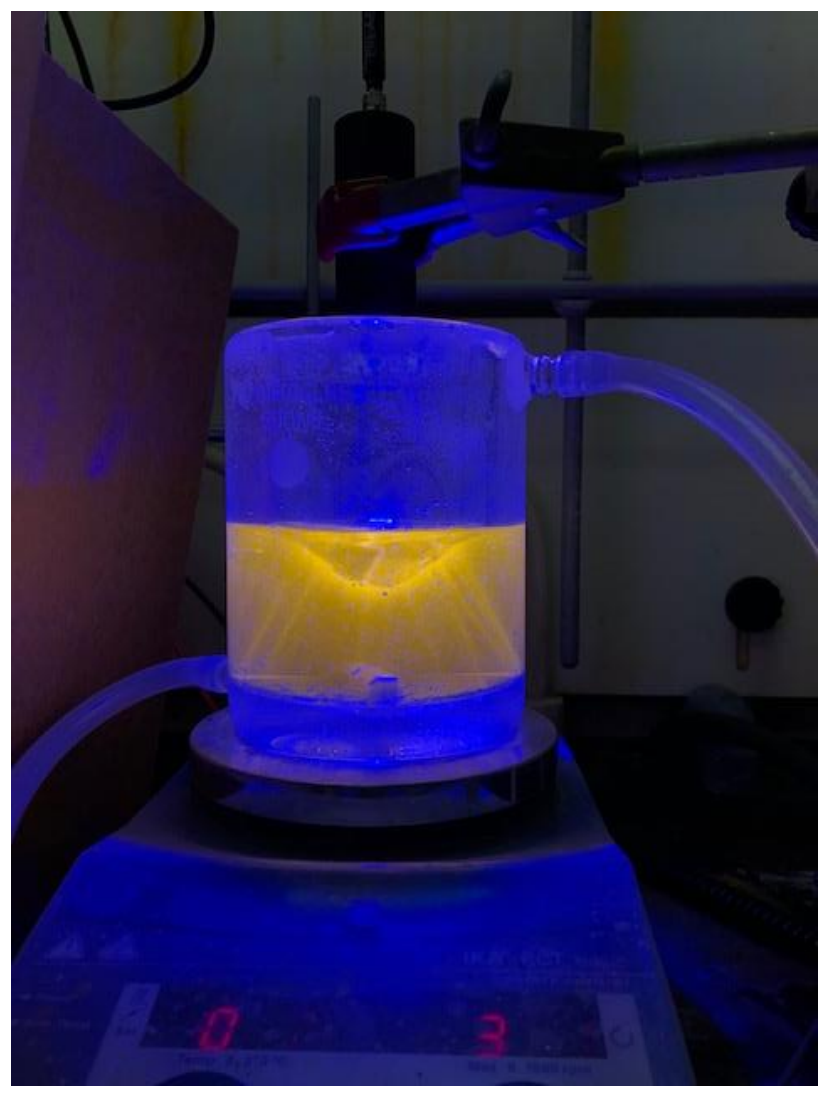

Figure S6. Scale up of isomerization under $25 \mathrm{~W}$ laser irradiation. 


\subsection{Scale-up of Asymmetric Hydrogenation}
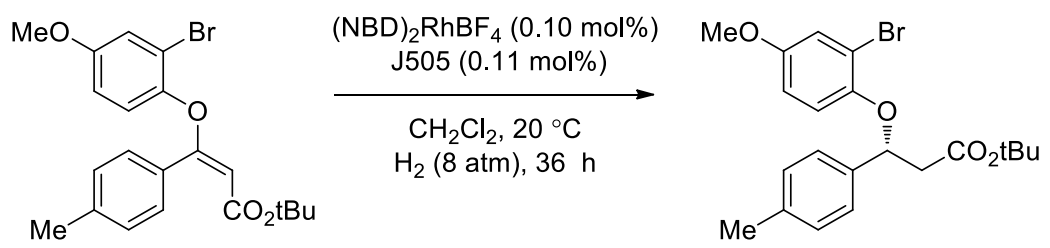

A one-dram vial was charged with bis(norbornadiene)rhodium(I) tetrafluoroborate (5.60 $\mathrm{mg}, 15.0 \mu \mathrm{mol})$ and J505-1 (9.40 mg, $16.4 \mu \mathrm{mol})$. Inside the glove box, the catalyst and (E)-tert-butyl 3-(2-bromo-4-methoxyphenoxy)-3-(p-tolyl)acrylate ( $6.28 \mathrm{~g}, 15.0 \mathrm{mmol}$ ) was transferred using $\mathrm{CH}_{2} \mathrm{Cl}_{2}(31 \mathrm{ml})$ to a $50 \mathrm{~mL}$ stirred Parr reactor. The reactor was sealed, placed in a reactor stand, and pressurized to $8 \mathrm{~atm}$ with hydrogen. The mixture was stirred at RT and was judged complete after $36 \mathrm{~h}$. Upon reaction completion, the mixture was concentrated to give the product as an orange oil $(6.0 \mathrm{~g}, 95 \%$ yield, $97 \%$ ee). Characterization data matched compound $\mathbf{2 1}$ above.

\section{References}

1. Harper, K. C.; Moschetta, E. G.; Bordawekar, S. V.; Wittenberger, S. J., A Laser Driven Flow Chemistry Platform for Scaling Photochemical Reactions with Visible Light. ACS Cent. Sci. 2019, 5, 109-115.

2. $\quad$ For triplet state energies of photocatalysts see: a) Zhou, Q.-Q.; Zou, Y.-Q.; Lu, L.Q.; Xiao, W.-J. Visible-Light-Induced Organic Photochemical Reactions through EnergyTransfer Pathways. Angew. Chem., Int. Ed. 2019, 58, 1586-1604. b) Singh, A.; Teegardin, K.; Kelly, M.; Prasad, K. S.; Krishnan, S.; Weaver, J. D. Facile Synthesis and Complete Characterization of Homoleptic and Heteroleptic Cyclometalated Iridium(III) Complexes for Photocatalysis. J. Organomet. Chem. 2015, 776, 51-59. c) Metternich, J. B.; Gilmour, R. A Bio-Inspired, Catalytic E $\rightarrow$ Z Isomerization of Activated Olefins. J. Am. Chem. Soc. 2015, 137, 11254-11257.

3. Janková, Š.; Dračínský, M.; Císařová, I.; Kotora, M., Synthesis and Rearrangement of Dewar Benzenes Into Biaryls: Experimental Evidence for Conrotatory Ring Opening. Eur. J. Org. Chem. 2008, 2008, 47-51.

4. Schilz, M.; Plenio, H., A Guide to Sonogashira Cross-Coupling Reactions: The Influence of Substituents in Aryl Bromides, Acetylenes, and Phosphines. J. Org. Chem. 2012, 77, 2798-2807.

5. $\quad$ Singh, B. K.; Kaval, N.; Tomar, S.; Eycken, E. V.; Parmar, V. S., Transition MetalCatalyzed Carbon-Carbon Bond Formation Suzuki, Heck, and Sonogashira Reactions Using Microwave and Microtechnology. Org. Proc. Res. Dev. 2008, 12, 468-474.

\section{Crystallographic Data for 15 Single Crystal X-ray Structure}

\section{Experimental procedure for sample preparation and single crystal X-ray diffraction} studies 
Suitable single crystals of $\mathbf{1 5}$ were prepared by dissolution in methylcyclohexane at 35 ${ }^{\circ} \mathrm{C}$, followed by cooling to ambient temperature and slow evaporation. An elongated plate-shaped single crystal of $\mathbf{1 5}$ was cut to the appropriate length and mounted on a loop. Intensity data were collected on a Bruker APEX diffractometer equipped with a PhotonII detector. Data were collected at $100 \mathrm{~K}$ with graphite-monochromated $\mathrm{CuK}_{\alpha}$ radiation $(\lambda=$ $1.54178 \AA$ A) using omega-phi scans. Data processing was carried out using the APEX3 software package. ${ }^{1}$ Corrections for Lorentz-polarization effects and absorption were applied. The structure was solved using the intrinsic phasing method to produce the nonhydrogen atoms initial structure. All presented hydrogen atoms were located in Fourierdifference electron density maps. All non-hydrogen atoms were refined anisotropically. Hydrogen atoms associated with carbon atoms were refined in geometrically constrained riding positions. Refinement was carried out with the use of SHELX-2016/6. ${ }^{2}$ Absolute stereochemistry was determined by anomalous dispersion. ${ }^{3}$

Table S7. Structure parameters for 15

\begin{tabular}{cccccc}
\hline \multicolumn{2}{c}{ Space Group } & \multicolumn{2}{c}{$\mathrm{P} 2{ }_{1} / \mathrm{n}$} & \multicolumn{2}{c}{ Flack $=-0.005$} \\
\hline $\mathrm{a}$ & $6.22240(10) \AA$ & $\alpha$ & $90^{\circ}$ & $\mathrm{V}$ & $1876.45(7)$ \\
$\mathrm{b}$ & $17.1348(4) \AA$ & $\beta$ & $90^{\circ}$ & $\mathrm{Z} / \mathrm{Z}$ & $1 / 4$ \\
$\mathrm{c}$ & $17.5995(4) \AA$ & $\gamma$ & $90^{\circ}$ & $\mathrm{R}(\%)$ & 2.97 \\
\hline
\end{tabular}

${ }^{1}$ Bruker AXS Inc., APEX3,version 2018.7-2, Madision Wisconsin, 2018.

${ }^{2}$ Sheldrick, G.M., Acta Cryst. (2015), C71, 3-8.

${ }^{3}$ Parsons, Flack and Wagner, Acta Cryst. (2013), B69, 249-259. 


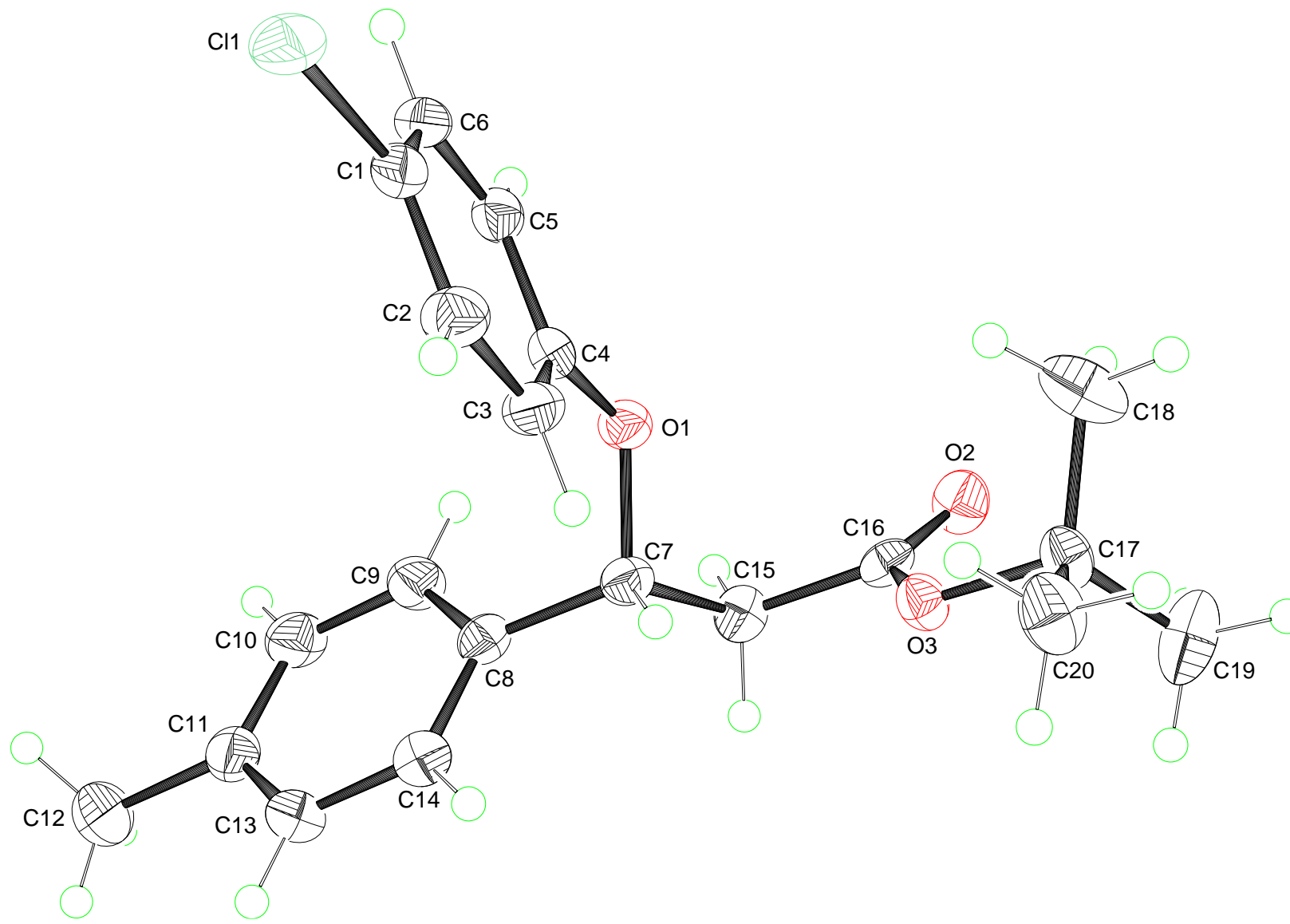

Figure S7. Thermal ellipsoid plot (ORTEP) of 15. Ellipsoids drawn at 50\% probability.

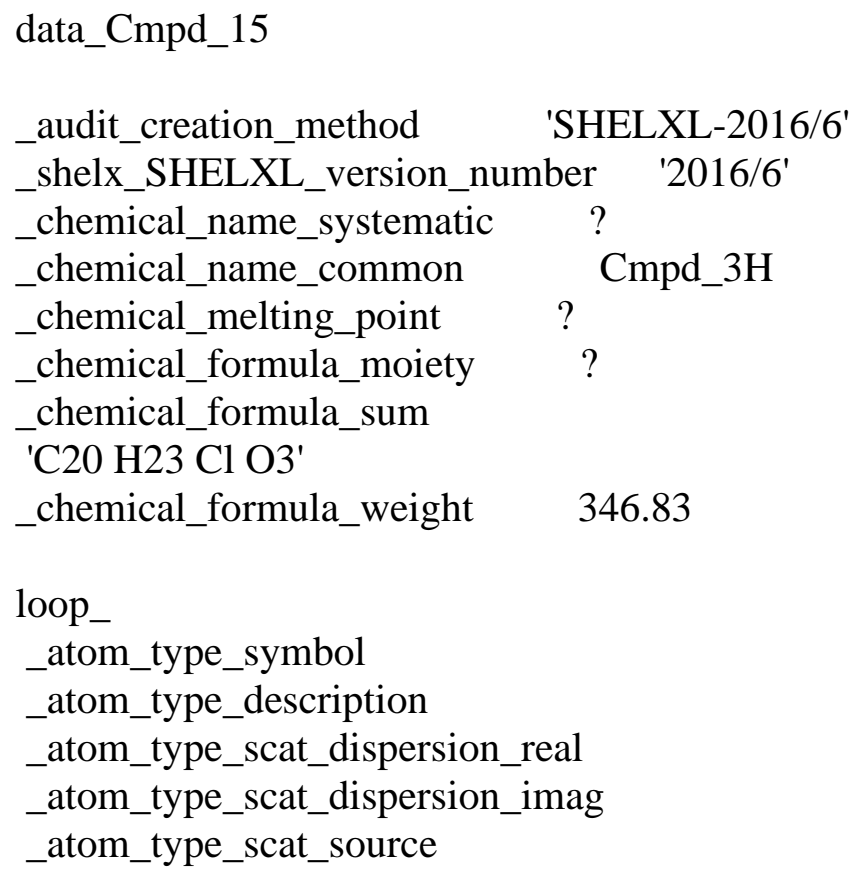


'C' 'C' $0.0181 \quad 0.0091$

'International Tables Vol C Tables 4.2.6.8 and 6.1.1.4'

'H' 'H' $0.0000 \quad 0.0000$

'International Tables Vol C Tables 4.2.6.8 and 6.1.1.4'

'Cl' 'Cl' 0.36390 .7018

'International Tables Vol C Tables 4.2.6.8 and 6.1.1.4'

'O' 'O' $0.0492 \quad 0.0322$

'International Tables Vol C Tables 4.2.6.8 and 6.1.1.4'

_space_group_crystal_system

_space_group_IT_number

orthorhombic

_space_group_name_H-M_alt

_space_group_name_Hall

19

'P 2121 21'

'P 2ac 2ab'

_shelx_space_group_comment

;

The symmetry employed for this shelxl refinement is uniquely defined by the following loop, which should always be used as a source of symmetry information in preference to the above space-group names.

They are only intended as comments.

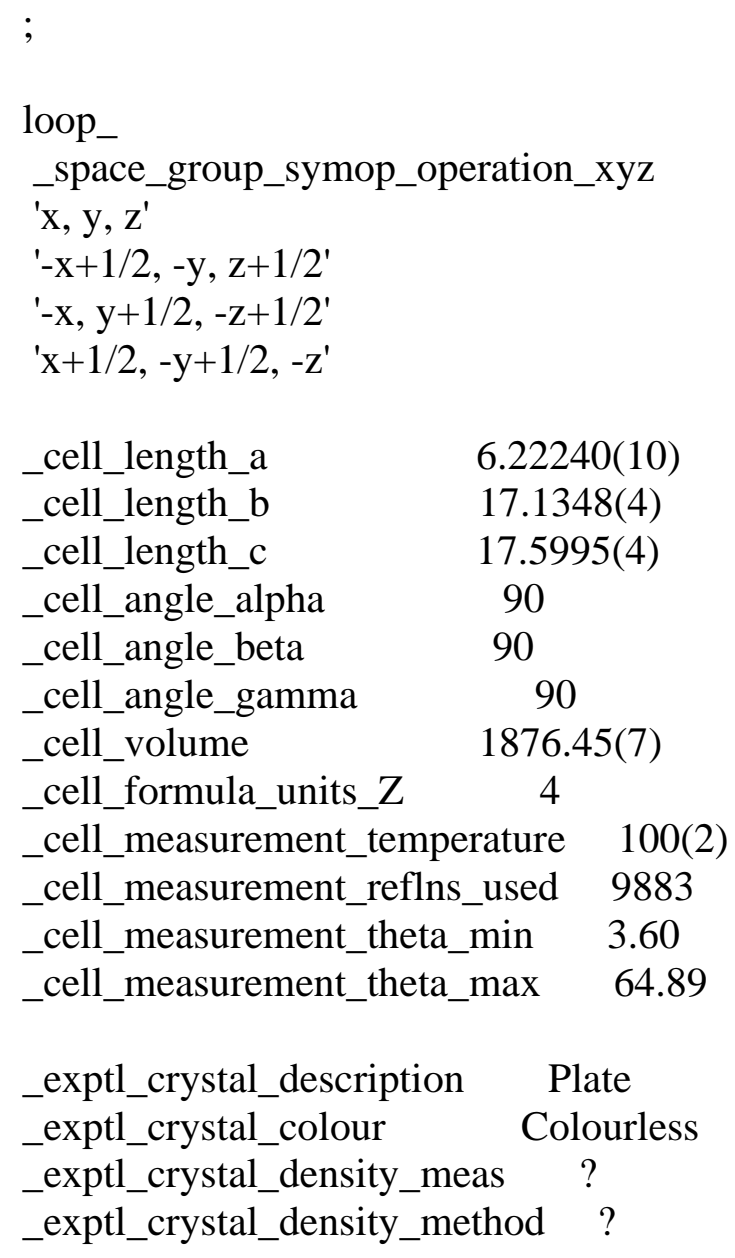




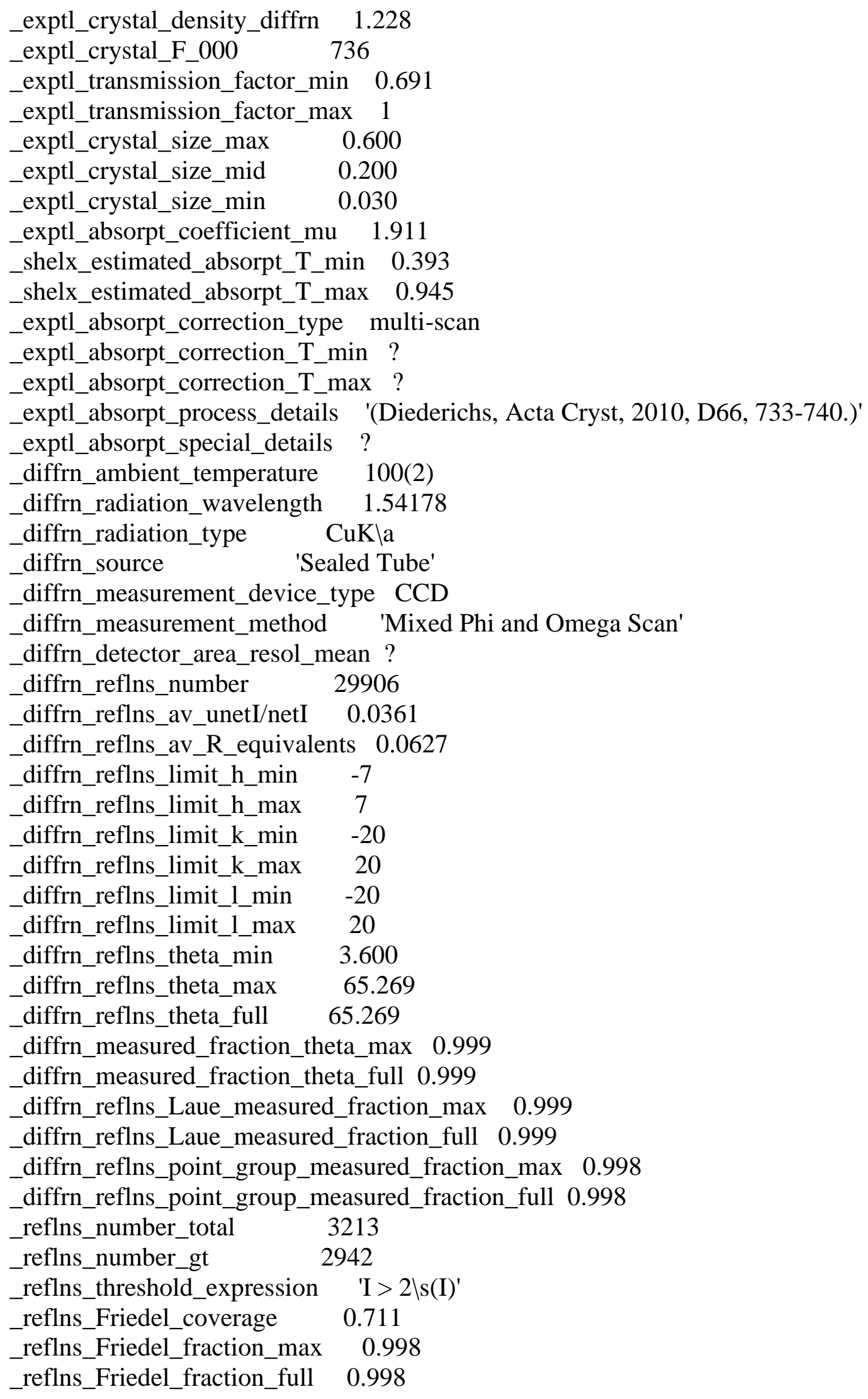


_reflns_special_details

;

Reflections were merged by SHELXL according to the crystal

class for the calculation of statistics and refinement.

_reflns_Friedel_fraction is defined as the number of unique

Friedel pairs measured divided by the number that would be

possible theoretically, ignoring centric projections and

systematic absences.

_computing_data_collection ?

_computing_cell_refinement ?

_computing_data_reduction ?

_computing_structure_solution＇SHELXT 2014/5 (Sheldrick, 2014)'

_computing_structure_refinement 'SHELXL-2016/6 (Sheldrick, 2016)'

_computing_molecular_graphics ?

_computing_publication_material ?

_refine_special_details?

_refine_ls_structure_factor_coef Fsqd

_refine_ls_matrix_type full

_refine_ls_weighting_scheme calc

_refine_ls_weighting_details

$' \mathrm{w}=1 /\left[\mathrm{s}^{\wedge} 2^{\wedge}\left(\mathrm{Fo}^{\wedge} 2^{\wedge}\right)+(0.0293 \mathrm{P})^{\wedge} 2^{\wedge}+0.3629 \mathrm{P}\right]$ where $\mathrm{P}=\left(\mathrm{Fo}^{\wedge} 2^{\wedge}+2 \mathrm{Fc}^{\wedge} 2^{\wedge}\right) / 3^{\prime}$

_atom_sites_solution_primary ?

_atom_sites_solution_secondary ?

_atom_sites_solution_hydrogens geom

_refine_ls_hydrogen_treatment constr

_refine_ls_extinction_method none

_refine_1s_extinction_coef .

_refine_ls_abs_structure_details

;

Flack x determined using 1180 quotients [(I+)-(I-)]/[(I+)+(I-)]

(Parsons, Flack and Wagner, Acta Cryst. B69 (2013) 249-259).

;

_refine_1s_abs_structure_Flack $-0.005(6)$

_chemical_absolute_configuration ad

_refine_ls_number_reflns 3213

_refine_ls_number_parameters 221

_refine_ls_number_restraints 0

_refine_ls_R_factor_all 0.0344

_refine_ls_R_factor_gt 0.0295

_refine_ls_wR_factor_ref 0.0706

_refine_ls_wR_factor_gt 0.0675

_refine_ls_goodness_of_fit_ref 1.053 
_refine_ls_restrained_S_all 1.053

_refine_ls_shift/su_max $\quad 0.000$

_refine_ls_shift/su_mean $\quad 0.000$

loop_

_atom_site_label

_atom_site_type_symbol

_atom_site_fract_x

_atom_site_fract_y

_atom_site_fract_z

_atom_site_U_iso_or_equiv

_atom_site_adp_type

_atom_site_occupancy

_atom_site_site_symmetry_order

_atom_site_calc_flag

_atom_site_refinement_flags_posn

_atom_site_refinement_flags_adp

_atom_site_refinement_flags_occupancy

_atom_site_disorder_assembly

_atom_site_disorder_group

Cl1 Cl 0.07859(11) 0.44821(4) 0.51170(3) 0.03930(18) Uani $11 \mathrm{~d} . . .$.

O1 O 0.7106(2) 0.45568(9) 0.76008(8) 0.0255(3) Uani $11 \mathrm{~d} \ldots .$.

O2 O 1.0974(3) 0.57842(10) 0.85140(10) 0.0338(4) Uani $11 \mathrm{~d} \ldots \ldots$

O3 O 0.7466(3) 0.60651(9) 0.86913(9) 0.0260(3) Uani $11 \mathrm{~d} \ldots .$.

C1 C 0.2622(4) 0.45222(15) 0.58678(12) 0.0293(5) Uani $11 \mathrm{~d} . . .$.

C2 C 0.1999(4) 0.48146(15) 0.65643(14) 0.0324(5) Uani $11 \mathrm{~d}$......

H2 H 0.0582260 .5008210 .6634070 .039 Uiso 11 calc R U . . .

C3 C 0.3456(4) 0.48244(15) 0.71640(14) 0.0291(5) Uani $11 \mathrm{~d}$. . . . .

H3 H 0.3037260 .5024100 .7645310 .035 Uiso 11 calc R U . . .

C4 C 0.5522(4) 0.45415(13) 0.70563(12) 0.0240(5) Uani $11 \mathrm{~d} \ldots .$.

C5 C 0.6119(4) 0.42502(12) 0.63539(13) 0.0275(5) Uani $11 \mathrm{~d} . . .$.

H5 H 0.7532160 .4053650 .6282180 .033 Uiso 11 calc R U . . .

C6 C 0.4679(4) 0.42424(14) 0.57553(13) 0.0300(5) Uani $11 \mathrm{~d}$. . . .

H6 H 0.5100210 .4046970 .5272550 .036 Uiso 11 calc R U . . .

C7 C 0.6474(4) 0.45143(14) 0.83932(12) 0.0246(5) Uani $11 \mathrm{~d} . . .$.

H7 H 0.5282790 .4892220 .8493310 .029 Uiso 11 calc R U . . .

C8 C 0.5760(4) 0.36998(13) 0.86016(12) 0.0250(5) Uani $11 \mathrm{~d}$. . . . .

C9 C 0.6950(4) 0.30469(14) 0.83957(14) 0.0324(5) Uani $11 \mathrm{~d}$. . . . .

H9 H 0.8207210 .3108010 .8095420 .039 Uiso 11 calc R U . . .

C10 C 0.6321(4) 0.23091(15) 0.86233(15) 0.0355(6) Uani 11 d . . . .

H10 H 0.7162570 .1870850 .8478920 .043 Uiso 11 calc R U . . .

C11 C 0.4491(4) 0.21954(14) 0.90574(14) 0.0322(5) Uani $11 \mathrm{~d} . . .$.

C12 C 0.3811(5) 0.13877(17) 0.93031(17) 0.0458(7) Uani $11 \mathrm{~d} . . .$.

H12A H 0.2577500 .1427100 .9647280 .069 Uiso 11 calc R U . . .

H12B H 0.5006140 .1131290 .9566100 .069 Uiso 11 calc R U . . .

H12C H 0.3407650 .1080760 .8855450 .069 Uiso 11 calc R U . . . 
C13 C 0.3303(4) 0.28465(15) 0.92649(14) 0.0328(5) Uani $11 \mathrm{~d} \ldots .$. H13 H 0.2048510 .2783590 .9566470 .039 Uiso 11 calc R U ... C14 C 0.3924(4) 0.35893(14) 0.90372(13) 0.0280(5) Uani 11 d . . . . . H14 H 0.3081090 .4027340 .9181310 .034 Uiso 11 calc R U . . . C15 C 0.8473(4) 0.47636(13) 0.88232(14) 0.0278(5) Uani 11 d ..... H15A H 0.8198240 .4716570 .9375340 .033 Uiso 11 calc R U . . . H15B H 0.9665710 .4404850 .8693750 .033 Uiso 11 calc R U . . . C16 C 0.9156(4) 0.55909(14) 0.86484(11) 0.0242(4) Uani 11 d . . . . . C17 C 0.7607(4) 0.69070(13) 0.85057(14) 0.0297(5) Uani $11 \mathrm{~d}$. . . . . C18 C 0.8507(5) 0.7014(2) 0.77140(18) 0.0508(8) Uani $11 \mathrm{~d}$. . . . . H18A H 1.0047460 .6893820 .7716140 .076 Uiso 11 calc R U . . . H18B H 0.8291430 .7554720 .7550750 .076 Uiso 11 calc R U . . . H18C H 0.7766760 .6661150 .7362130 .076 Uiso 11 calc R U . . . C19 C 0.8920(5) 0.73099(17) 0.9106(2) 0.0506(8) Uani $11 \mathrm{~d}$. . . . . H19A H 0.8292760 .7207750 .9606530 .076 Uiso 11 calc R U . . . H19B H 0.8927410 .7873160 .9009200 .076 Uiso 11 calc R U . . . H19C H 1.0396710 .7111340 .9092040 .076 Uiso 11 calc R U . . . C20 C 0.5267(4) 0.71537(15) 0.85377(18) 0.0394(6) Uani $11 \mathrm{~d}$..... H20A H 0.4443460 .6860200 .8158520 .059 Uiso 11 calc R U . . . H20B H 0.5155090 .7713340 .8429850 .059 Uiso 11 calc R U . . . H20C H 0.4689530 .7046850 .9045230 .059 Uiso 11 calc R U . . .

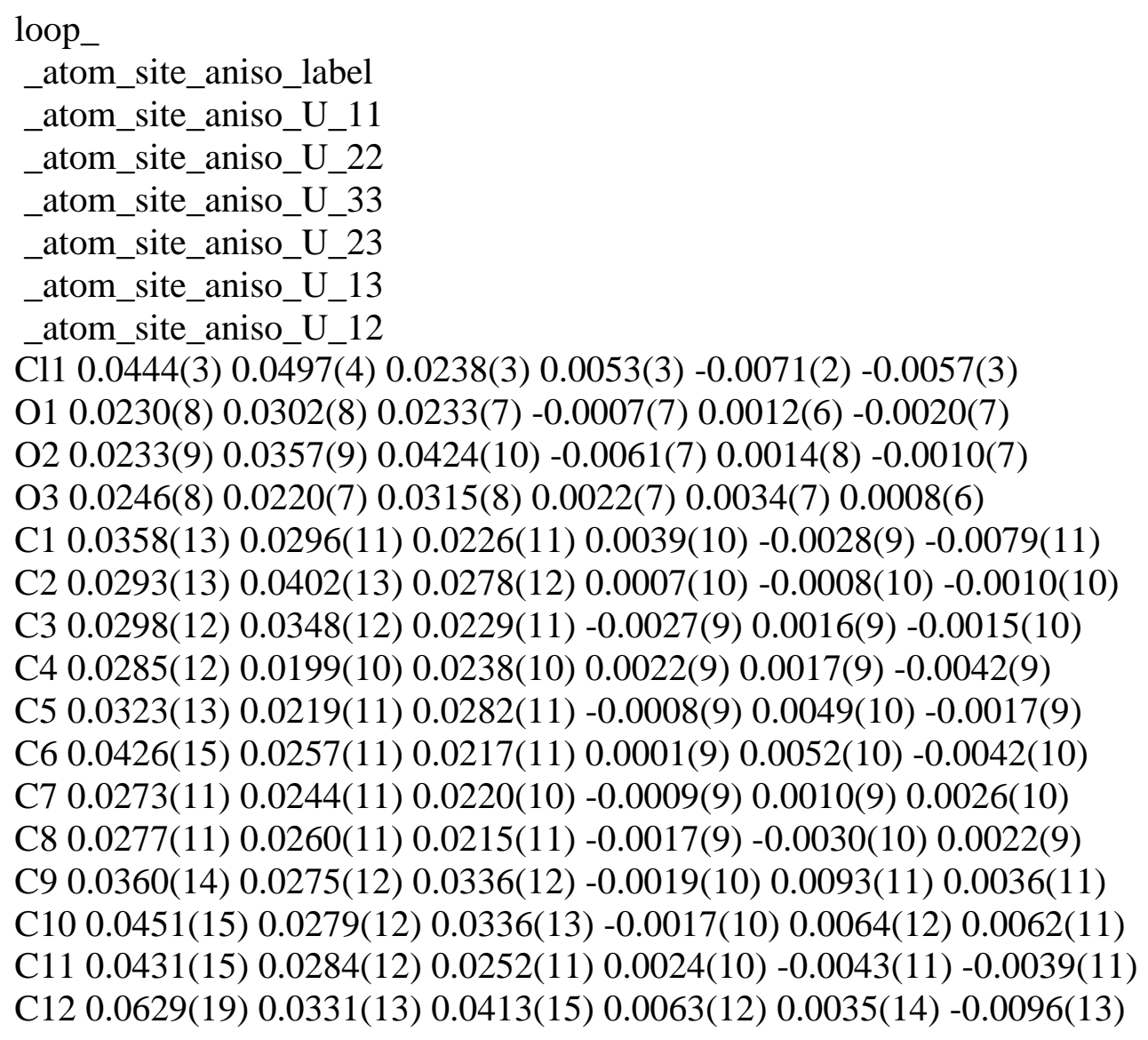


C13 0.0348(13) 0.0358(13) 0.0279(12) 0.0022(10) 0.0048(10) -0.0043(10)

C14 0.0264(12) 0.0303(12) 0.0273(11) -0.0010(9) -0.0012(10) 0.0024(10)

C15 0.0281(12) 0.0257(12) 0.0298(12) $-0.0005(9)-0.0034(10) 0.0032(9)$

C16 0.0229(11) 0.0302(11) 0.0195(10) $-0.0055(9)-0.0032(9)$ 0.0024(10)

C17 0.0325(12) 0.0213(11) 0.0354(13) 0.0046(9) 0.0004(10) -0.0004(10)

C18 0.0478(17) 0.0581(18) 0.0464(16) 0.0238(14) 0.0085(14) 0.0033(14)

C19 0.0512(18) 0.0322(14) 0.068(2) -0.0148(13) -0.0184(16) 0.0044(13)

C20 0.0342(14) 0.0290(13) 0.0552(17) 0.0055(12) 0.0006(12) 0.0052(11)

_geom_special_details

;

All esds (except the esd in the dihedral angle between two 1.s. planes) are estimated using the full covariance matrix. The cell esds are taken into account individually in the estimation of esds in distances, angles and torsion angles; correlations between esds in cell parameters are only used when they are defined by crystal symmetry. An approximate (isotropic) treatment of cell esds is used for estimating esds involving l.s. planes. ;

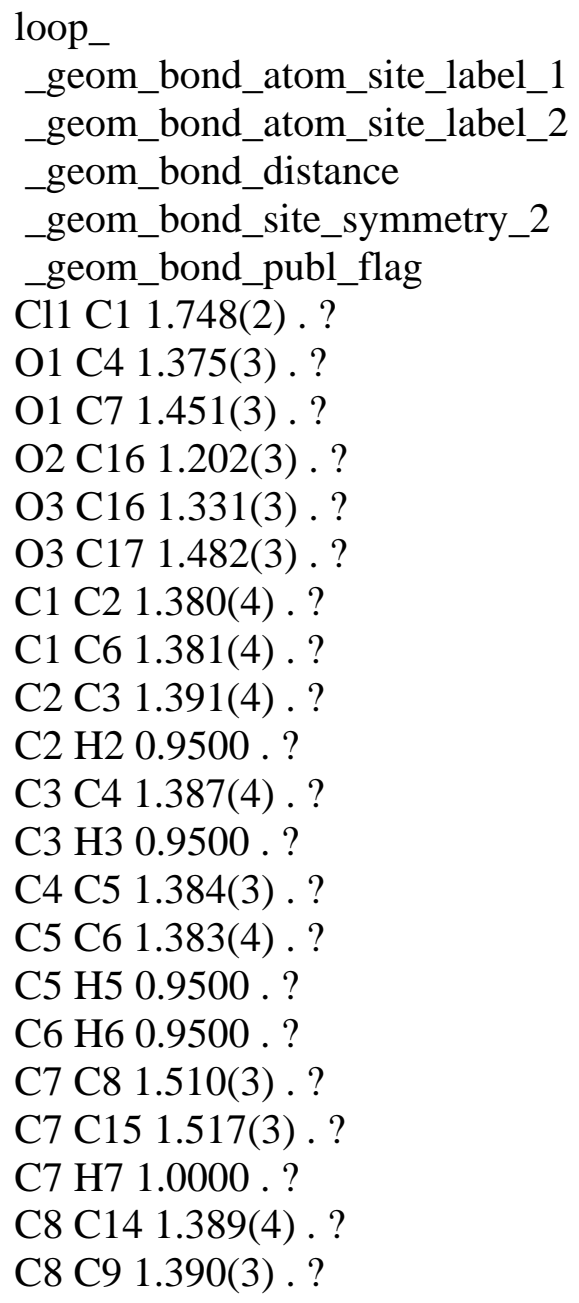


C9 C10 1.383(4) . ?

C9 H9 0.9500 . ?

C10 C11 1.385(4) . ?

C10 H10 0.9500 . ?

C11 C13 1.387(4) . ?

C11 C12 1.510(4) . ?

C12 H12A 0.9800 . ?

C12 H12B 0.9800 . ?

C12 H12C 0.9800 . ?

C13 C14 1.389(4) . ?

C13 H13 0.9500.?

C14 H14 0.9500 . ?

C15 C16 1.512(3) . ?

C15 H15A 0.9900 . ?

C15 H15B 0.9900.?

C17 C19 1.503(4) . ?

C17 C18 1.513(4) . ?

C17 C20 1.517(4) . ?

C18 H18A 0.9800 . ?

C18 H18B 0.9800.?

C18 H18C 0.9800.?

C19 H19A 0.9800 . ?

C19 H19B 0.9800 . ?

C19 H19C 0.9800 . ?

C20 H20A 0.9800 . ?

C20 H20B 0.9800.?

$\mathrm{C} 20 \mathrm{H} 20 \mathrm{C} 0.9800$. ?

loop_

_geom_angle_atom_site_label_1

_geom_angle_atom_site_label_2

_geom_angle_atom_site_label_3

_geom_angle

_geom_angle_site_symmetry_1

_geom_angle_site_symmetry_3

_geom_angle_publ_flag

C4 O1 C7 118.36(16) ..?

C16 O3 C17 122.34(18) .. ?

C2 C1 C6 120.9(2) .. ?

C2 C1 Cl1 120.1(2) . .?

C6 C1 Cl1 118.92(18) . . ?

C1 C2 C3 119.7(2) .. ?

C1 C2 H2 120.2 . ?

C3 C2 H2 120.2 .. ?

C4 C3 C2 119.7(2) . . ?

C4 C3 H3 120.1 . . ? 
C2 C3 H3 120.1 .. ?

O1 C4 C5 115.9(2) . . ?

O1 C4 C3 124.2(2)..?

C5 C4 C3 119.8(2) .. ?

C6 C5 C4 120.7(2) .. ?

C6 C5 H5 119.7 . . ?

C4 C5 H5 119.7 . . ?

C1 C6 C5 119.2(2) .. ?

C1 C6 H6 120.4 . . ?

C5 C6 H6 120.4 .. ?

O1 C7 C8 111.08(18) .. ?

O1 C7 C15 104.07(18) .. ?

C8 C7 C15 112.35(19) .. ?

O1 C7 H7 109.7 . . ?

C8 C7 H7 109.7 .. ?

C15 C7 H7 109.7 ..?

C14 C8 C9 118.2(2) . . ?

C14 C8 C7 120.1(2) . . ?

C9 C8 C7 121.6(2) .. ?

C10 C9 C8 120.7(2) . . ?

C10 C9 H9 119.7 .. ?

C8 C9 H9 119.7 . . ?

C9 C10 C11 121.4(2) . . ?

C9 C10 H10 119.3 .. ?

C11 C10 H10 119.3 . . ?

C10 C11 C13 118.0(2) . . ?

C10 C11 C12 121.1(2) . . ?

C13 C11 C12 120.8(2) . . ?

C11 C12 H12A 109.5 . . ?

C11 C12 H12B 109.5 . .?

H12A C12 H12B 109.5 . .?

C11 C12 H12C 109.5 . . ?

H12A C12 H12C 109.5 . . ?

H12B C12 H12C 109.5 . . ?

C11 C13 C14 120.8(2) .. ?

C11 C13 H13 119.6 . . ?

C14 C13 H13 119.6 . ?

C8 C14 C13 120.9(2) ..?

C8 C14 H14 119.6 .. ?

C13 C14 H14 119.6 . . ?

C16 C15 C7 113.14(19) .. ?

C16 C15 H15A 109.0 . . ?

C7 C15 H15A 109.0 . .?

C16 C15 H15B 109.0 . . ?

C7 C15 H15B 109.0 . . ?

H15A C15 H15B 107.8 . . ? 
O2 C16 O3 125.9(2) . ?

O2 C16 C15 124.3(2) . .?

O3 C16 C15 109.80(18) . . ?

O3 C17 C19 108.9(2) . . ?

O3 C17 C18 110.0(2) . . ?

C19 C17 C18 113.0(3) .. ?

O3 C17 C20 101.91(19) .. ?

C19 C17 C20 111.6(2) . . ?

C18 C17 C20 110.9(2) . . ?

C17 C18 H18A 109.5 . . ?

C17 C18 H18B 109.5 .. ?

H18A C18 H18B 109.5 . . ?

C17 C18 H18C 109.5 ..?

H18A C18 H18C 109.5 . . ?

H18B C18 H18C 109.5 ..?

C17 C19 H19A 109.5 . . ?

C17 C19 H19B 109.5 .. ?

H19A C19 H19B 109.5 . . ?

C17 C19 H19C 109.5 . . ?

H19A C19 H19C 109.5 . . ?

H19B C19 H19C 109.5 . . ?

C17 C20 H20A 109.5 . . ?

C17 C20 H20B 109.5 .. ?

H20A C20 H20B 109.5 . .?

C17 C20 H20C 109.5 .. ?

H20A C20 H20C 109.5 . . ?

H20B C20 H20C 109.5 . . ?

loop_

_geom_torsion_atom_site_label_1

_geom_torsion_atom_site_label_2

_geom_torsion_atom_site_label_3

_geom_torsion_atom_site_label_4

geom_torsion

_geom_torsion_site_symmetry_1

_geom_torsion_site_symmetry_2

_geom_torsion_site_symmetry_3

_geom_torsion_site_symmetry_4

_geom_torsion_publ_flag

C6 C1 C2 C3 -0.4(4) ....?

C11 C1 C2 C3 178.26(19) .... ?

C1 C2 C3 C4 0.1(4)....?

C7 O1 C4 C5 -153.1(2).... ?

C7 O1 C4 C3 29.7(3) .... ?

C2 C3 C4 O1 177.0(2) ... . ?

$\mathrm{C} 2 \mathrm{C} 3 \mathrm{C} 4 \mathrm{C} 5-0.1(3) \ldots$ ? 
O1 C4 C5 C6 -176.96(19) .... ?

C3 C4 C5 C6 0.4(3) .... . ?

C2 C1 C6 C5 0.6(4) ....?

C11 C1 C6 C5 -178.00(17) .... ?

C4 C5 C6 C1 -0.7(3) ....?

$\mathrm{C} 4 \mathrm{O} 1 \mathrm{C} 7 \mathrm{C} 873.7(2) \ldots$ ?

C4 O1 C7 C15 -165.15(18) .... ?

O1 C7 C8 C14 -135.9(2) .... ?

C15 C7 C8 C14 108.0(2) .... ?

O1 C7 C8 C9 46.9(3) ....?

C15 C7 C8 C9 -69.2(3) ... . ?

C14 C8 C9 C10 -0.4(4) .... ?

C7 C8 C9 C10 176.9(2) . . . . ?

C8 C9 C10 C11 0.4(4) .... ?

C9 C10 C11 C13 -0.5(4) ....?

C9 C10 C11 C12 179.9(3) .... ?

C10 C11 C13 C14 0.6(4) .... ?

C12 C11 C13 C14 -179.8(2) ... . ?

C9 C8 C14 C13 0.4(3) ....?

C7 C8 C14 C13 -176.8(2) .... ?

C11 C13 C14 C8 -0.6(4) .... ? ?

O1 C7 C15 C16 62.3(2) ....?

C8 C7 C15 C16 -177.43(19) .... ?

$\mathrm{C} 17 \mathrm{O} 3 \mathrm{C} 16 \mathrm{O} 25.6(3) \ldots$ ?

C17 O3 C16 C15 -176.42(19) .... ?

C7 C15 C16 O2 -134.2(2) ....?

C7 C15 C16 O3 47.8(2) ....?

C16 O3 C17 C19-69.3(3) ....?

C16 O3 C17 C18 55.1(3) ....?

C16 O3 C17 C20 172.7(2) ....?

_refine_diff_density_max 0.168

_refine_diff_density_min -0.200

_refine_diff_density_rms 0.039

\section{NMR and HPLC Data for New Compounds}


1H NMR (400 MHz, Chloroform-d)

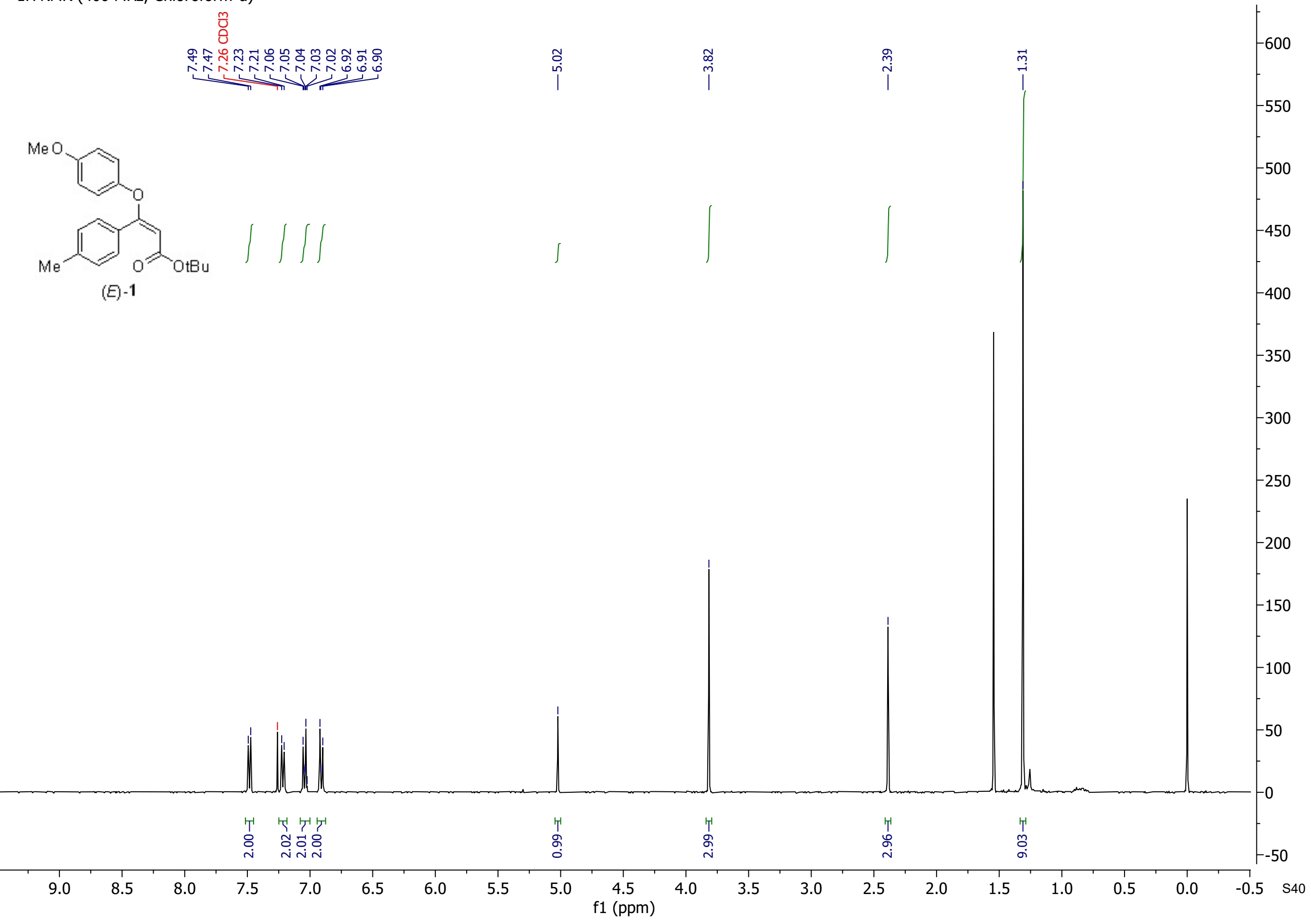


13C NMR (101 MHz, Chloroform-d)

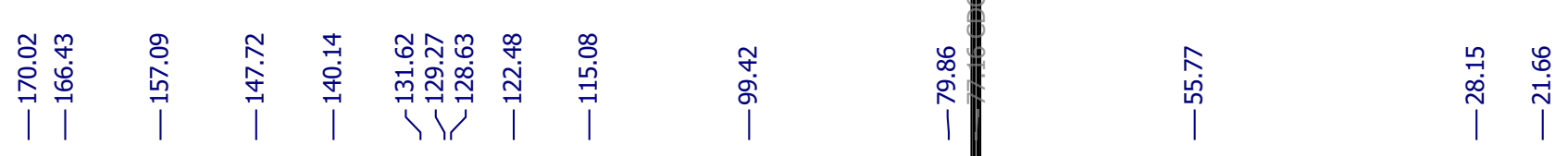

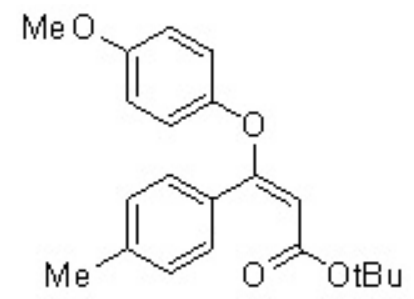

$(E)-1$ 


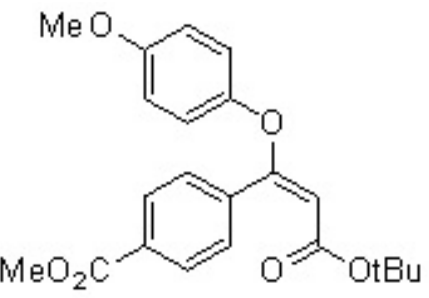

(E) -2

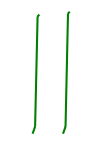


13C NMR (176 MHz, Chloroform-d)

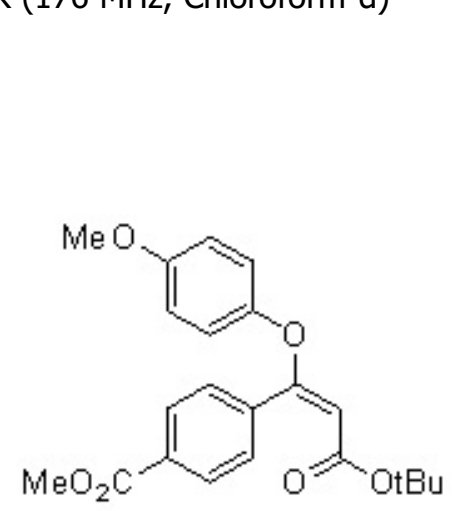

(E) -2

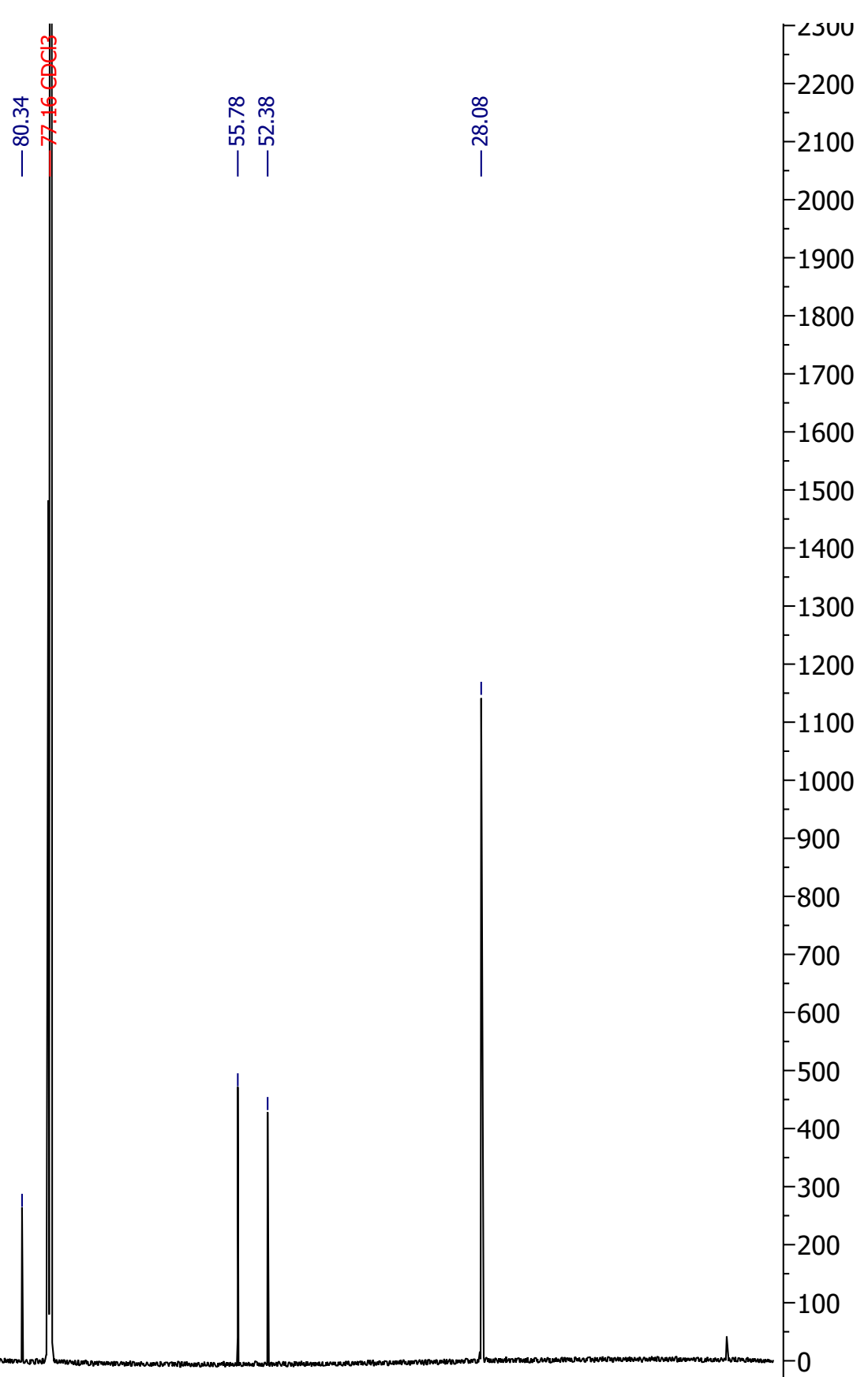




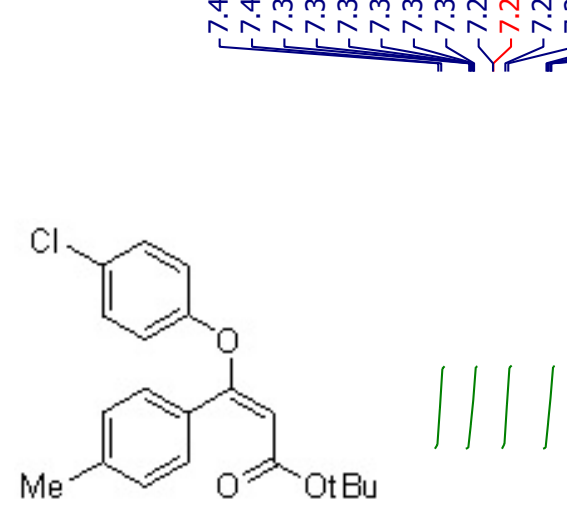

(E) -3 
13C NMR (176 MHz, Chloroform-d)

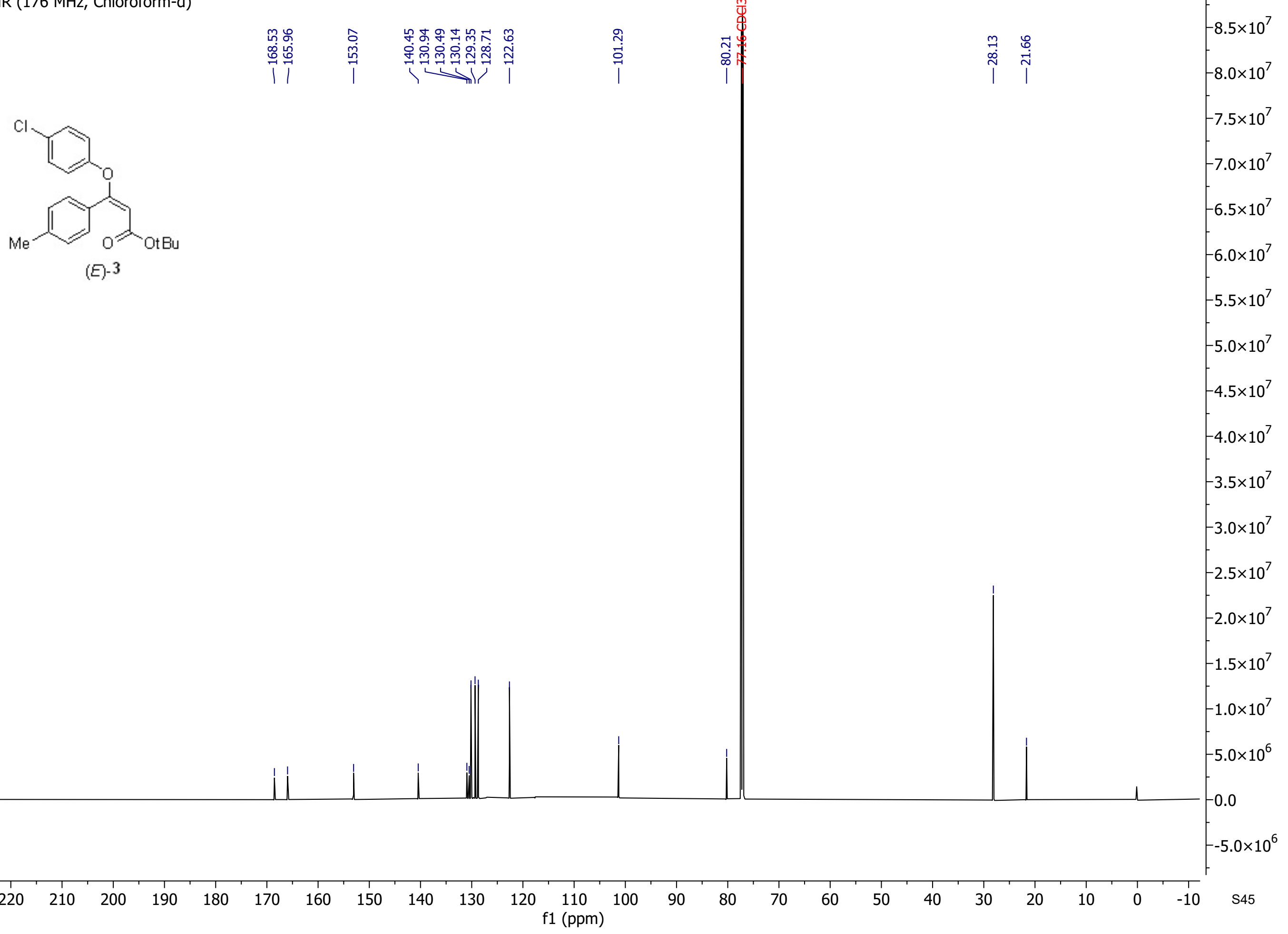


1H NMR ( 700 MHz, Chloroform-d)

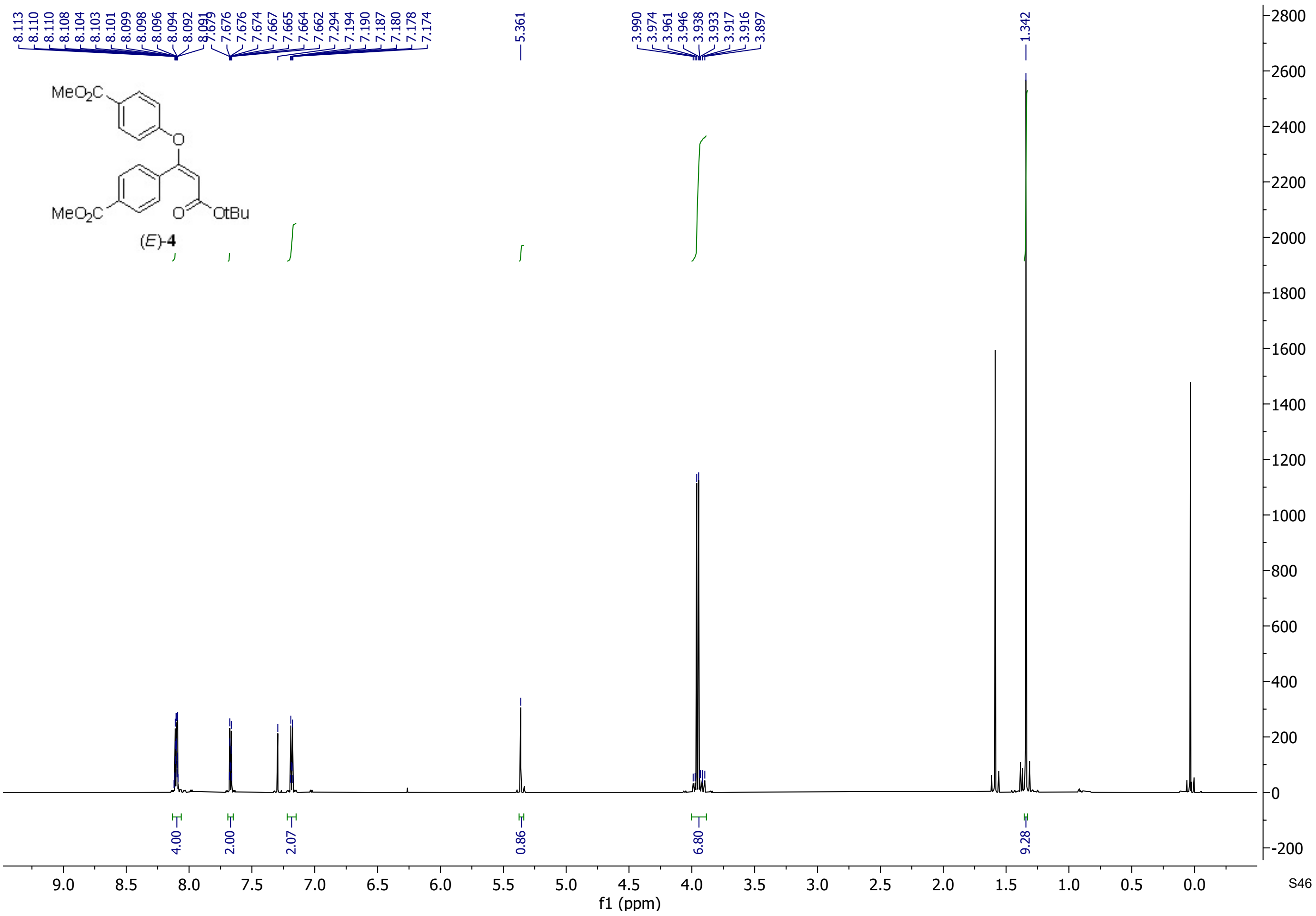


13C NMR (176 MHz, Chloroform-d)

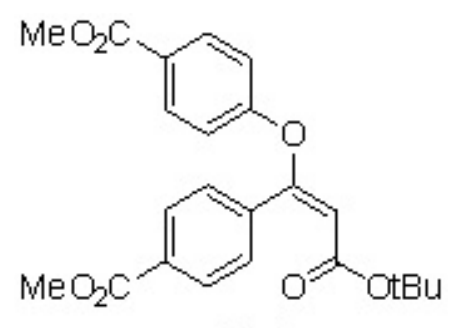

$(E)-4$ 
<smiles>CCOC(=O)/C=C(/Oc1ccc(OC)cc1)c1ccc(C(C)=O)cc1</smiles>

$(E)-5$ 
13C NMR (176 MHz, Chloroform-d)

$\mathrm{MeO}$

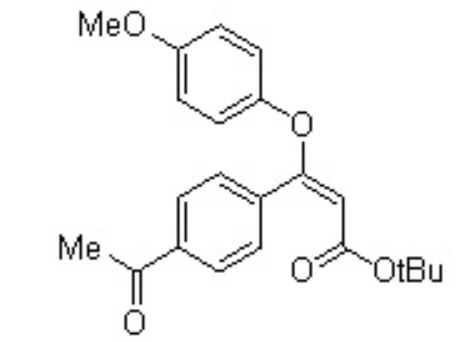

(E)-5

ll

(E)- 5

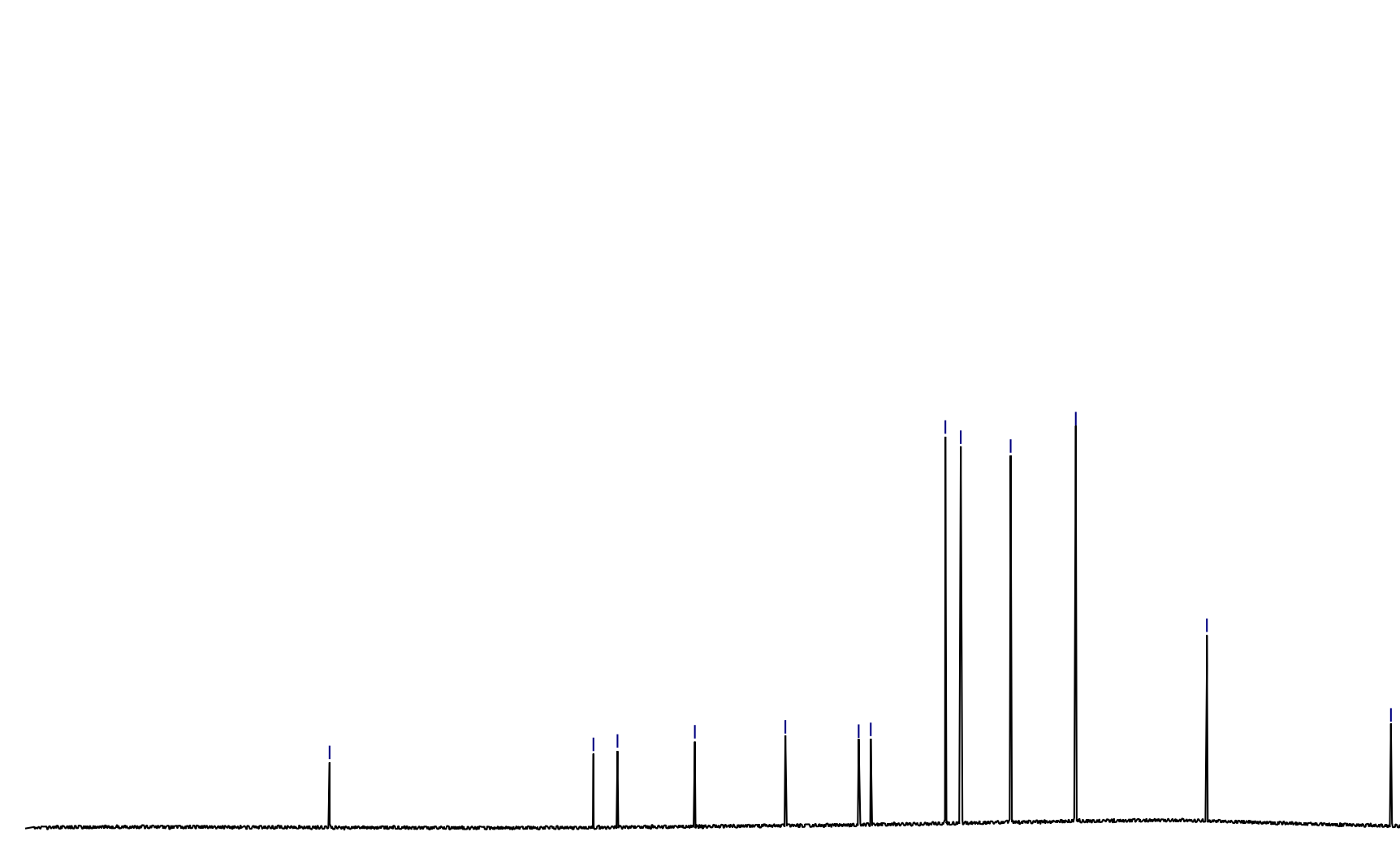


<smiles>CCOC(=O)/C=C(/Oc1ccc(OC)cc1)c1ccc(Br)cc1</smiles>

(E)-6
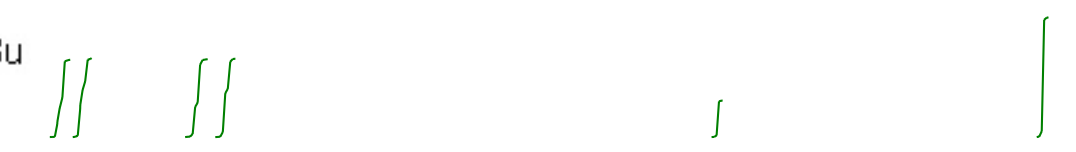
13C NMR (101 MHz, Chloroform-d)

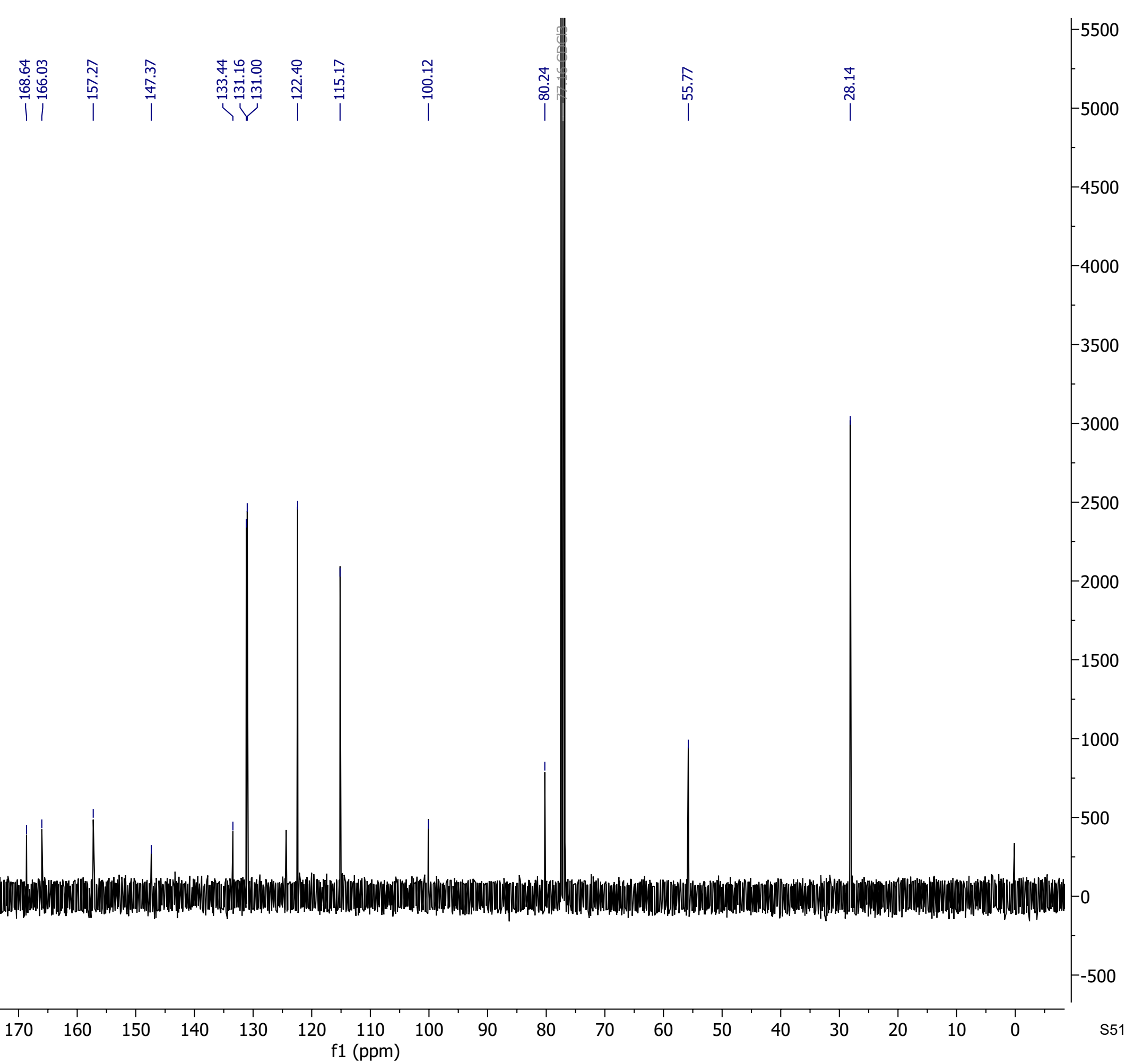




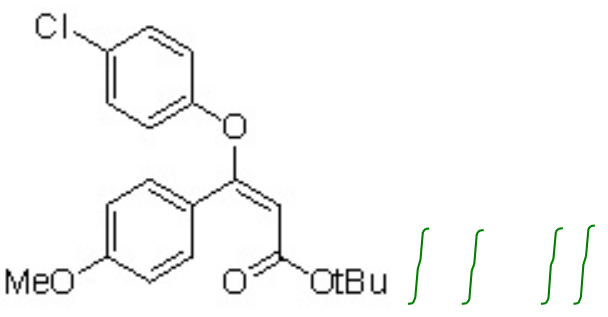

(E) -7

$-45000$

$-40000$

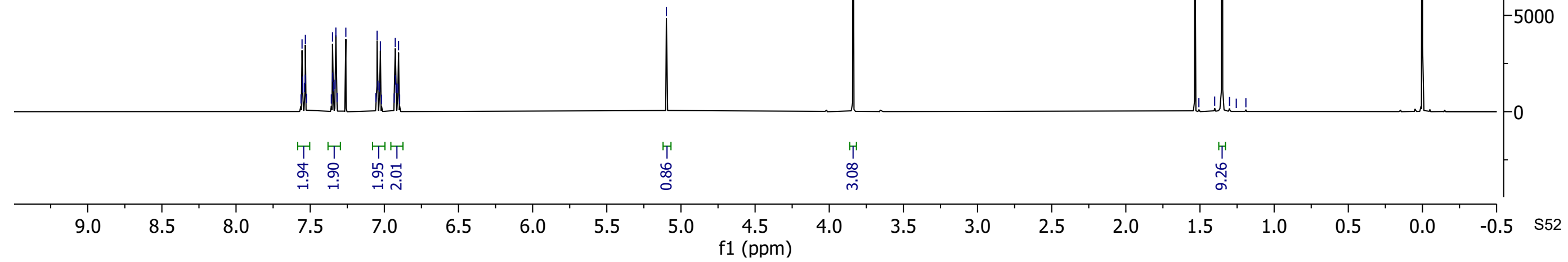


13C NMR (101 MHz, Chloroform-d)
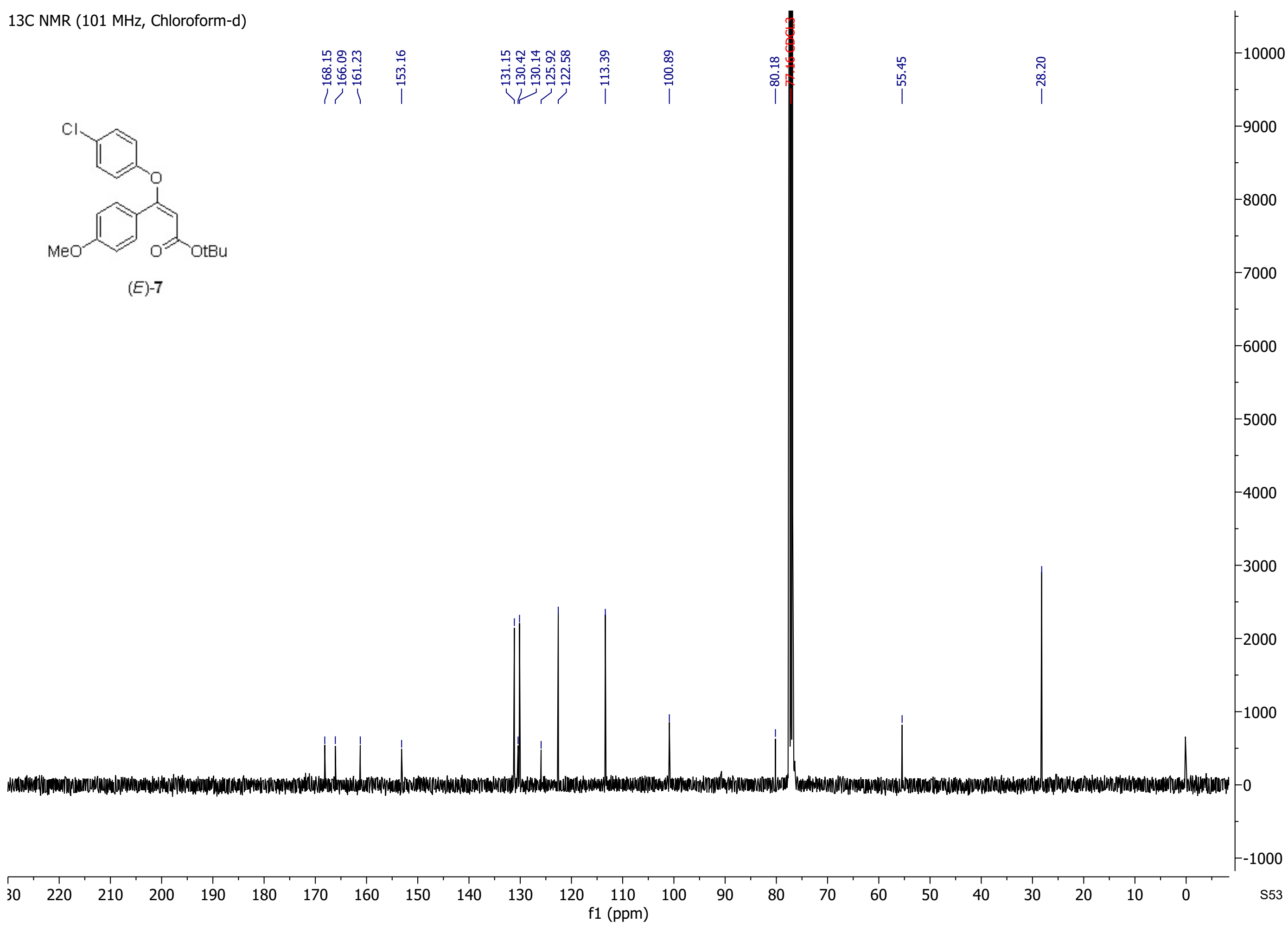


\section{H NMR (400 MHz, Chloroform-d)}

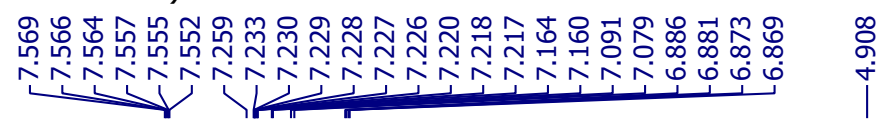

$\mathrm{MeO}$<smiles>BrC1=CCC=CC1</smiles>
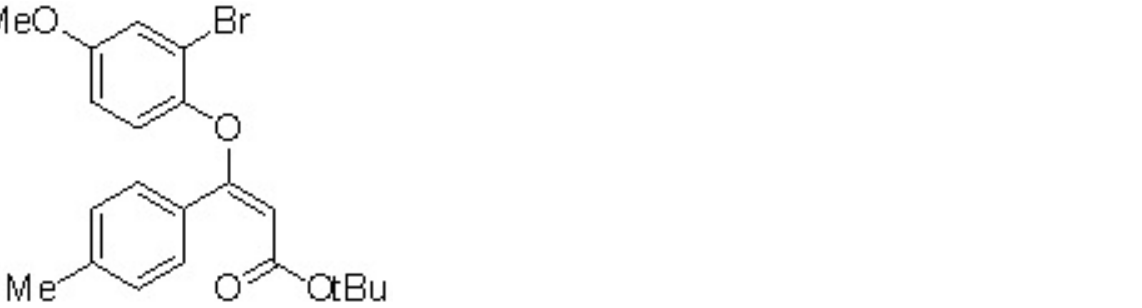

(E)-8

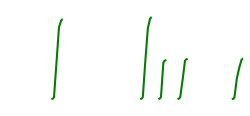




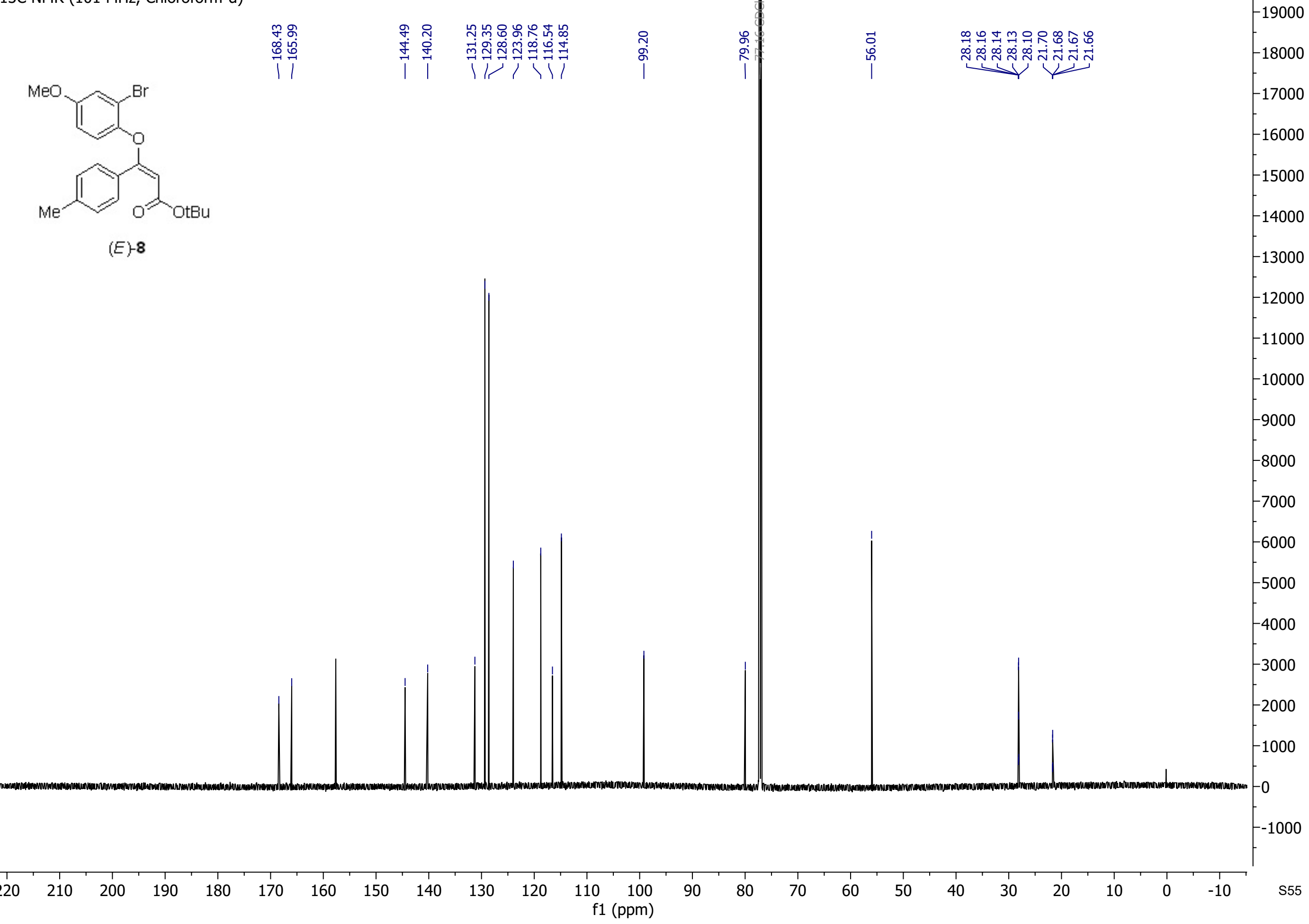




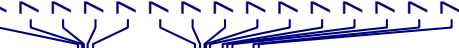

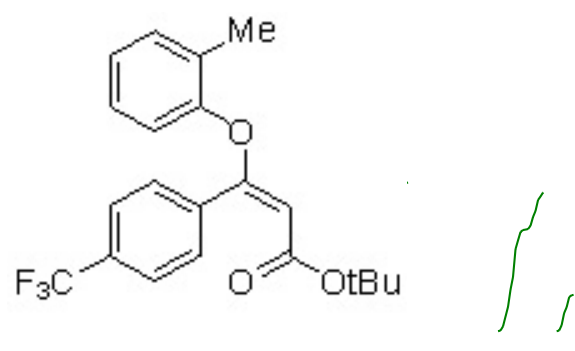

(E)-9

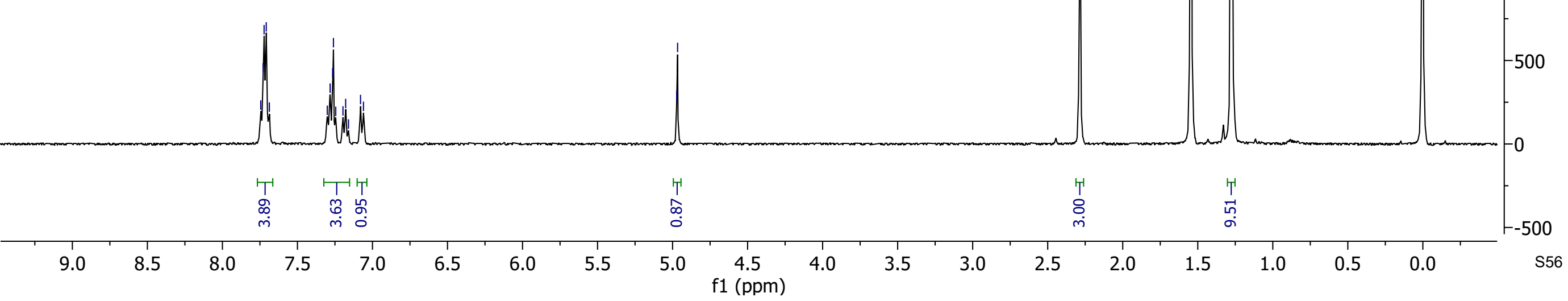




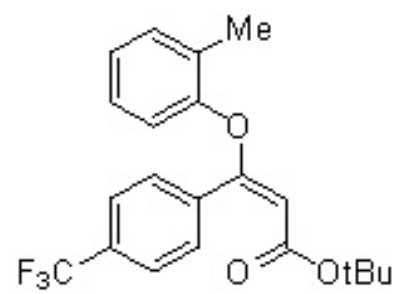

(E)- 9

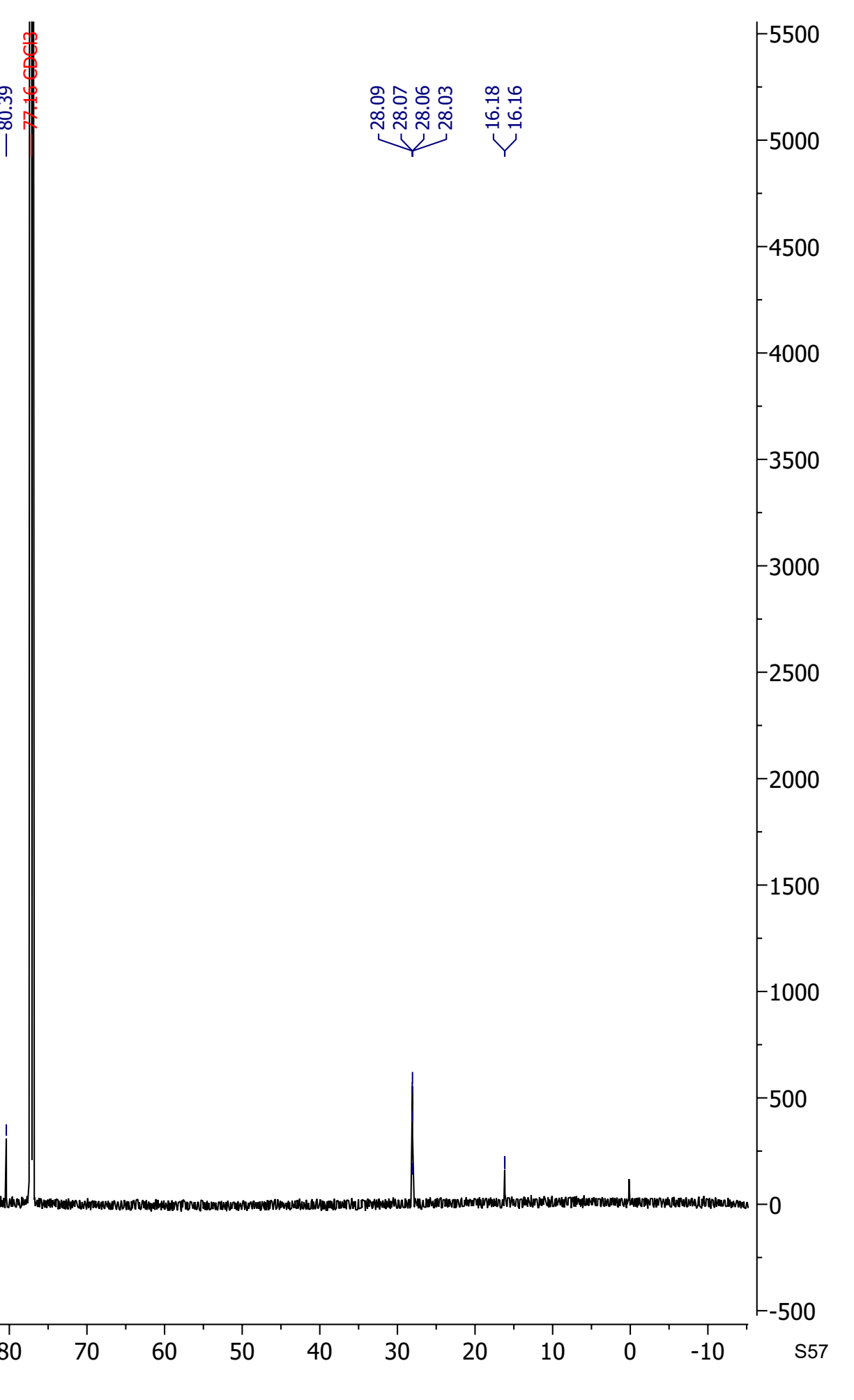


<smiles>CCCOC(=O)/C=C(/Oc1ccccc1C)c1ccc(C(F)(F)F)cc1</smiles>

(E)-9 


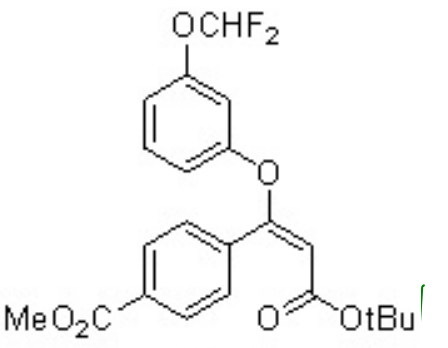

(E) -10
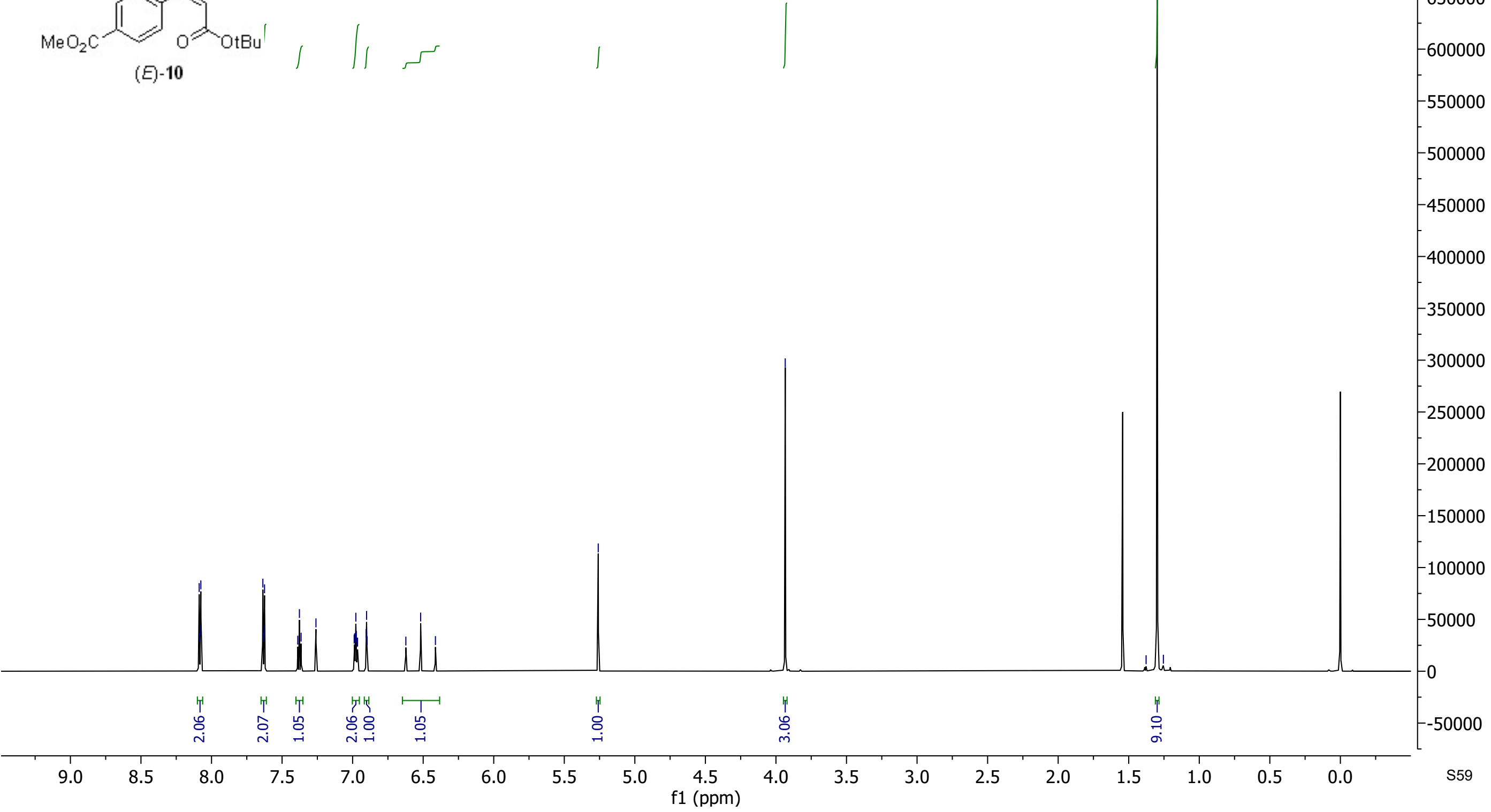


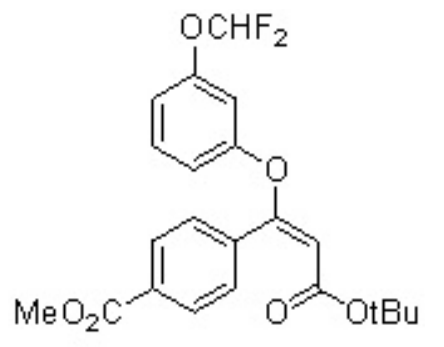

$(E)-10$ 


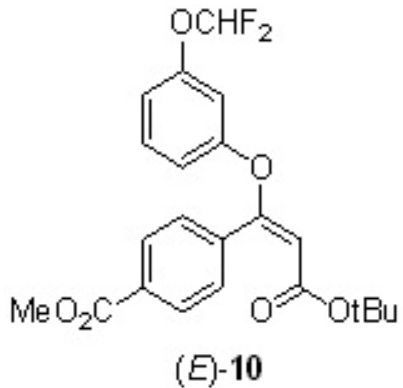




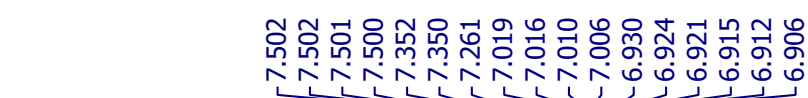

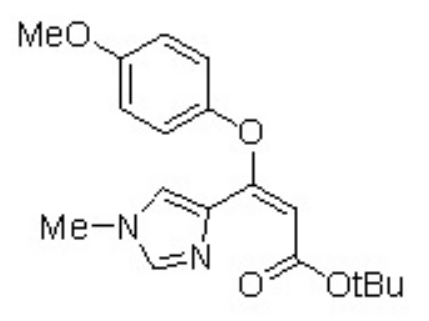

(E)-11 
13C NMR (176 MHz, Chloroform-d)

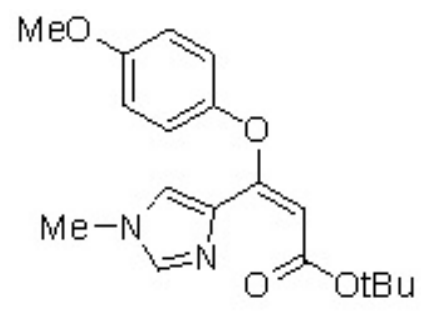

(E)-11 

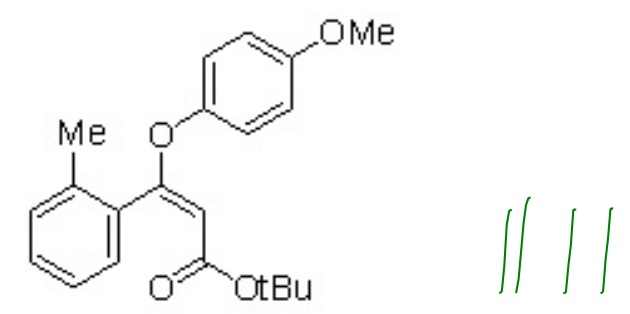

(E) 12

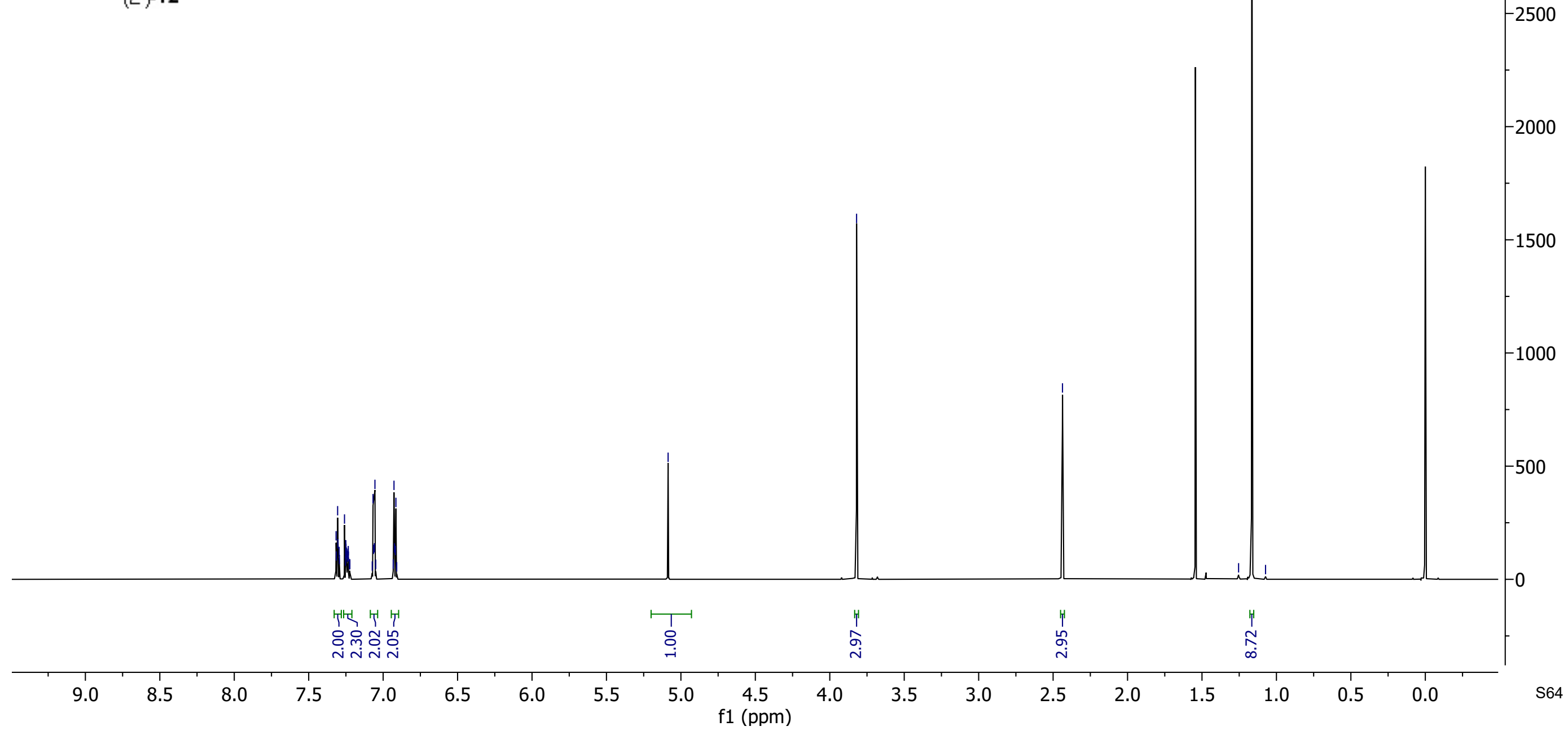


13C NMR (176 MHz, Chloroform-d)

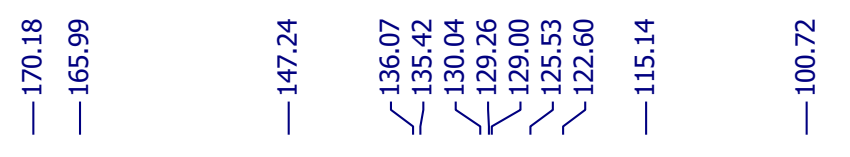

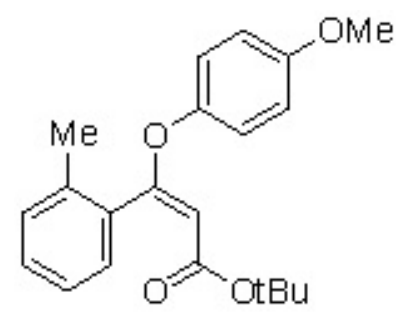

(E) 12 


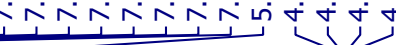
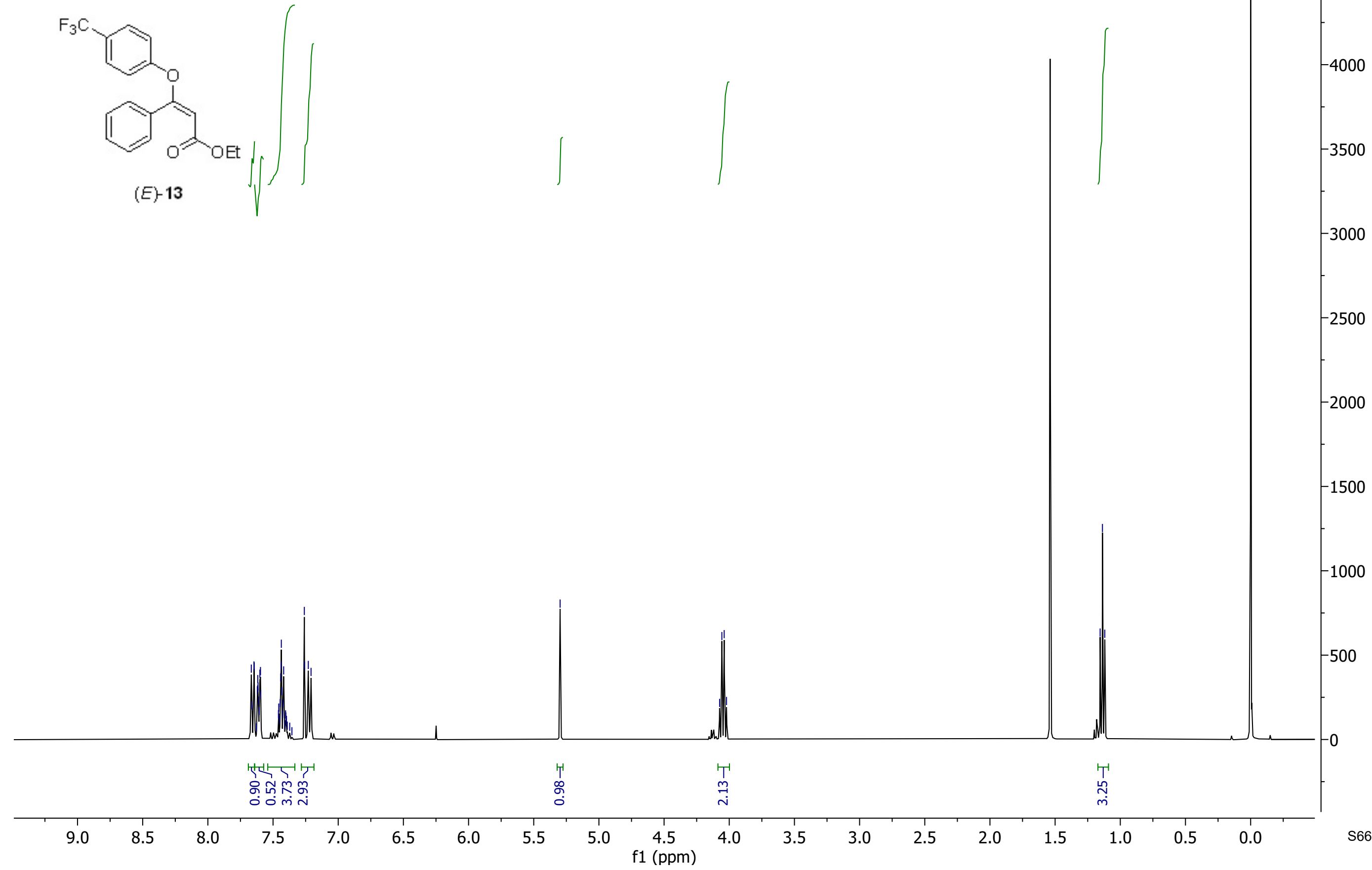
13C NMR (101 MHz, Chloroform-d)
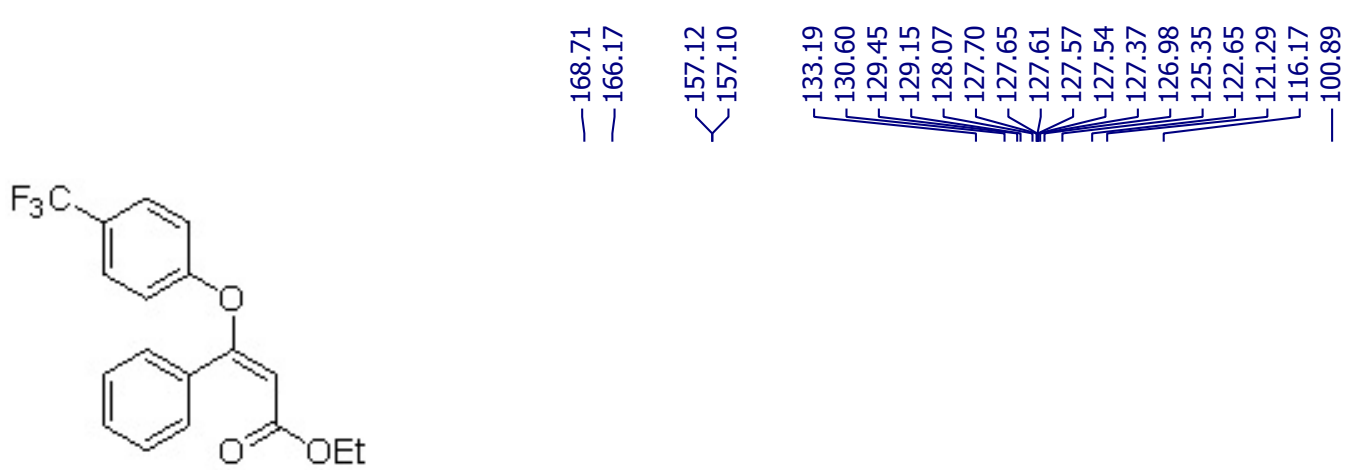

(E) 13

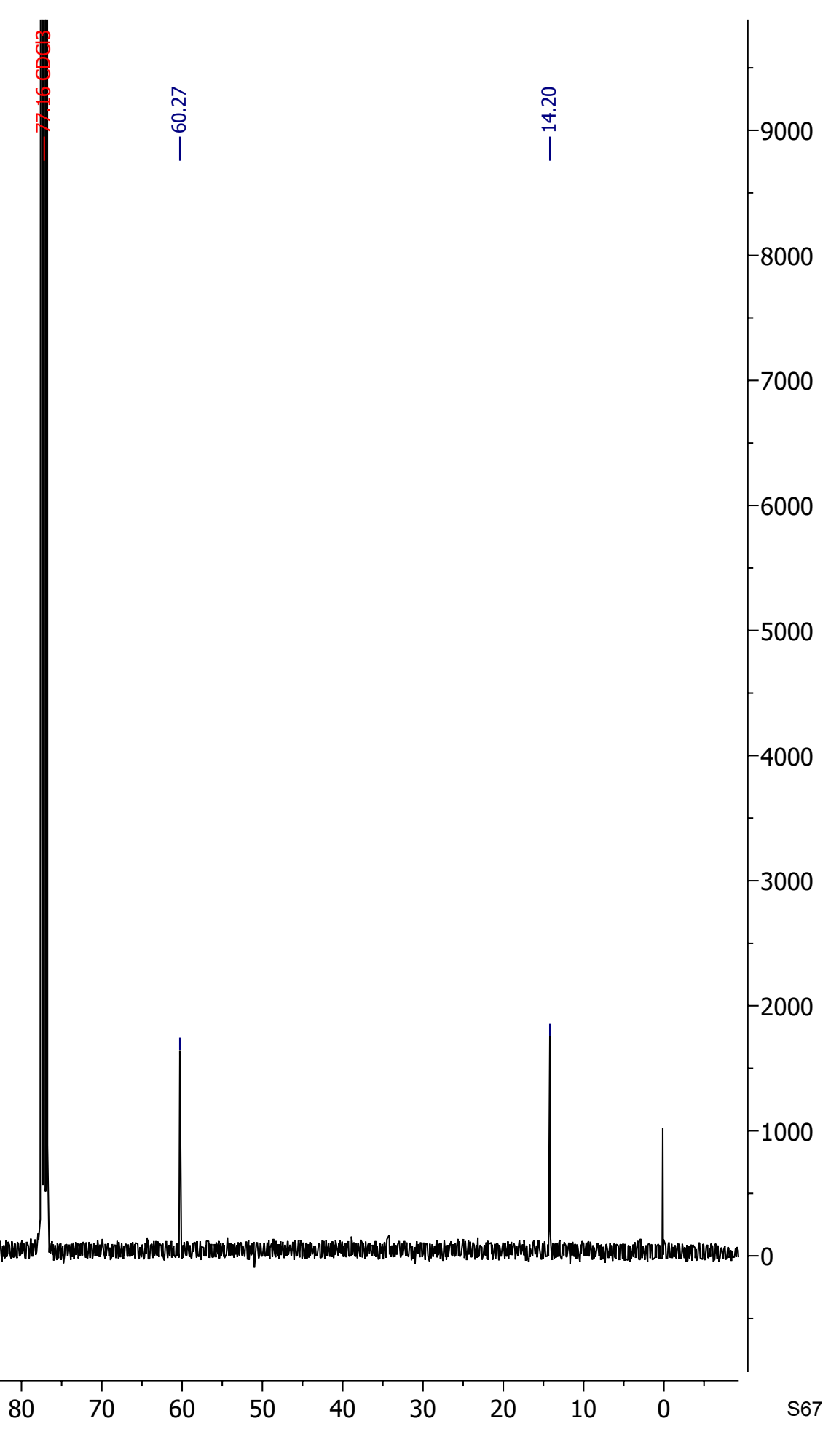




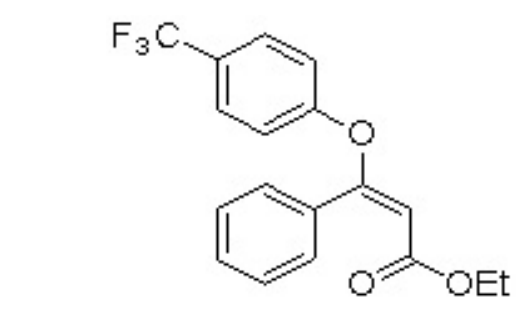

$-600$

(E)-13

$-500$

$-450$

$-400$

$-350$

$-300$

$-250$

$-200$

$-150$

$-100$

$-50$ 

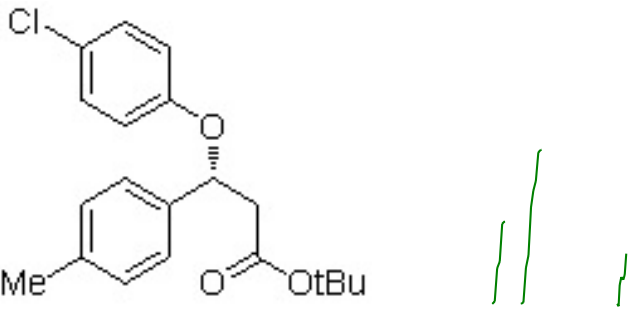

(R) 15

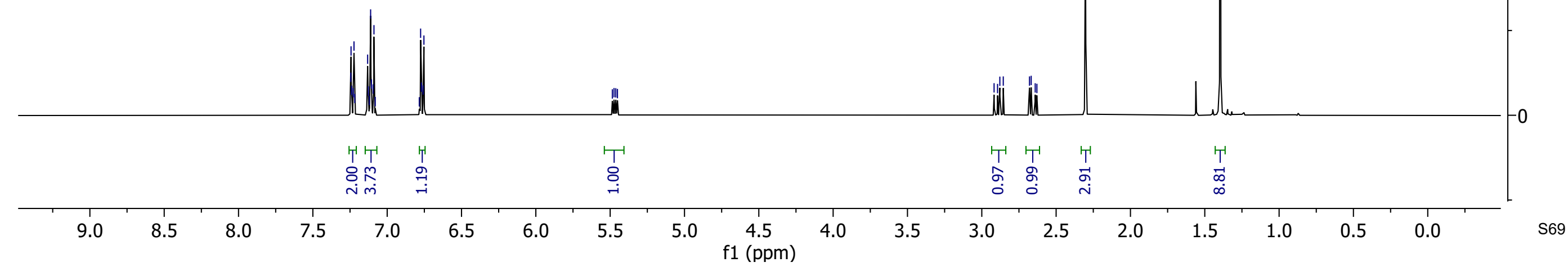




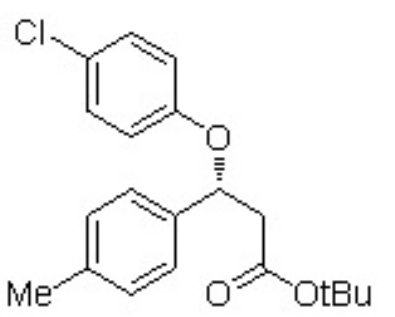


<smiles>CCOC(=O)C[C@H](Oc1ccc(C)cc1)c1ccc(OC)cc1</smiles>

(R) 16 


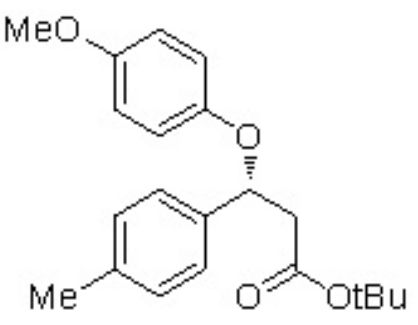

(R) 16

\section{OtBu}




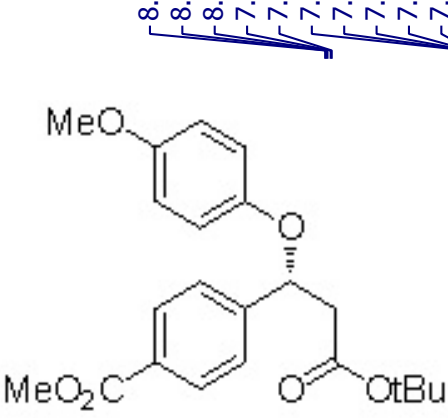

$(R)-17$

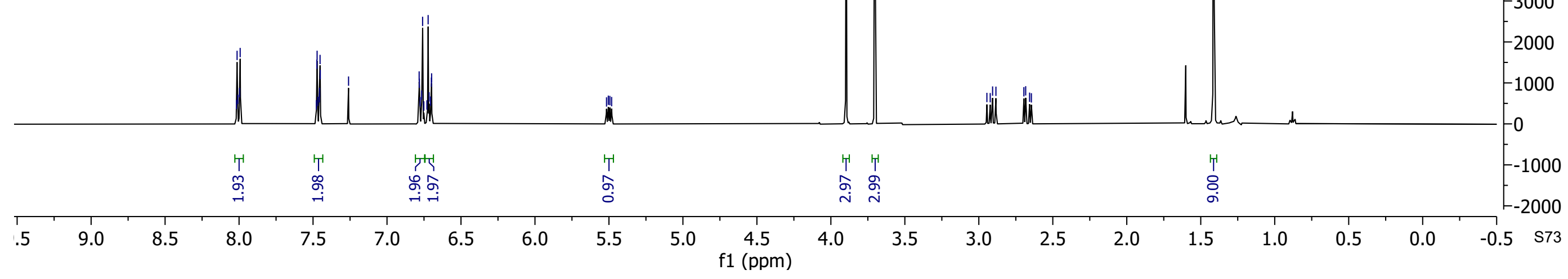


13C NMR (101 MHz, Chloroform-d)

쇼 की

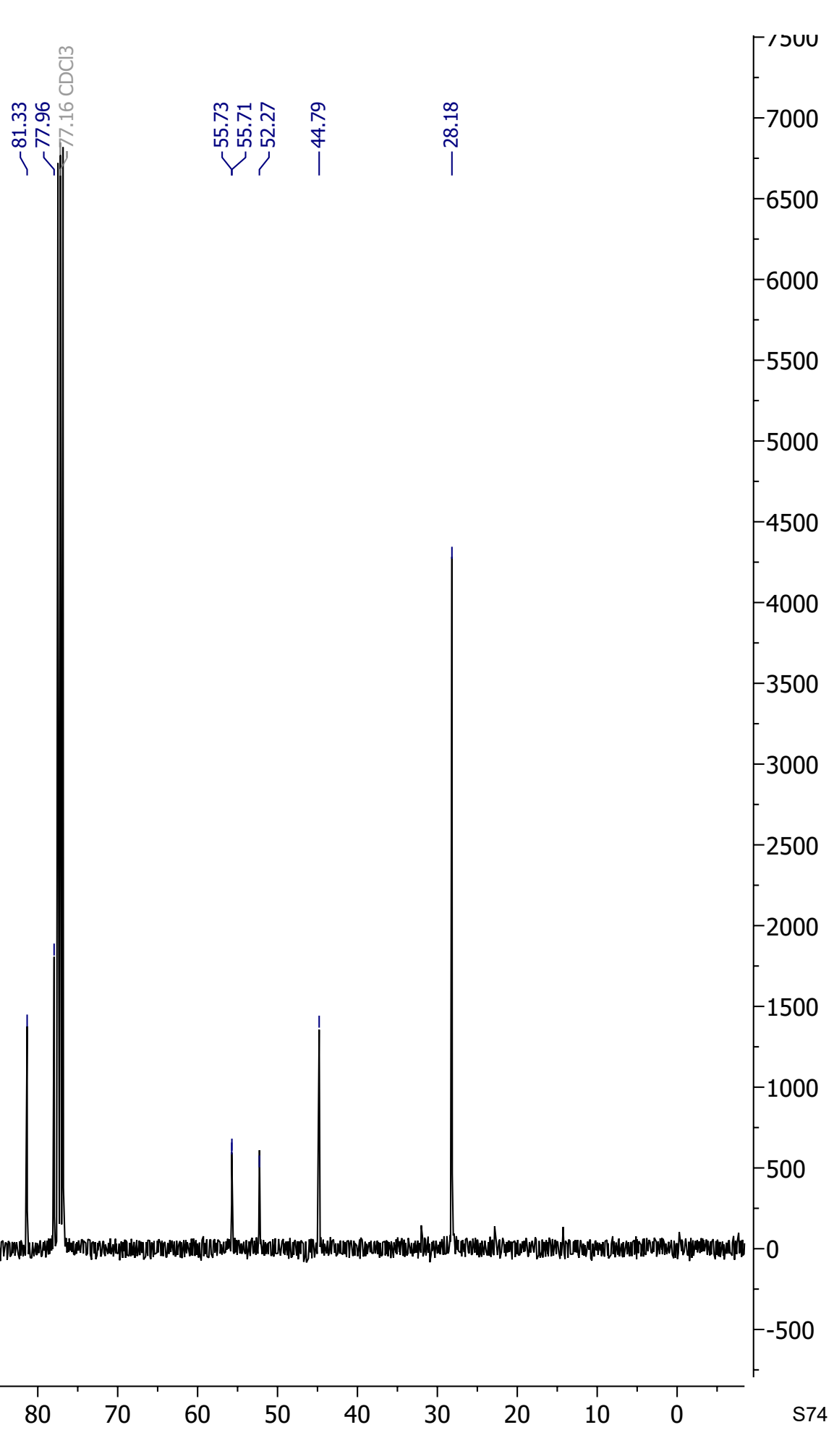




\section{H NMR (400 MHz, Chloroform-d)}

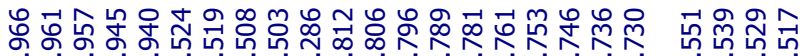
等
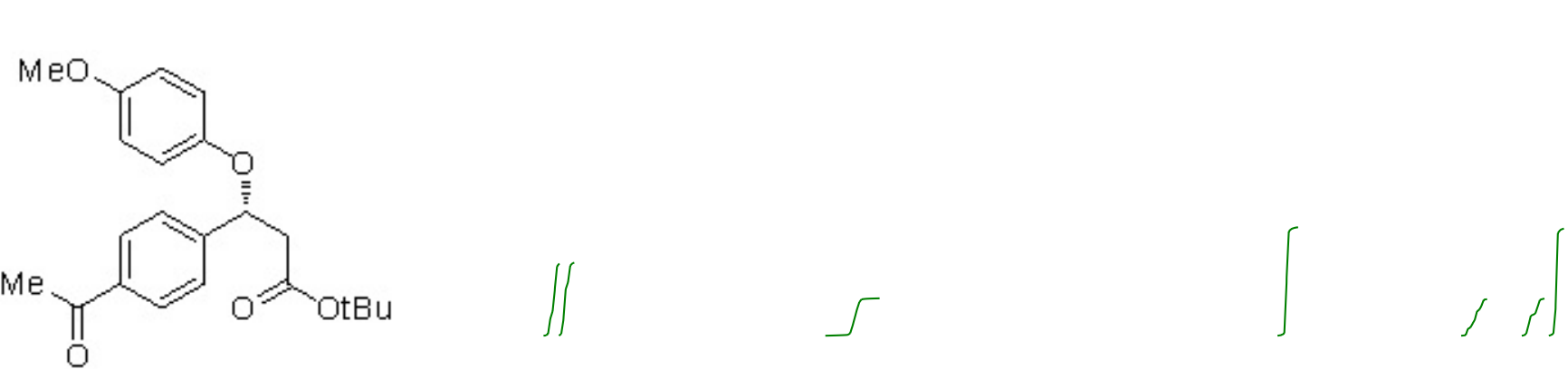

(R) 18 
13C NMR (101 MHz, Chloroform-d)

文

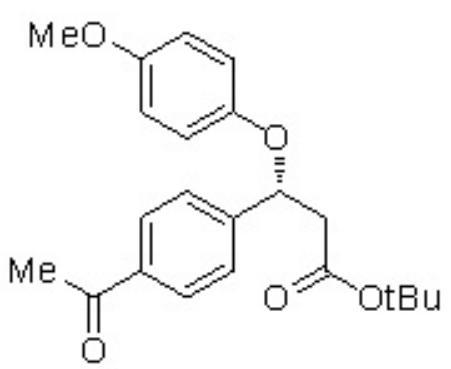

(R) 18

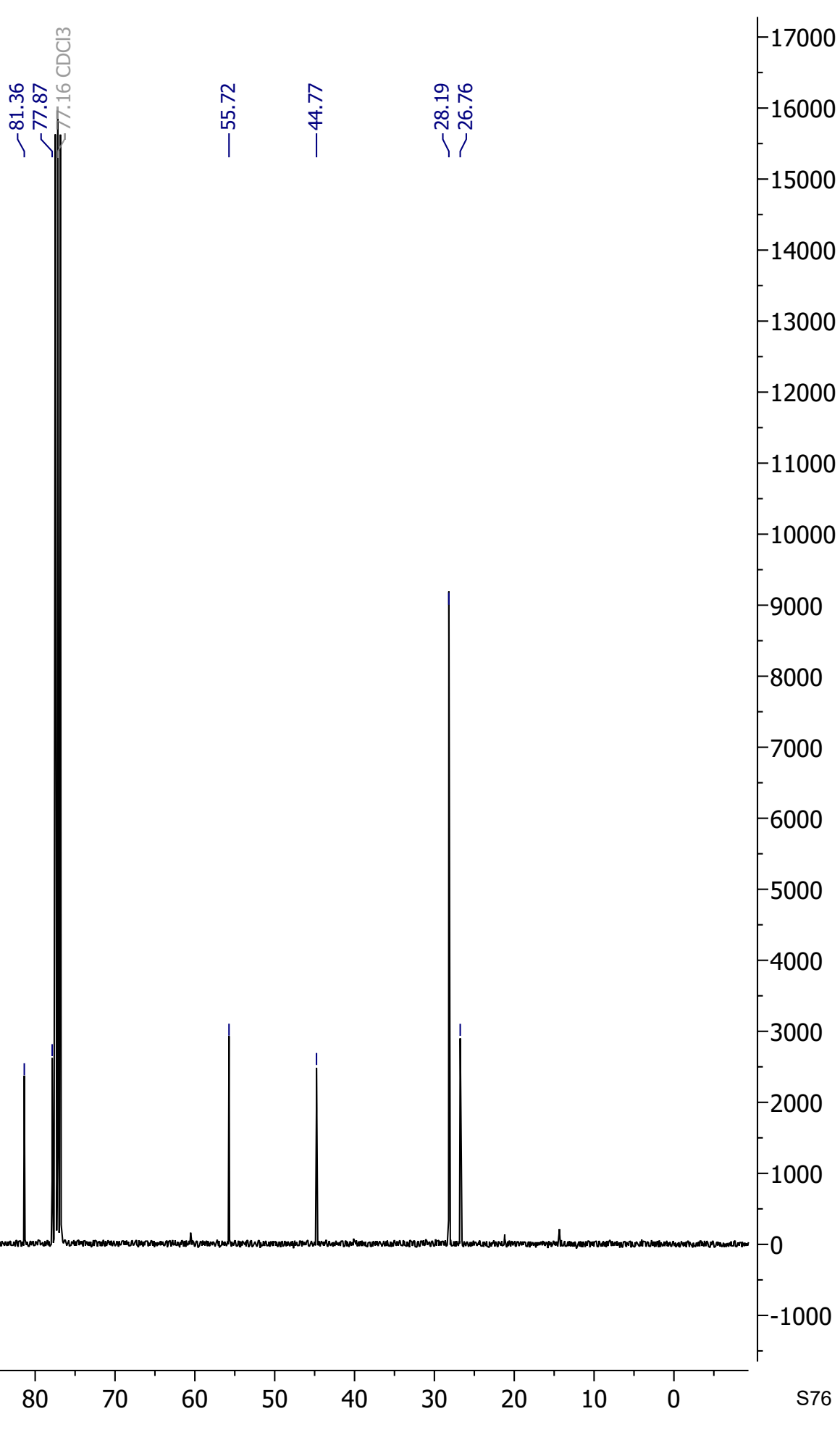


$1 \mathrm{H} \mathrm{NMR} \mathrm{(400} \mathrm{MHz,} \mathrm{Chloroform-d)}$

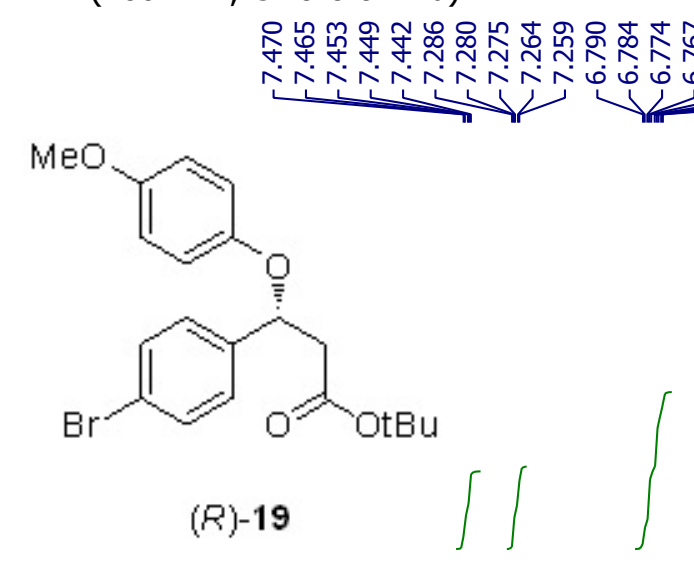

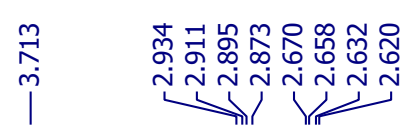

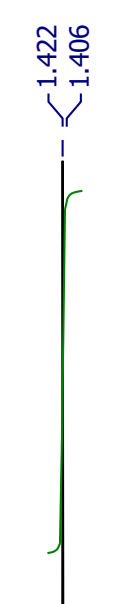

$-55000$

$-50000$

$-45000$

$-40000$

$-35000$

$-30000$

$-25000$

$-20000$

$-15000$

$-10000$

$-5000$

$-0$

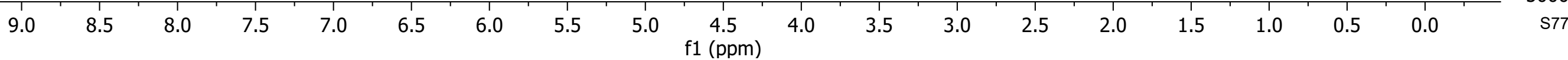


13C NMR (101 MHz, Chloroform-d)

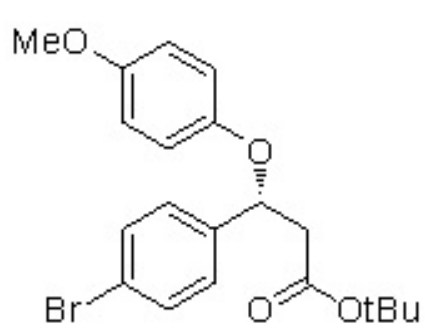

| ।

ill

$-75000$

70000

$-65000$

$-60000$

$-55000$

$(R)-19$

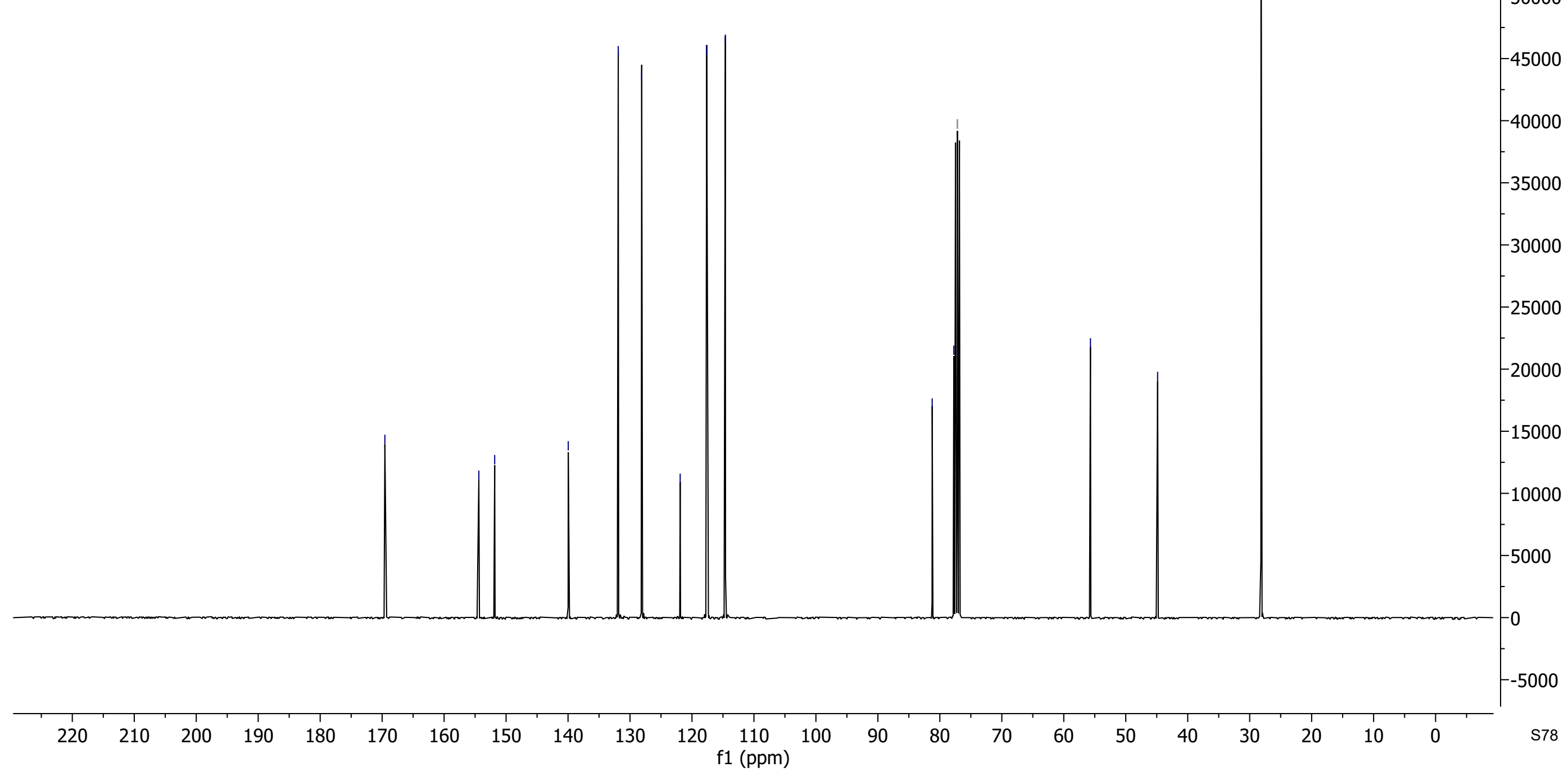




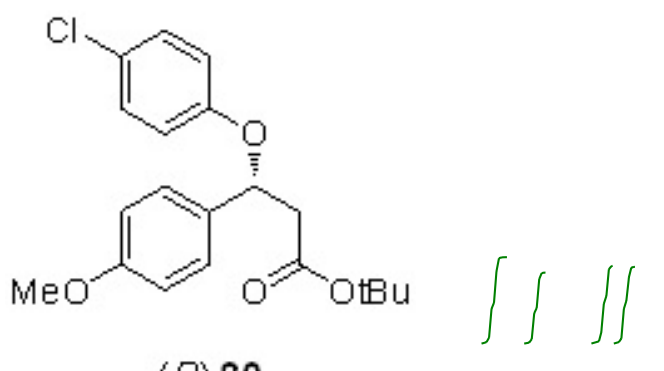

$(R)-20$
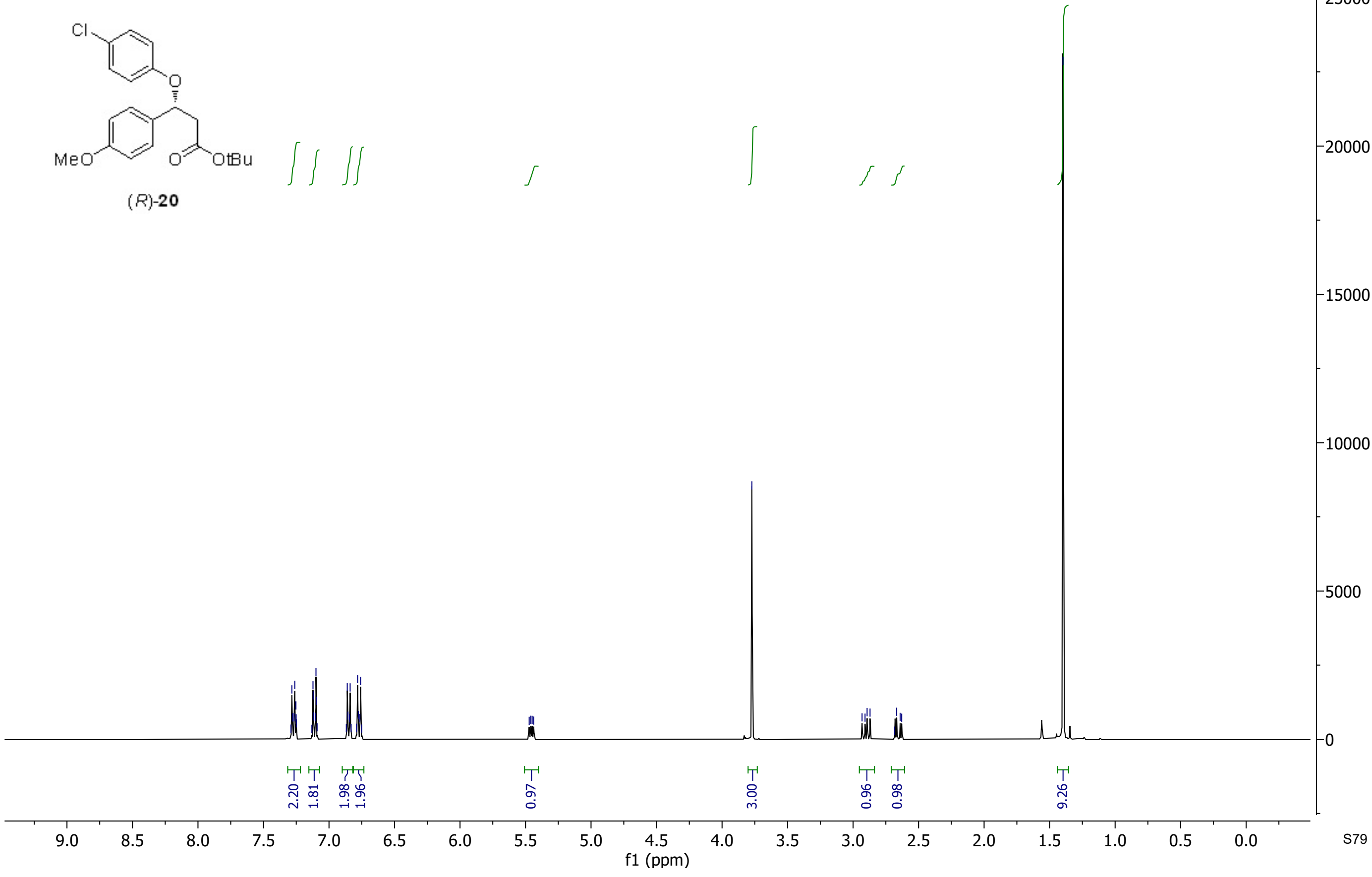


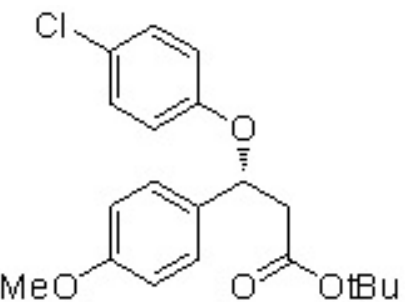

$(R)-20$ 
1H NMR (400 MHz, Chloroform-d)

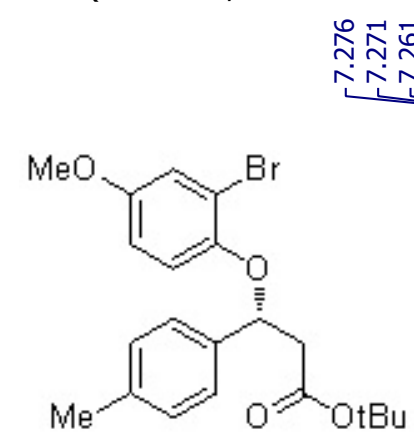

(R)-21

\section{尔喿守学}

กำ 난

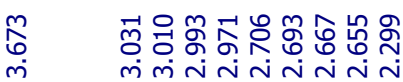


13C NMR (101 MHz, Chloroform-d)

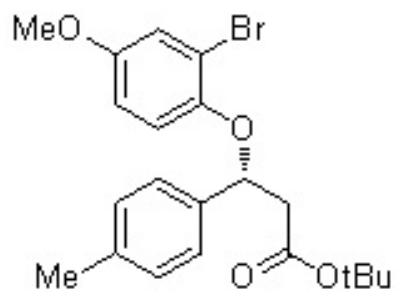

$(R)-21$

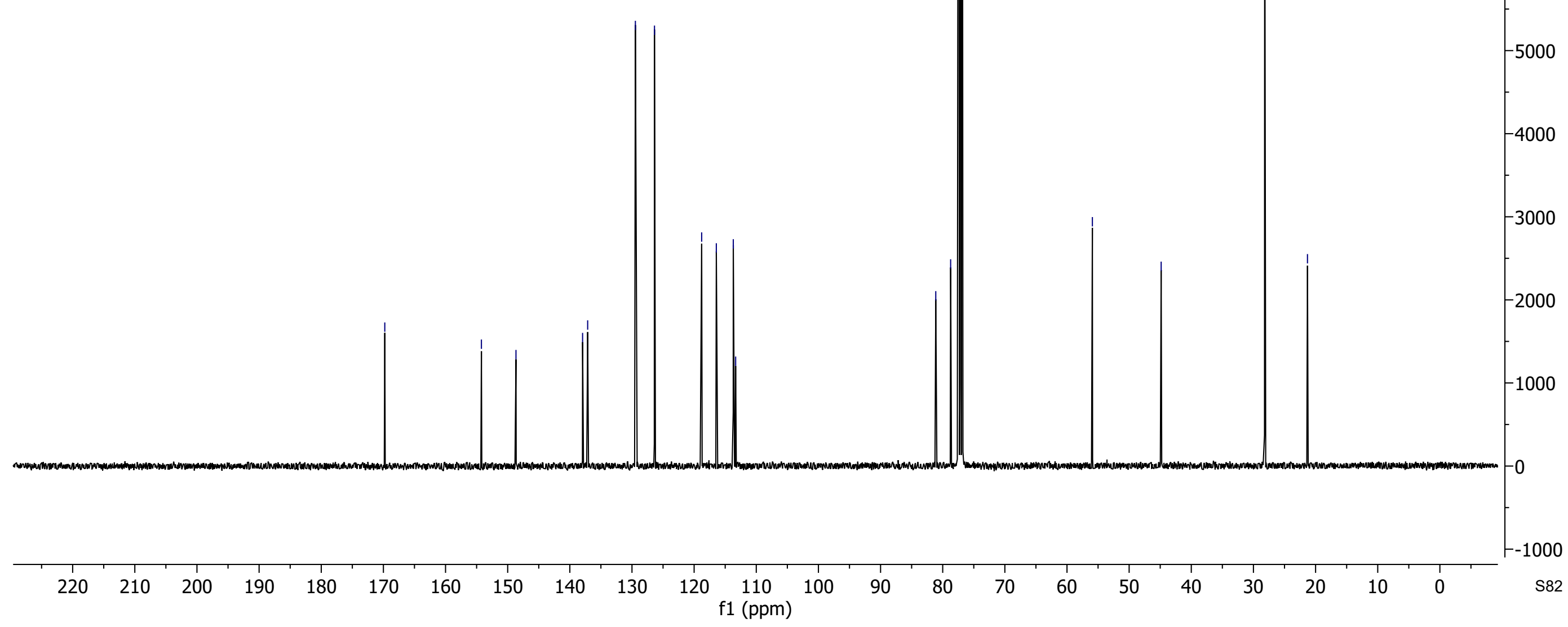


<smiles>CCCCOC(=O)CC(Oc1ccccc1C)c1ccc(C(F)(F)F)cc1</smiles>

(R)-22 
13C NMR (101 MHz, Chloroform-d)

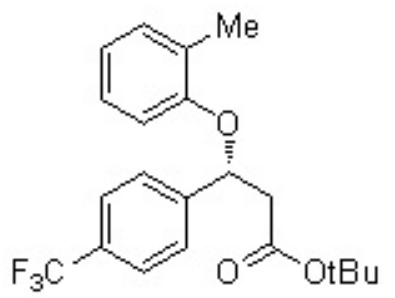

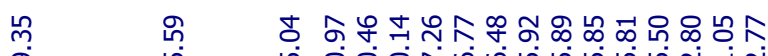

l

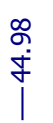

(R)-22 


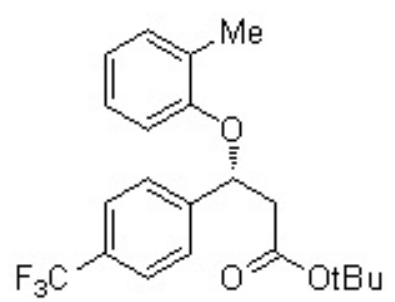

(R)-22 
1H NMR (400 MHz, Chloroform-d)

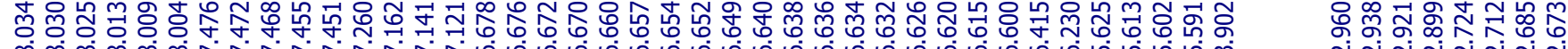<smiles>CCOC(=O)C[C@H](Oc1cccc(OC(F)F)c1)c1ccc(C(C)=O)cc1</smiles><smiles>C1=CCCCCC1</smiles>

(R) 23

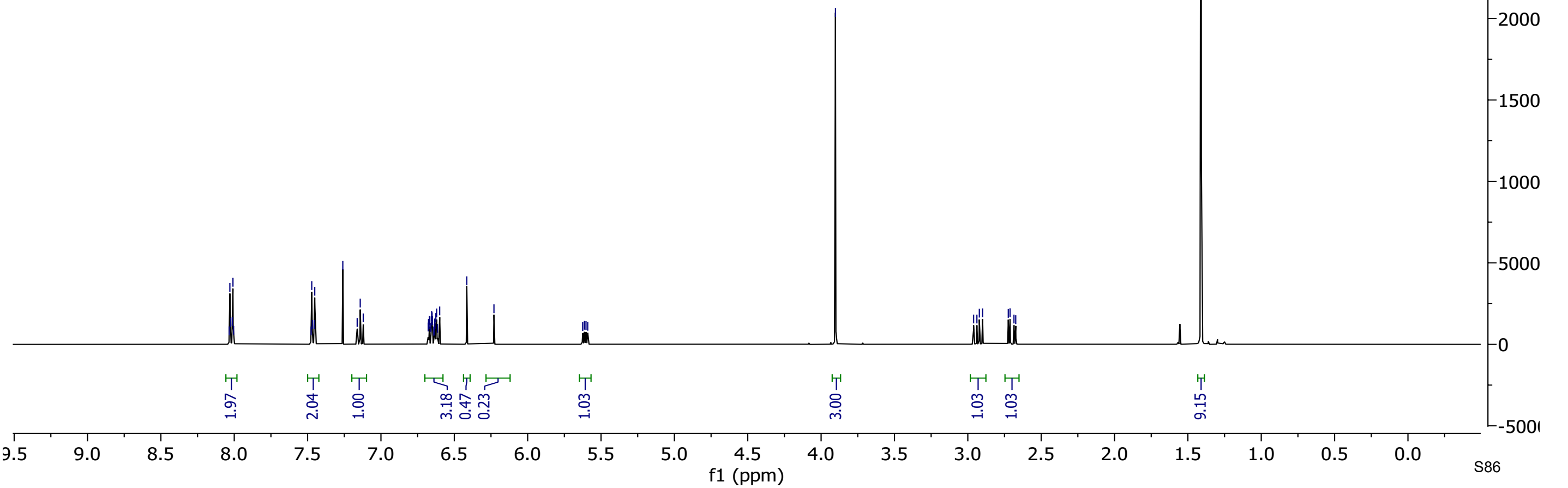


IIC MMR (101 MHz, Chloroform-d)<smiles>C1C[As]=[Ge]1</smiles>

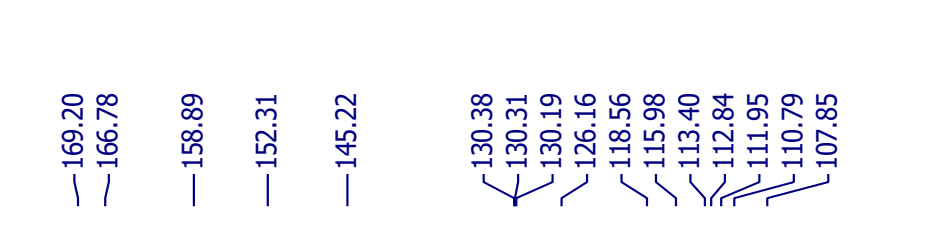




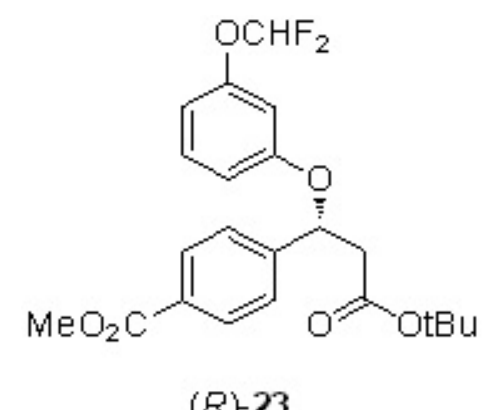

(R) 23

$-2500$ 
Compound 15, chiral separation:

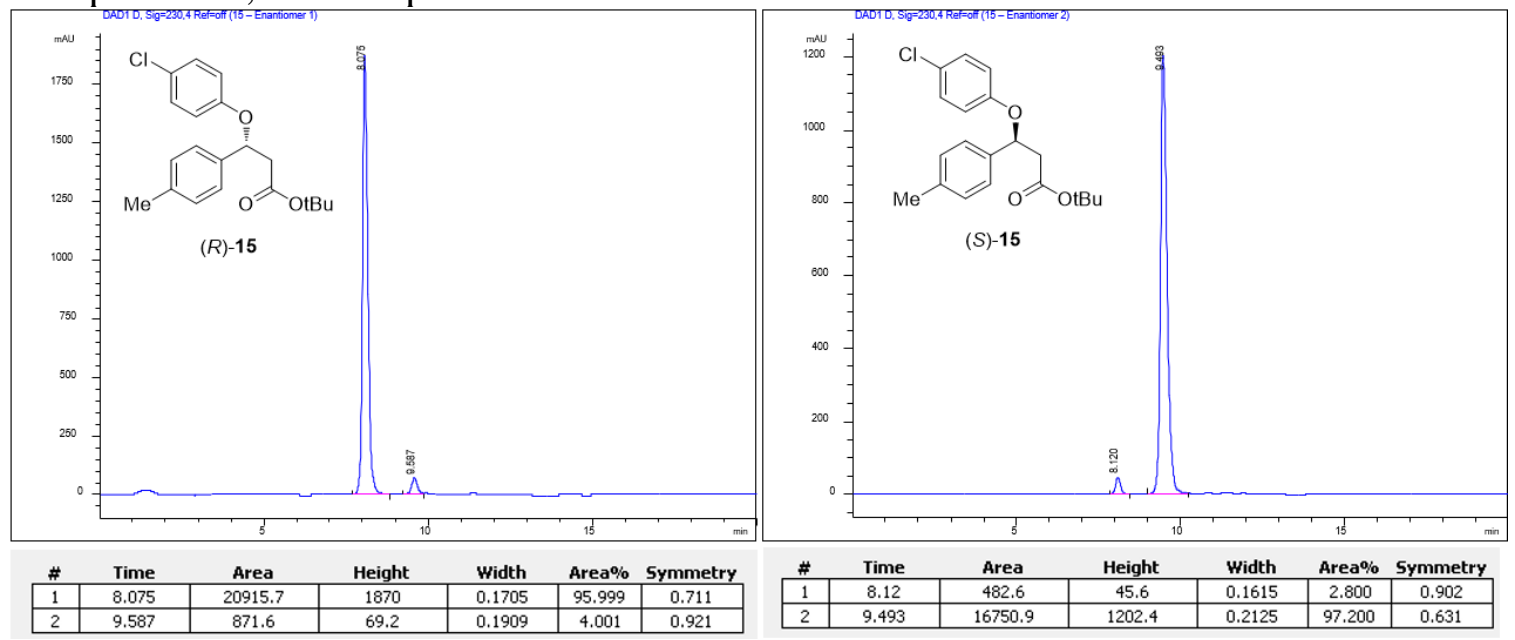

Compound 15, Figure 6:

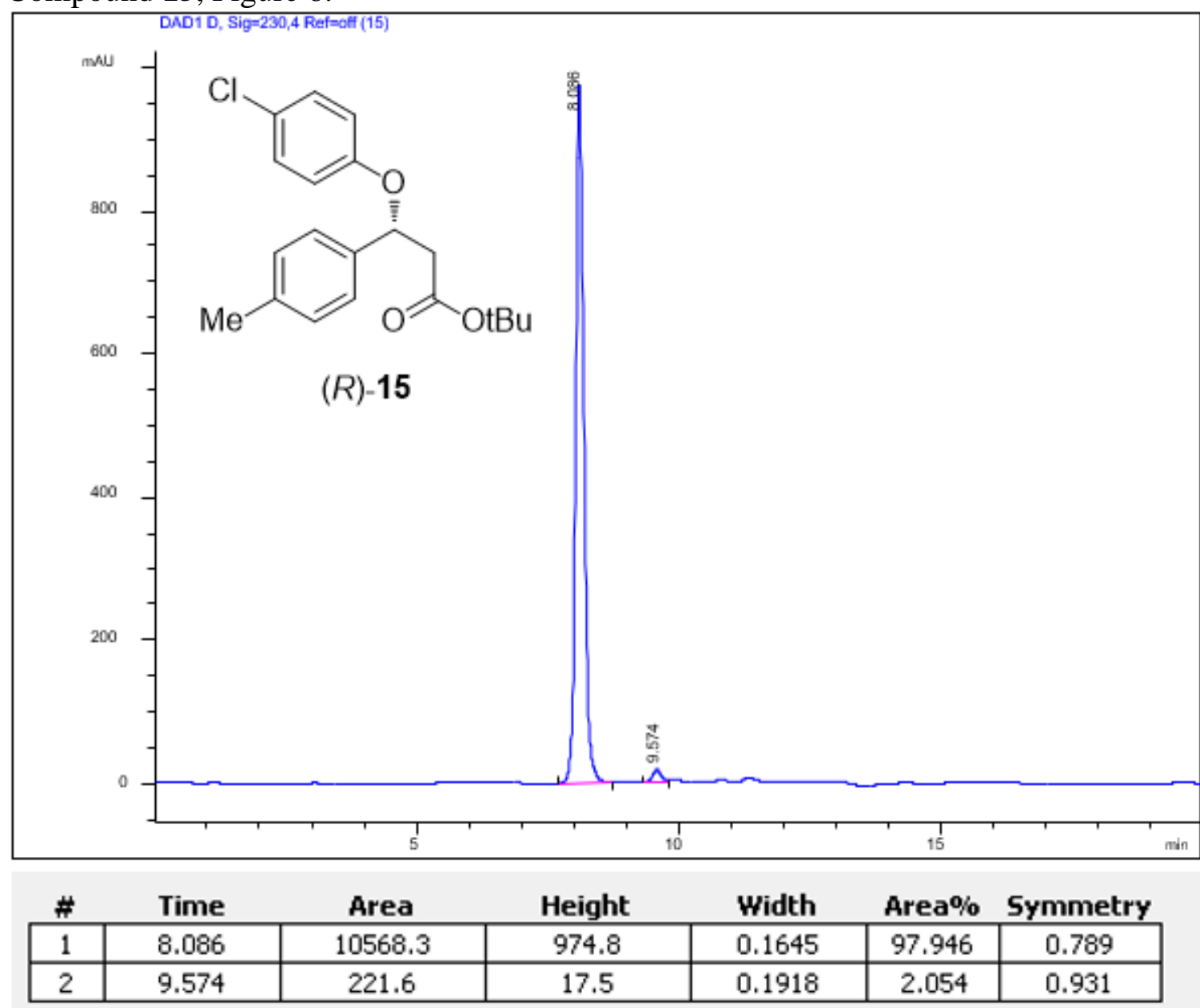


Compound 16, chiral separation:

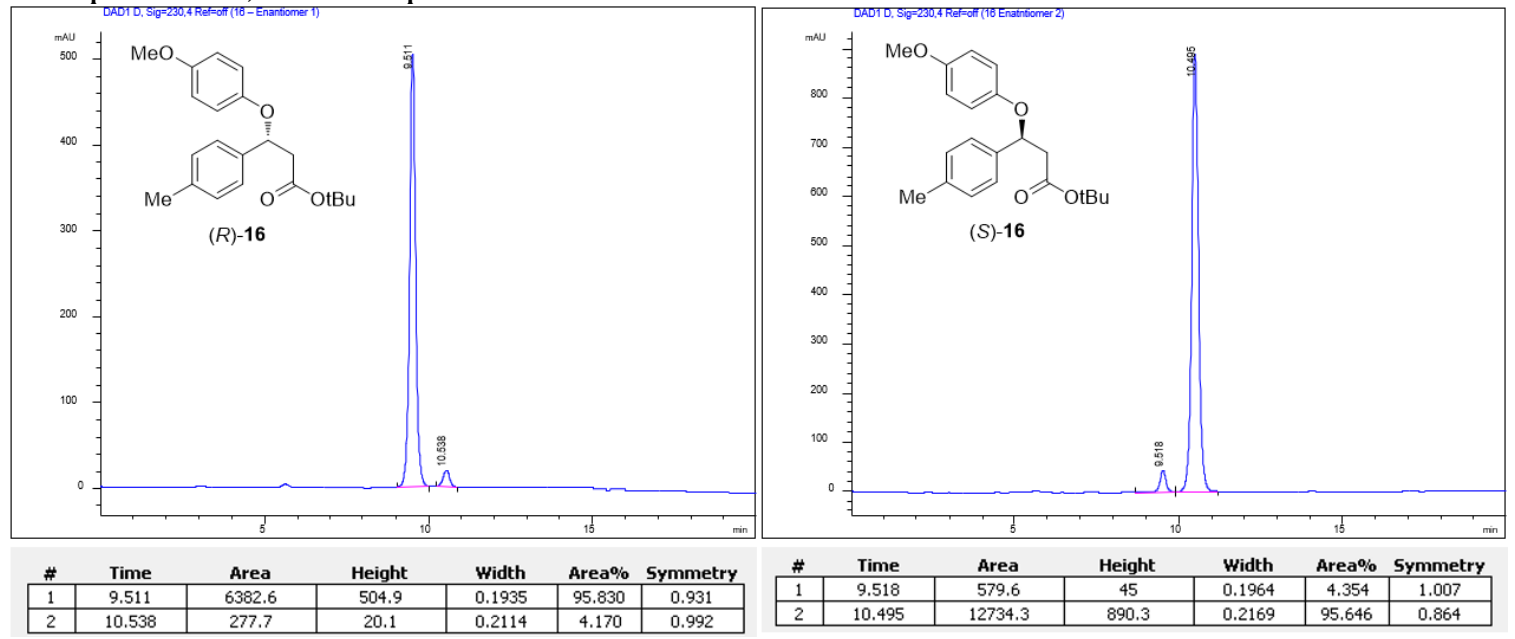

Compound 16, Figure 6:

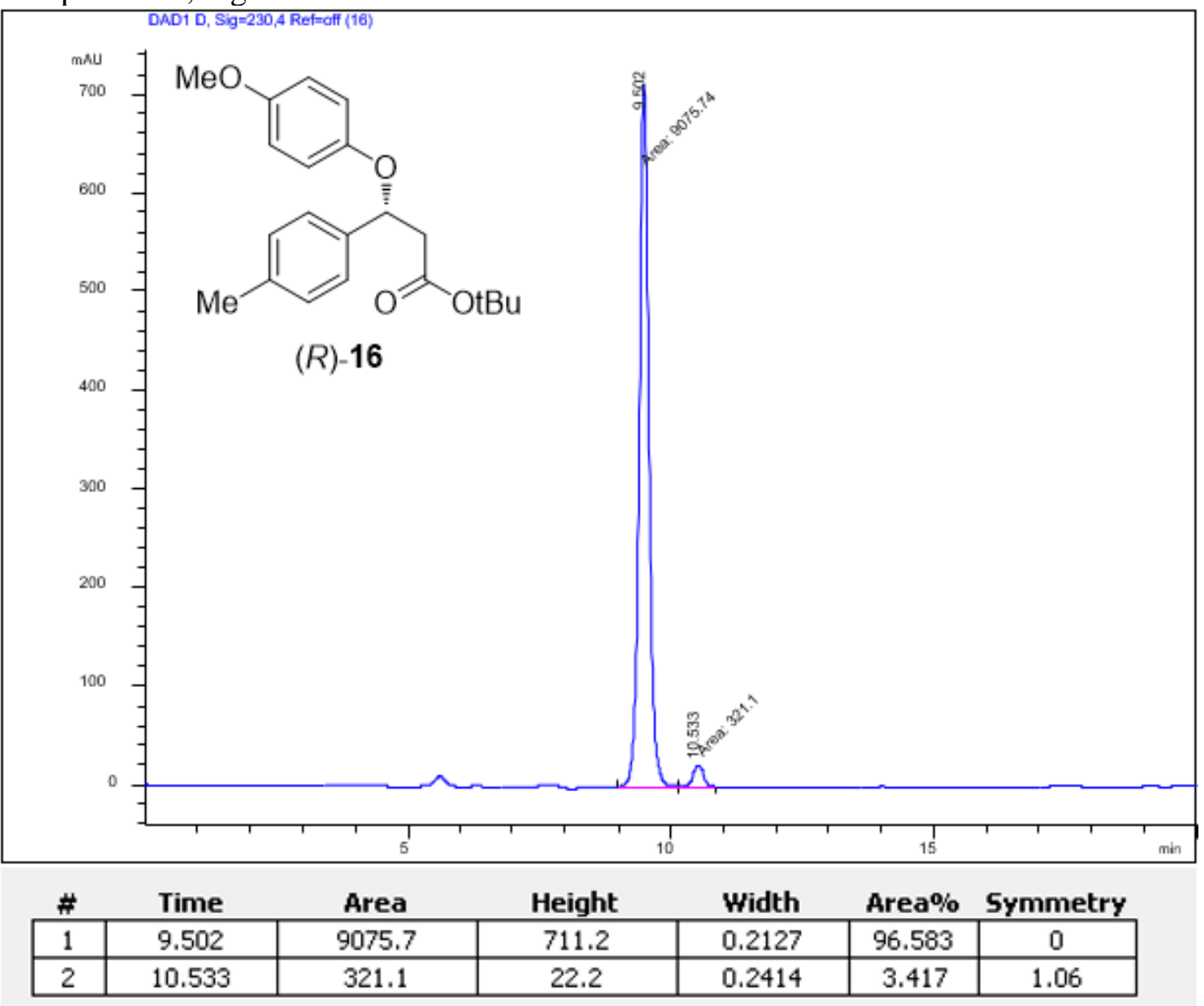


Compound 17, chiral separation:

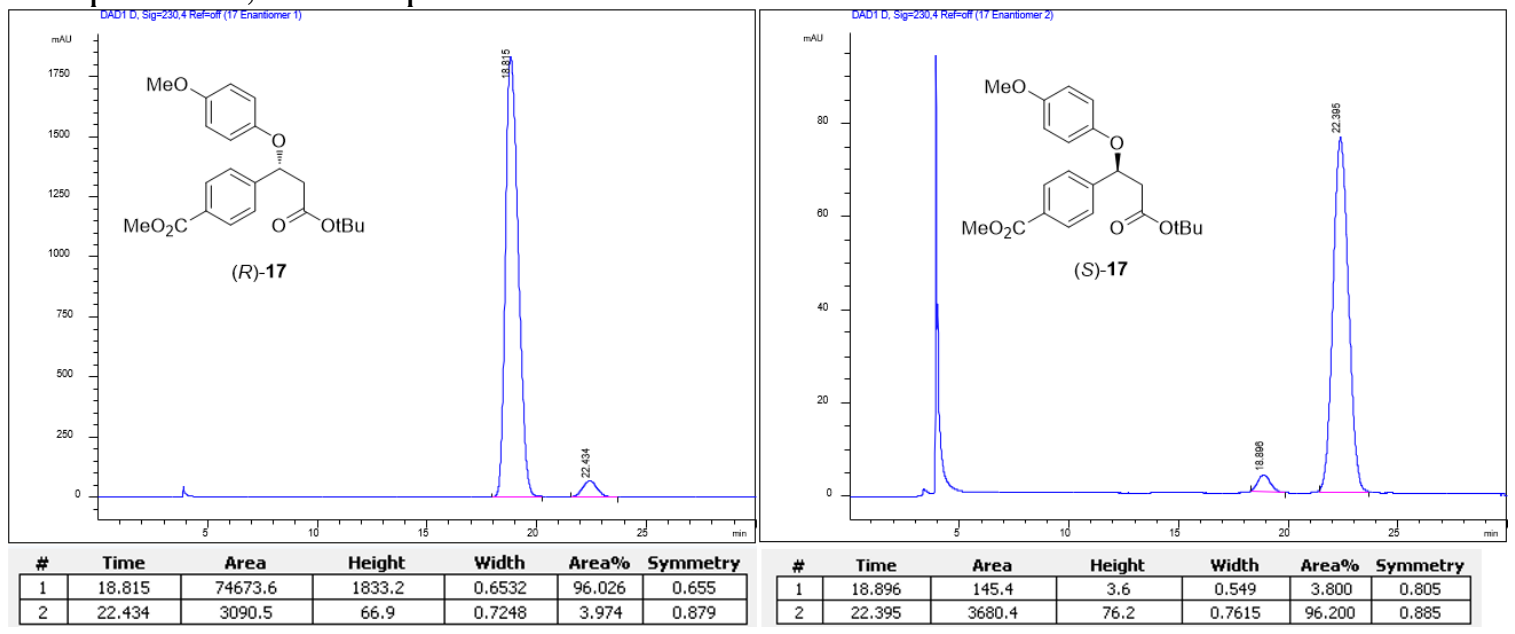

Compound 17, Figure 6:

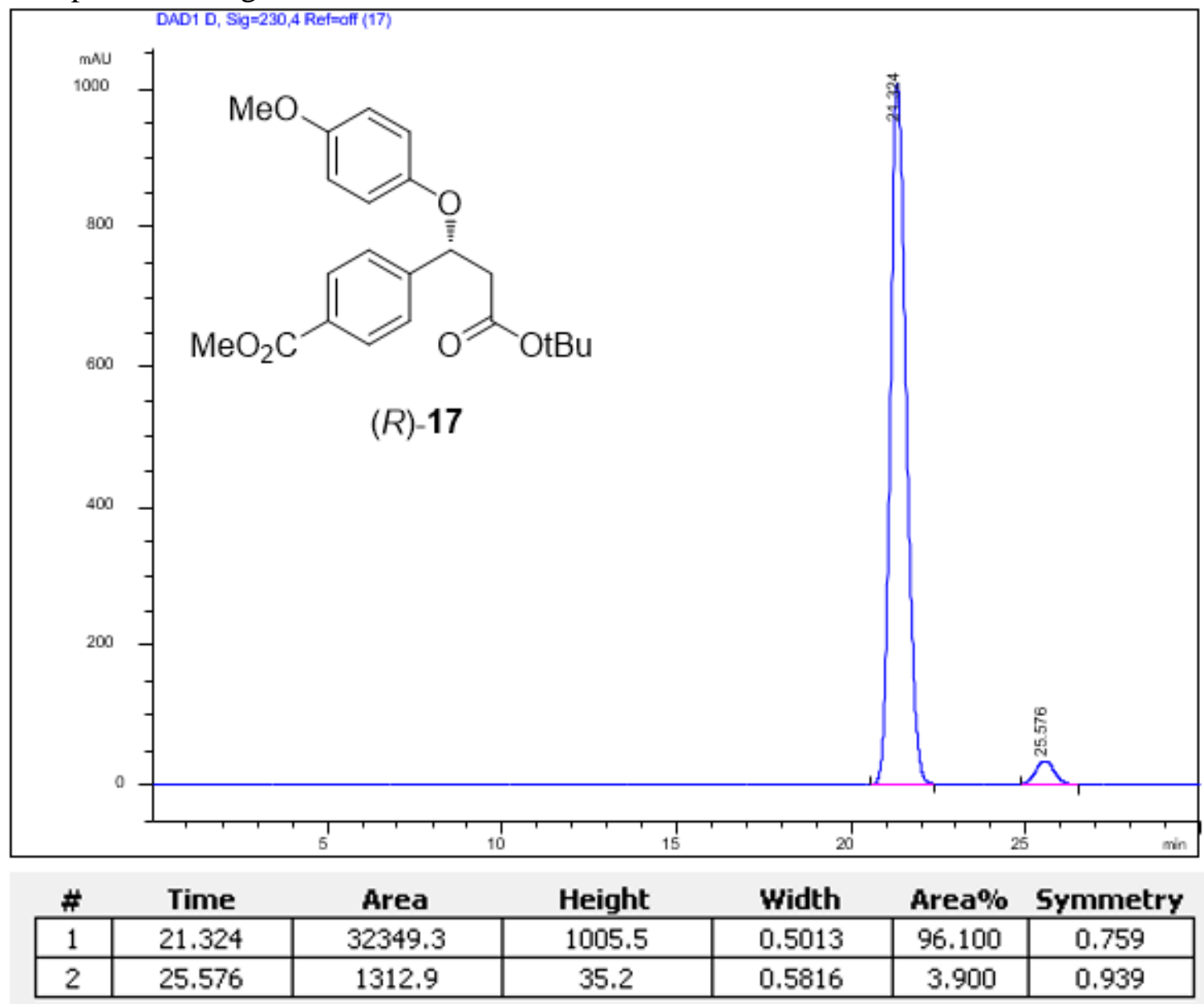


Compound 18, chiral separation:
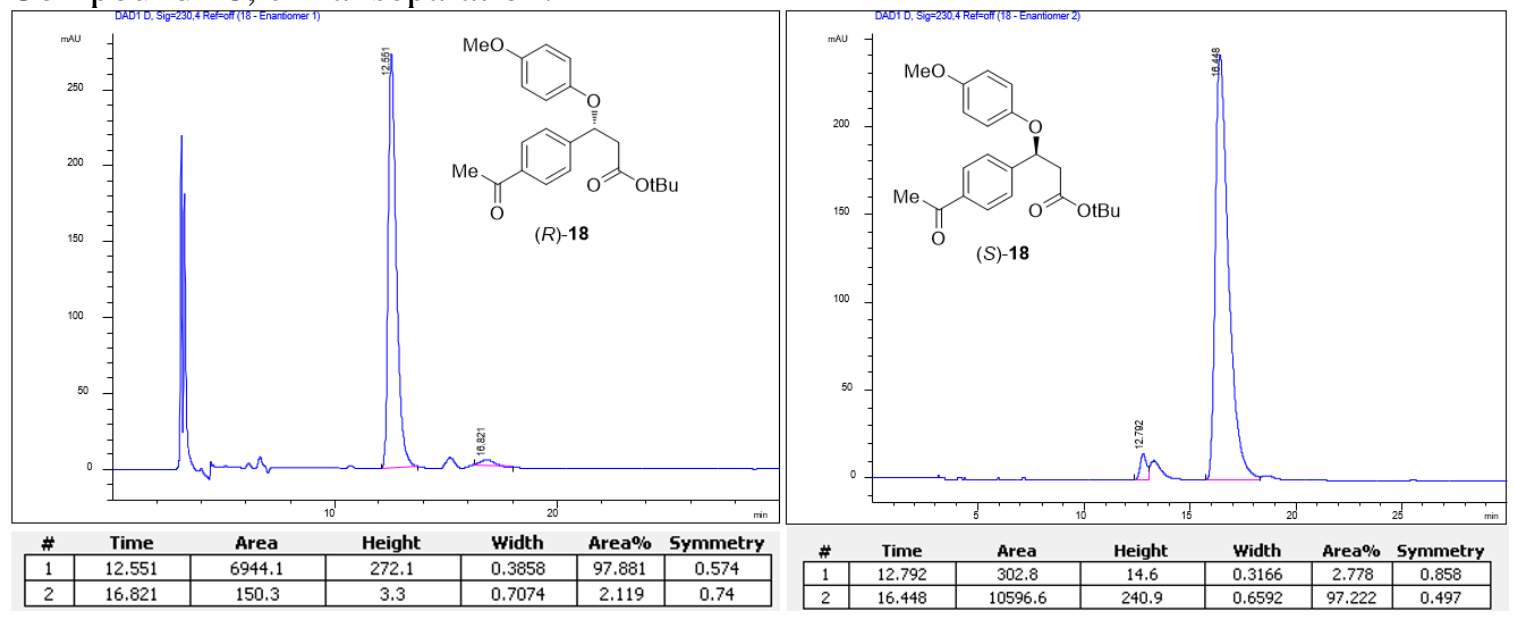

Compound 18, Figure 6:

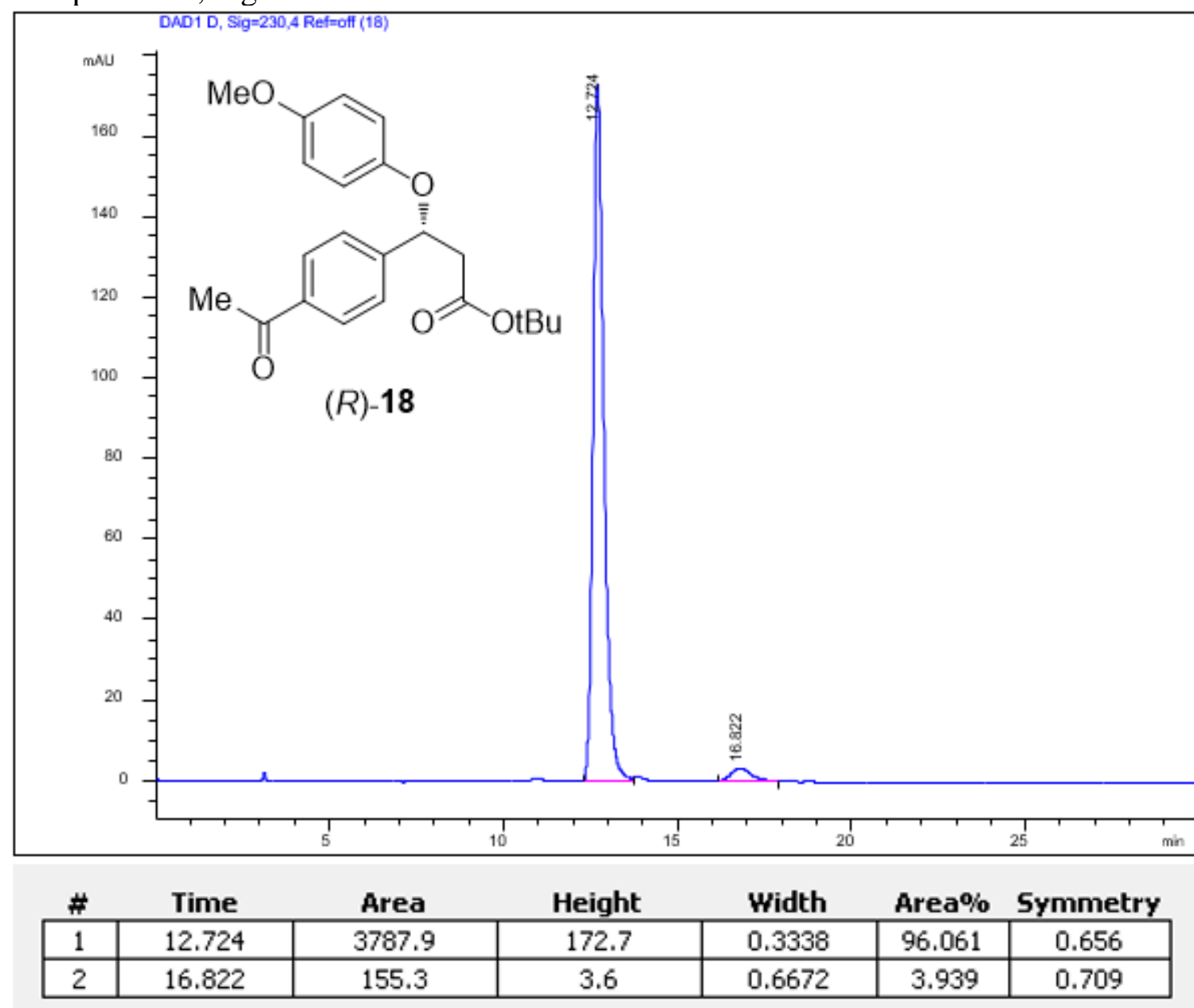


Compound 19, chiral separation:

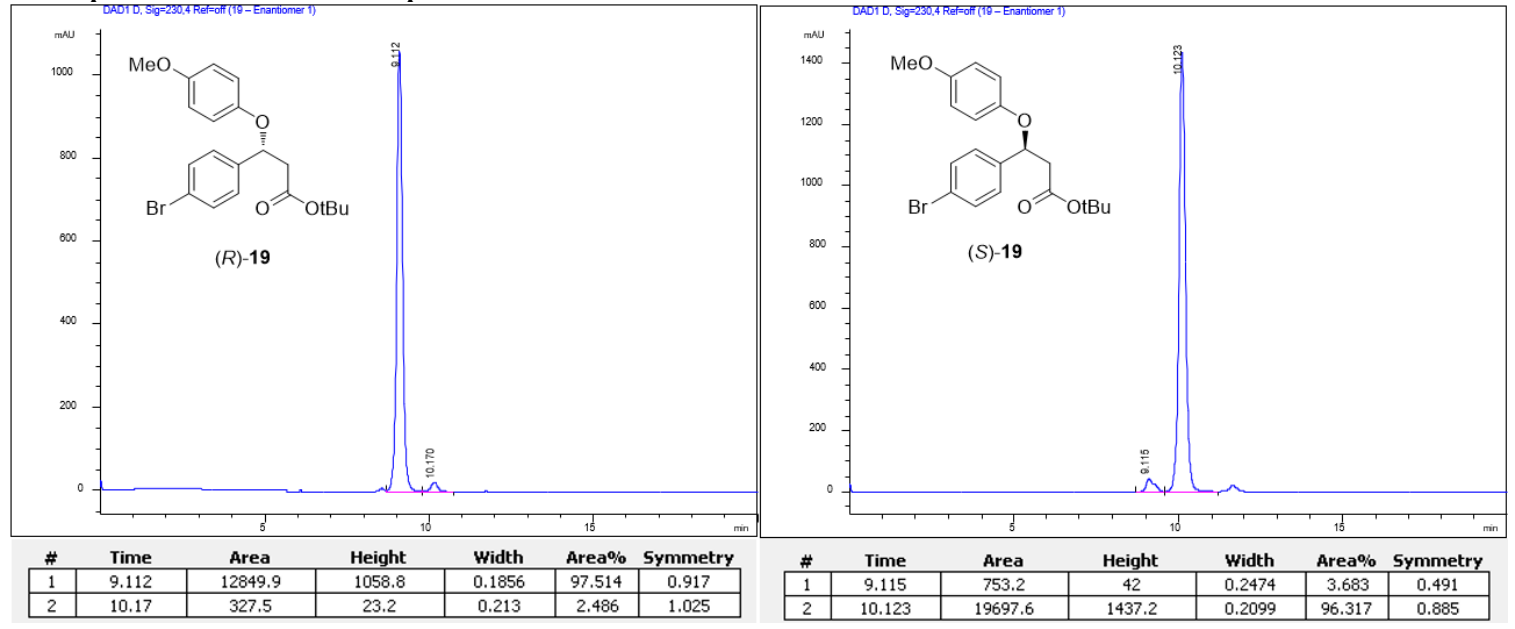

Compound 19, Figure 6:

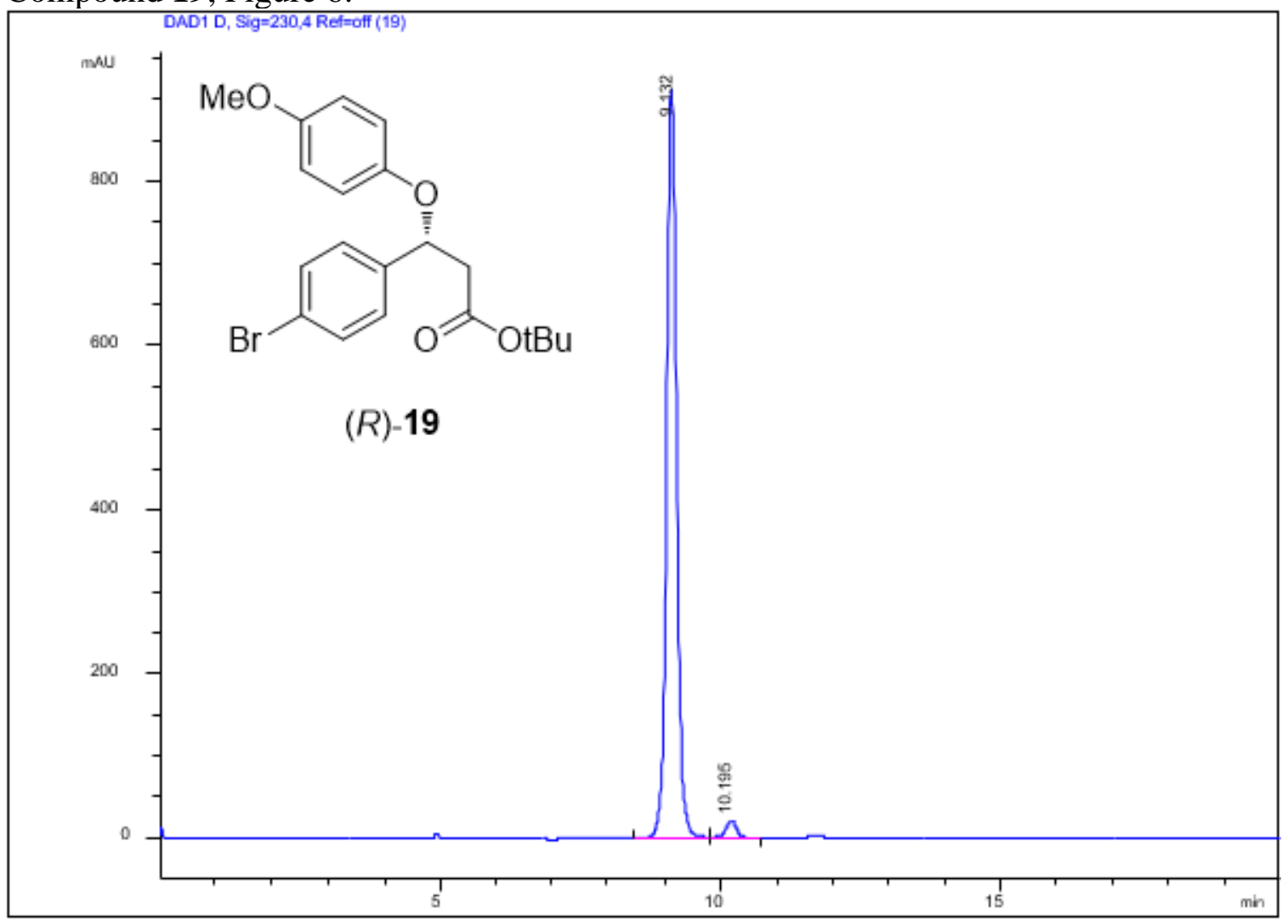

\begin{tabular}{|c|c|c|c|c|c|c|}
\multicolumn{1}{c}{ Time } & \multicolumn{1}{c}{ Area } & \multicolumn{1}{c}{ Height } & \multicolumn{1}{c}{ Width } & \multicolumn{2}{c|}{ Area\% } & Symmetry \\
\hline 1 & 9.132 & 11102.9 & 911 & 0.1862 & 97.379 & 0.918 \\
\hline 2 & 10.195 & 298.9 & 20.9 & 0.2145 & 2.621 & 1.025 \\
\hline
\end{tabular}


Compound 20, chiral separation:
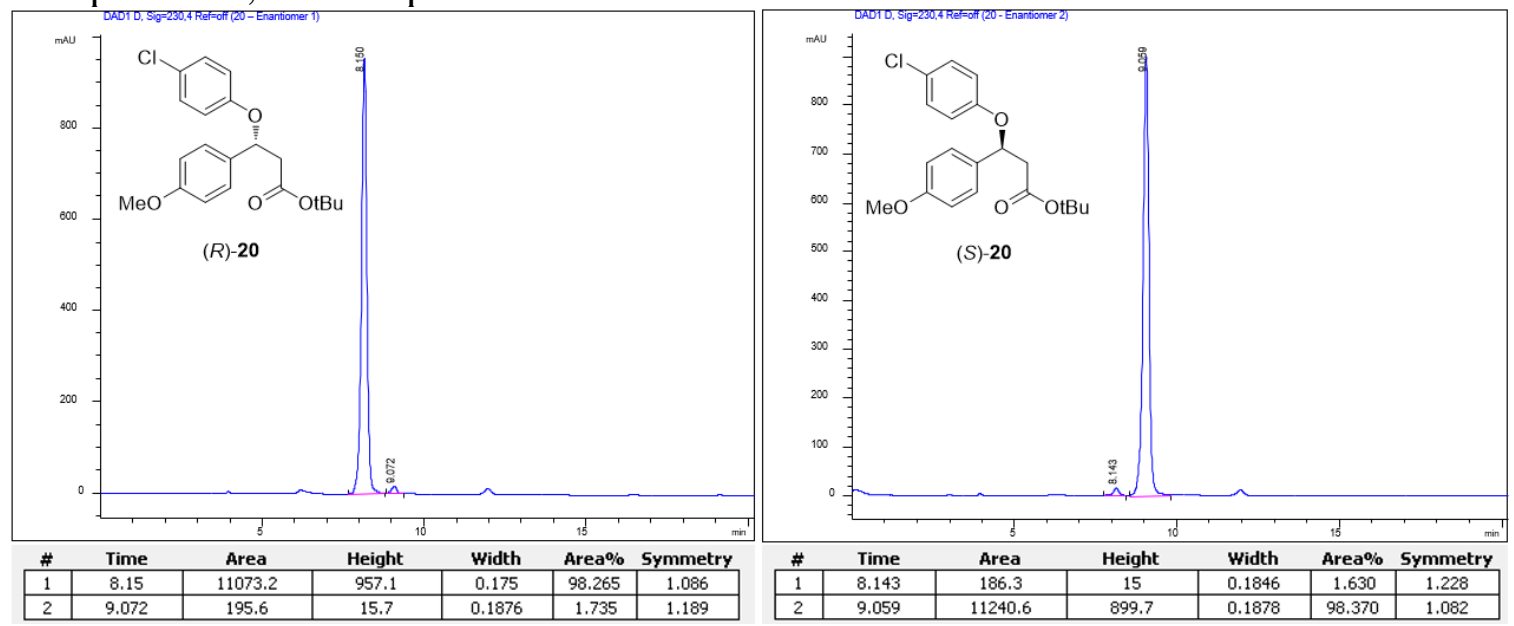

Compound 20, Figure 6:

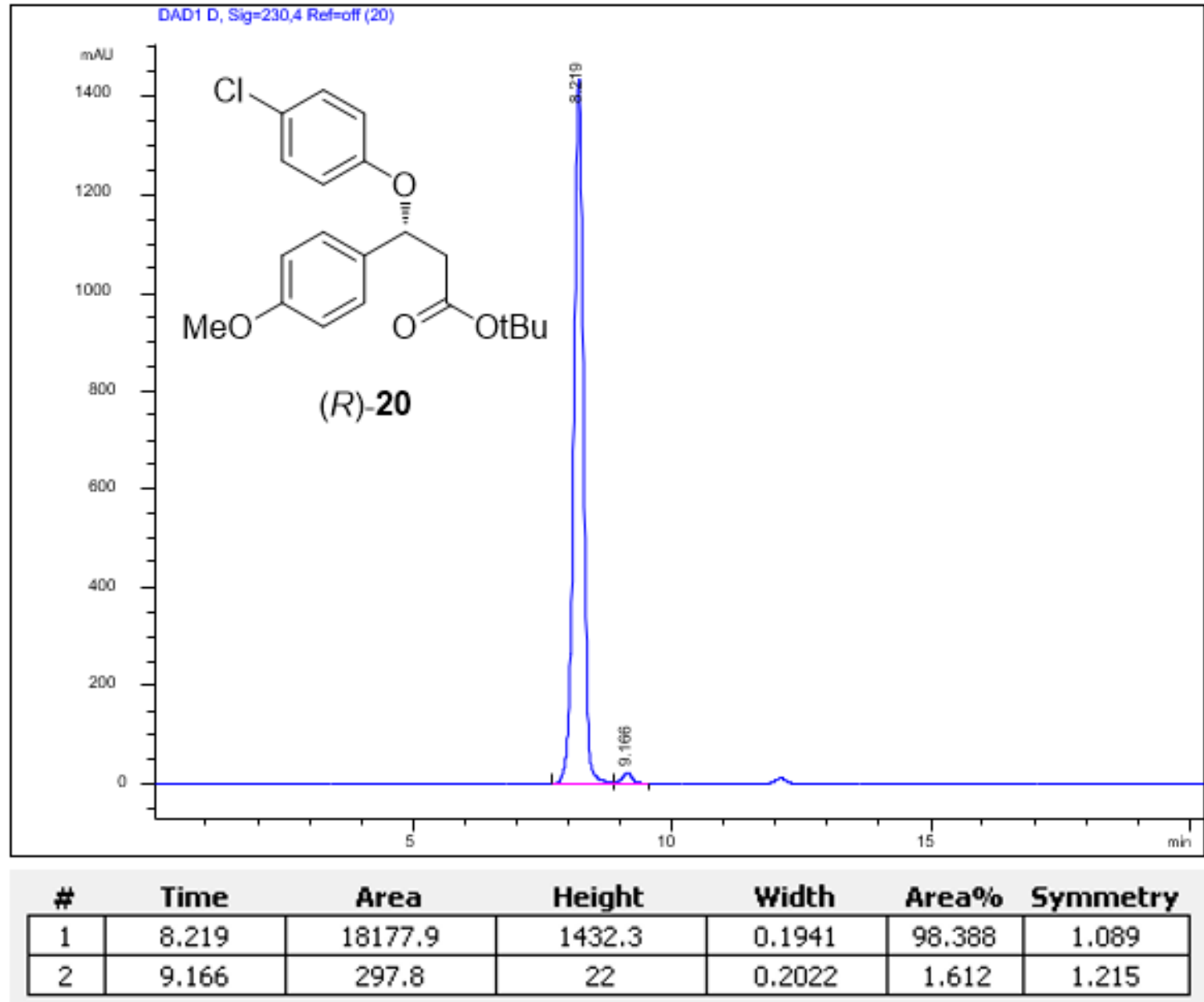


Compound 21, chiral separation:

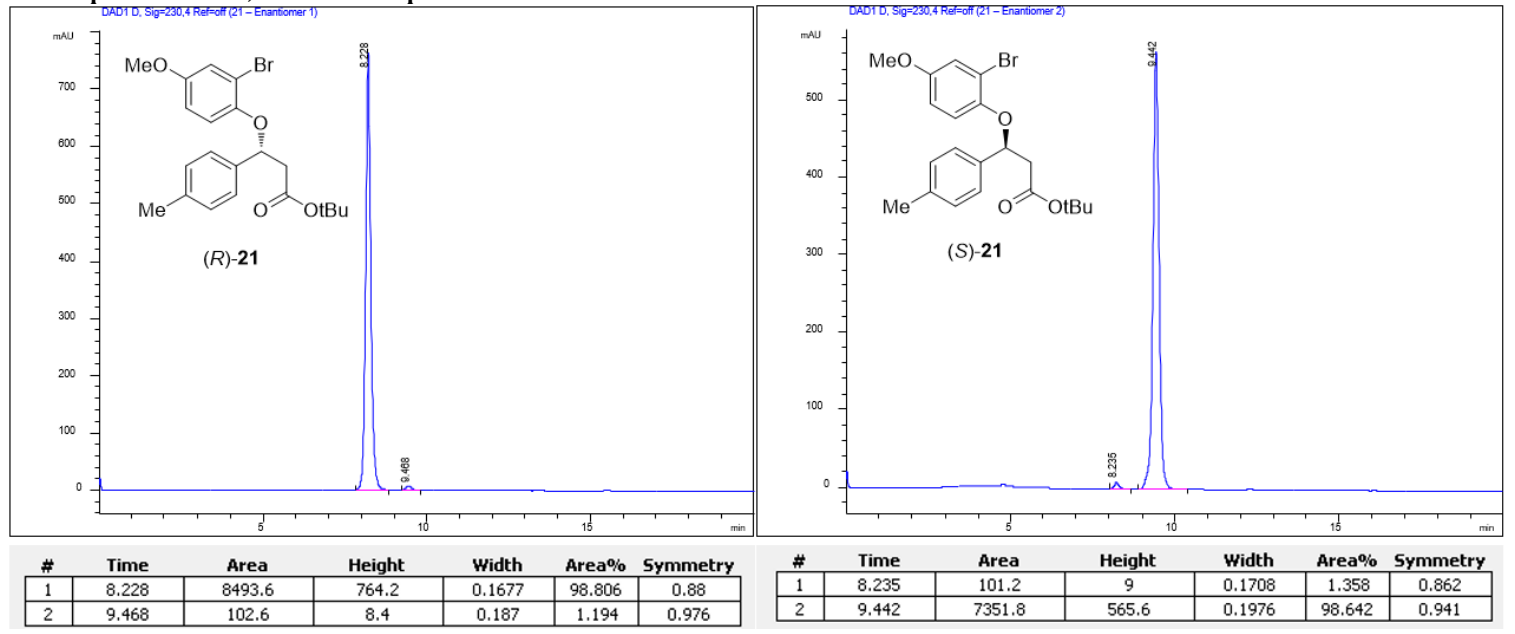

Compound 21, Figure 6:

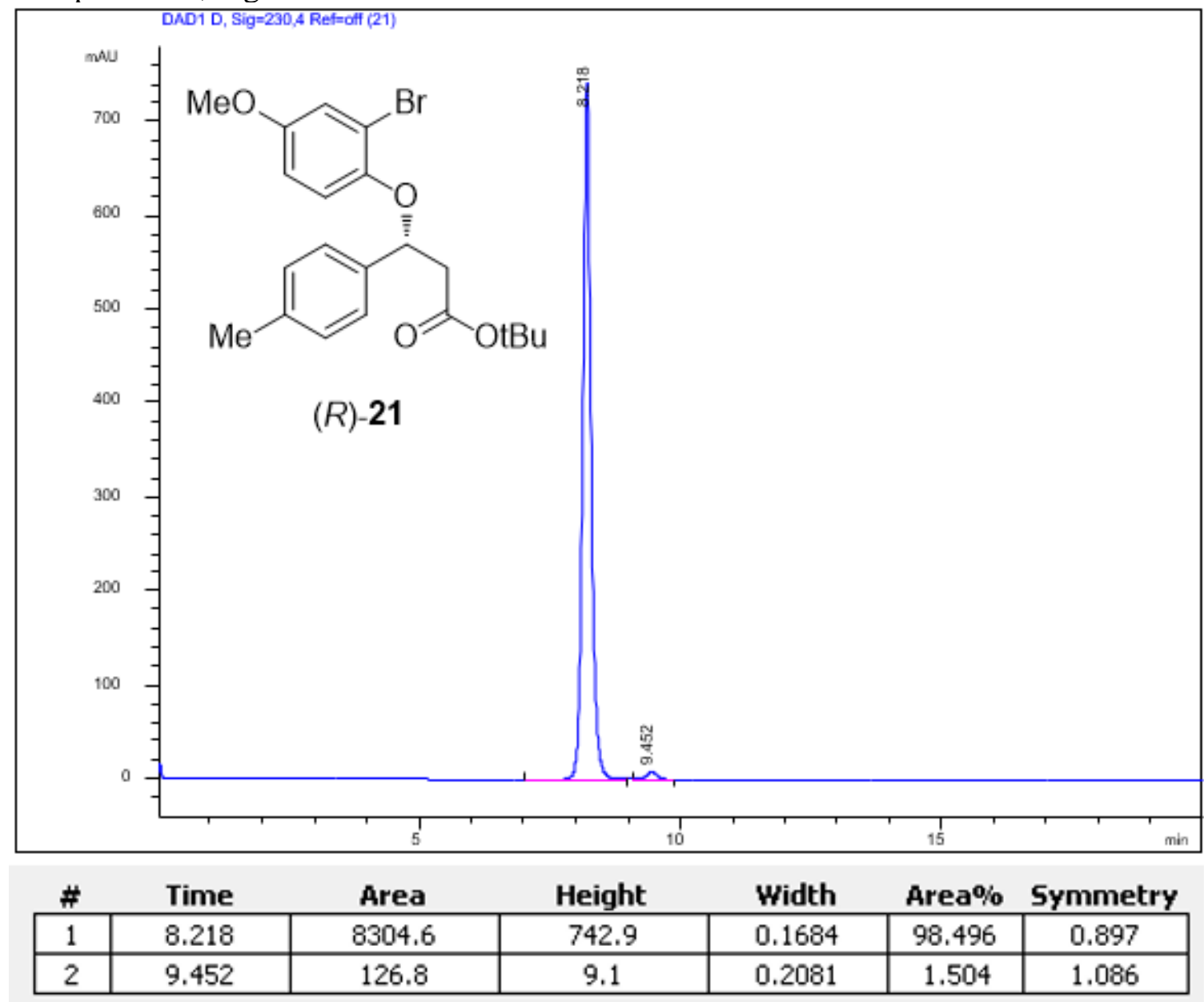


Compound 22, chiral separation:

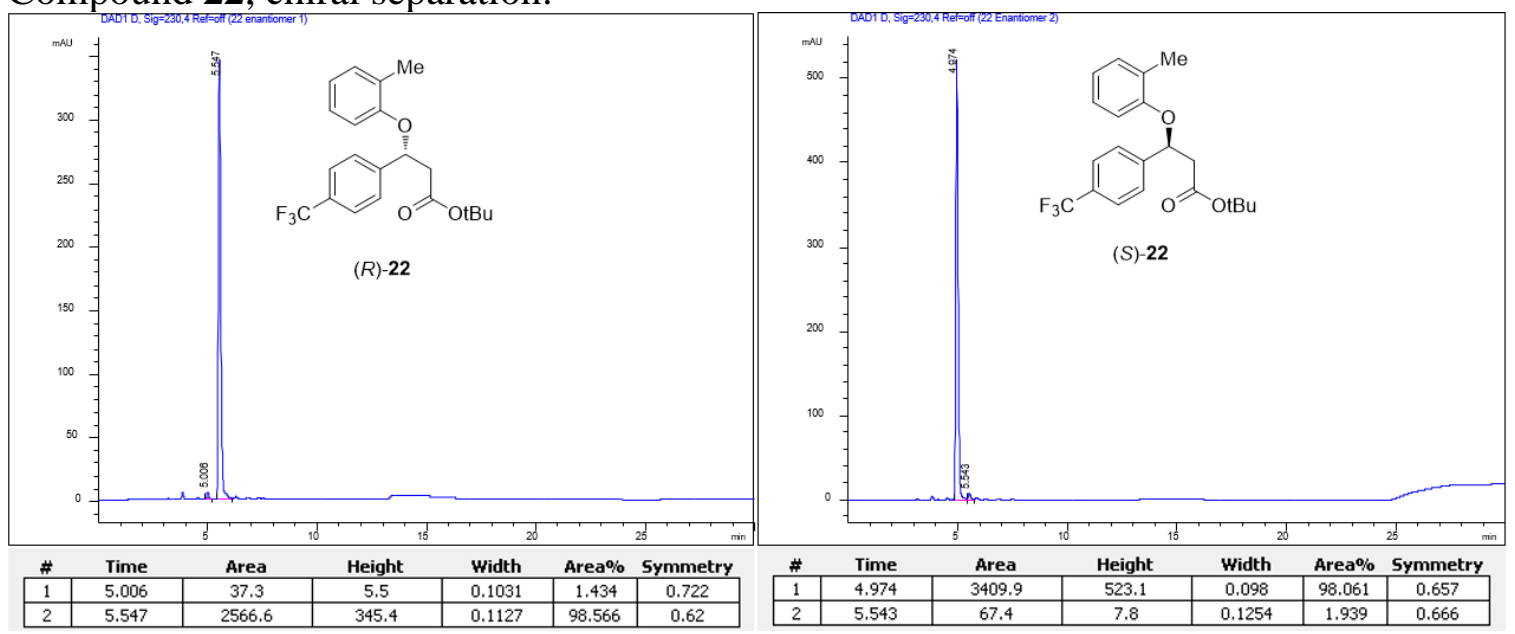

Compound 22, Figure 6:

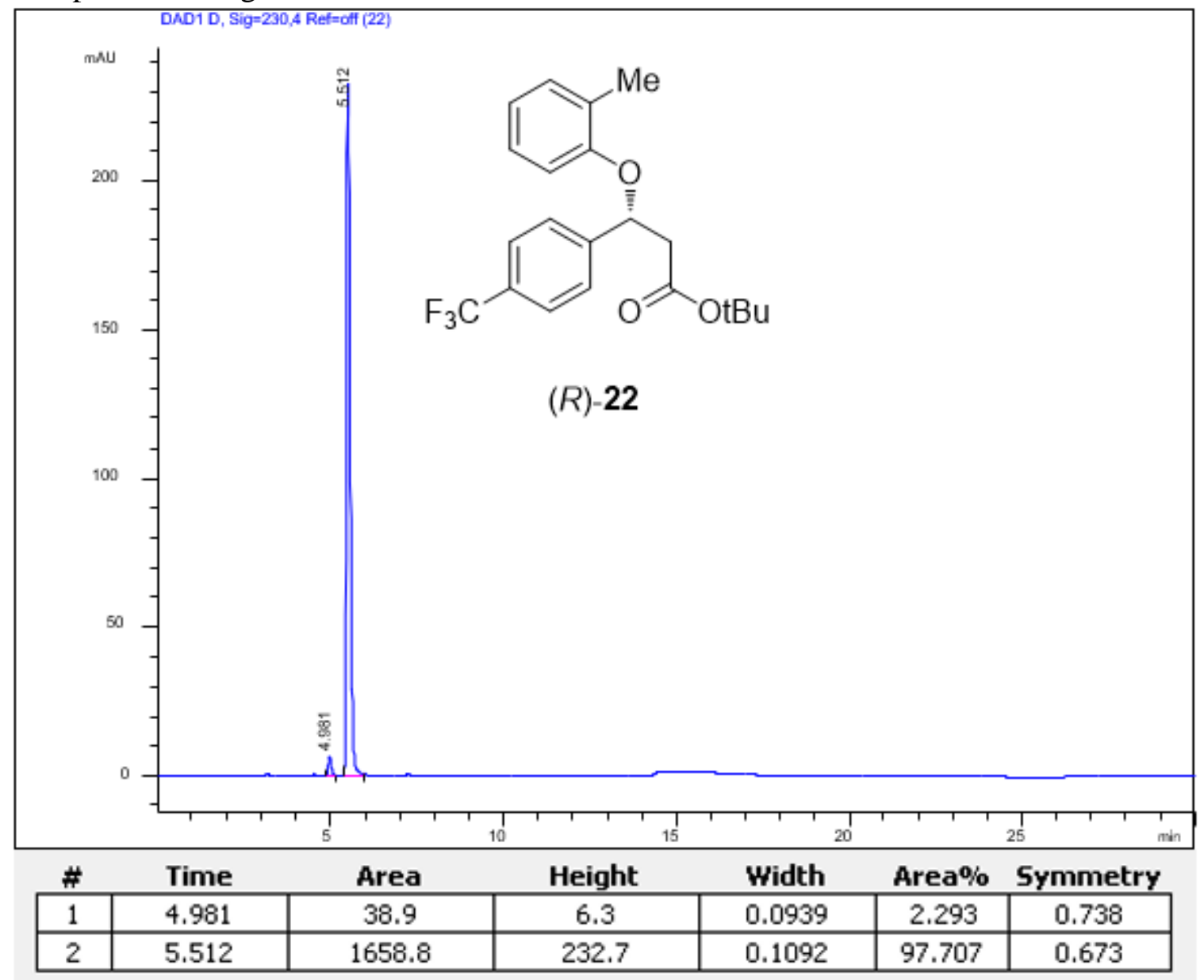


Compound 23, chiral separation:

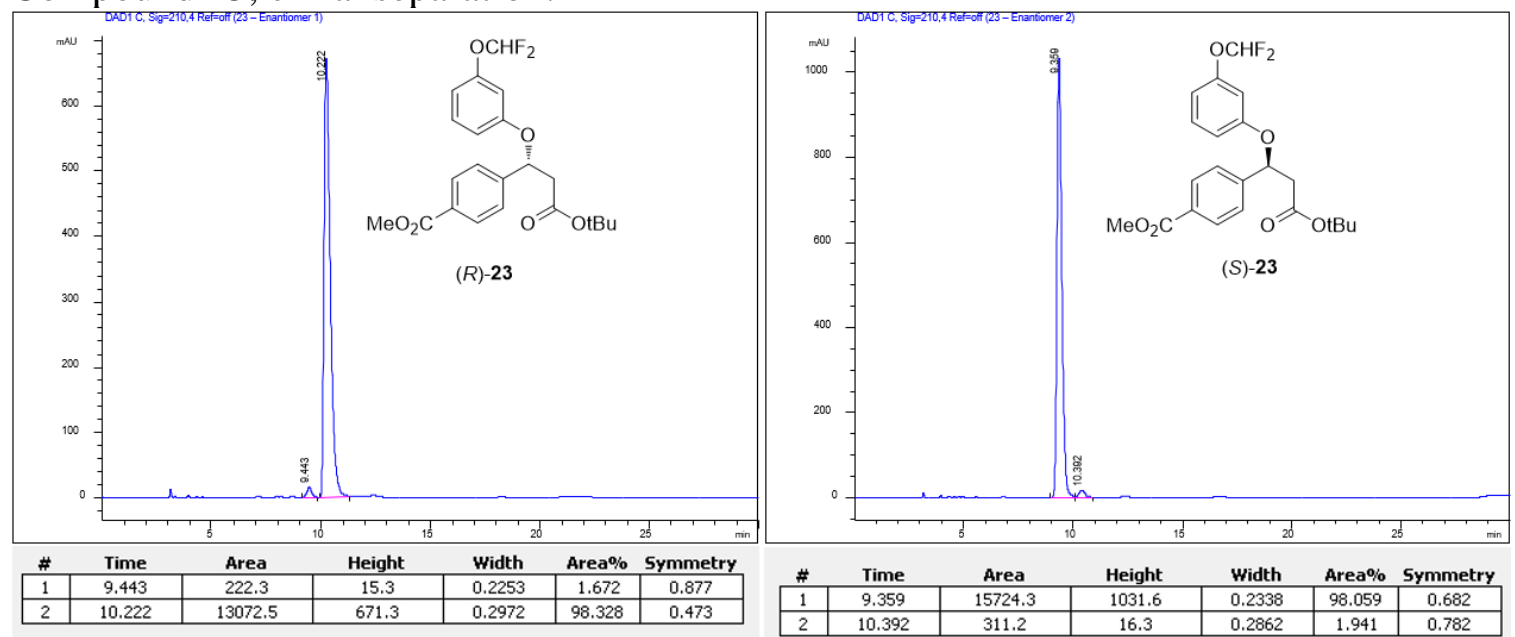

Compound 23, Figure 6:

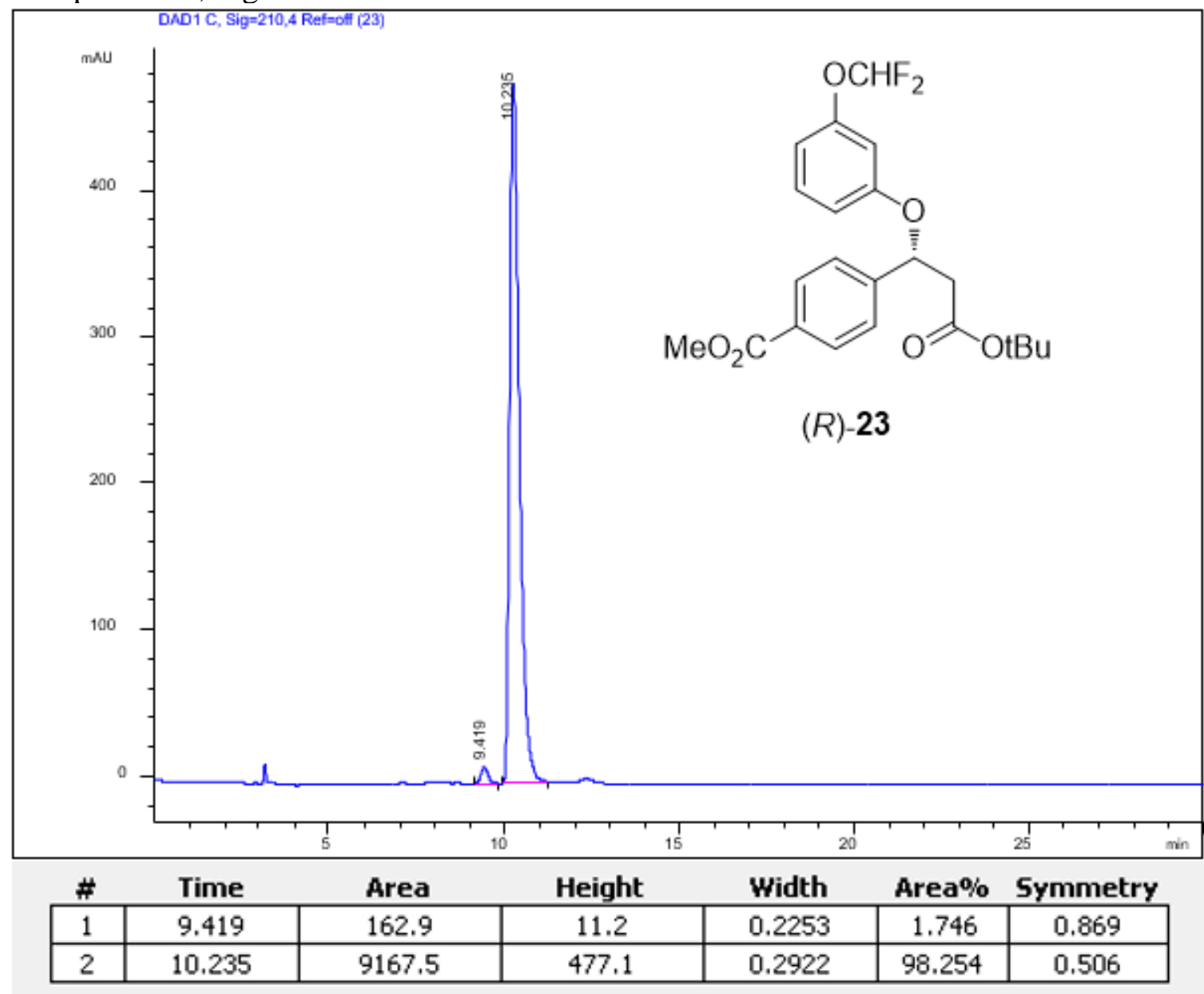

